\title{
EXPLORING THE CONCEPT OF A DEEP SPACE SOLAR-POWERED SMALL SPACECRAFT
}

\author{
A Thesis \\ presented to \\ the Faculty of California Polytechnic State University, \\ San Luis Obispo
}

\author{
In Partial Fulfillment \\ of the Requirements for the Degree \\ Master of Science in Aerospace Engineering
}

by

Kian Crowley

June 2018 
(C) 2018

Kian Crowley

ALL RIGHTS RESERVED 


\section{COMMITTEE MEMBERSHIP}

TITLE:

Exploring the Concept of a Deep Space

Solar-Powered Small Spacecraft

AUTHOR: Kian Crowley

DATE SUBMITTED: June 2018

COMMITTEE CHAIR: Jordi Puig-Suari, Ph.D.

Professor of Aerospace Engineering

COMmitTeE MEMBER: Amelia Greig, Ph.D.

Assistant Professor of Aerospace Engineering

COMMITTEE MEMBER: Kira Abercromby, Ph.D.

Associate Professor of Aerospace Engineering

COMMITTEE MEMBER: Robert Staehle

Jet Propulsion Laboratory 


\begin{abstract}
Exploring the Concept of a Deep Space Solar-Powered Small Spacecraft
\end{abstract}

Kian Crowley

New Horizons, Voyager $1 \& 2$, and Pioneer $10 \& 11$ are the only spacecraft to ever venture past Pluto and provide information about space at those large distances. These spacecraft were very expensive and primarily designed to study planets during gravitational assist maneuvers. They were not designed to explore space past Pluto and their study of this environment is at best a secondary mission. These spacecraft rely on radioisotope thermoelectric generators (RTGs) to provide power, an expensive yet necessary approach to generating sufficient power. With Cubesats graduating to interplanetary capabilities, such as the Mars-bound MarCO spacecraft[1], matching the modest payload requirements to study the outer Solar System (OSS) with the capabilities of low-power nano-satellites may enable much more affordable access to deep space. This paper explores a design concept for a low-cost, small spacecraft, designed to study the OSS and satisfy mission requirements with solar power. The general spacecraft design incorporates a parabolic reflector that acts as both a solar concentrator and a high gain antenna. This paper explores a working design concept for a small spacecraft to operate up to 100 astronomical units (AU) from the sun. Deployable reflector designs, thermal and radiation environments, communications and power requirements, solar system escape trajectory options, and scientific payload requirements are detailed, and a working system is proposed that can fulfill mission requirements with expected near-future innovations in a few key technologies. 


\section{ACKNOWLEDGMENTS}

I'd like to thank several people for helping me with this project. First of all, I'd like to send out a huge thank you to Professor Jordi Puig-Suari and Robert Staehle from JPL for helping me with all the intricacies of this project. They were immensely helpful in every aspect of this paper and always provided excellent advice and suggestions for the design.

I would also like to thank my family and friends for providing the emotional support for getting through this stressful project. Without them, I would have had the motivation to put in the momentous amount of hours into this program. I also would like to thank my peers in this program for providing me with engineering help

on this project, even going to lengths of designing parts of this spacecraft through group class projects. 


\section{TABLE OF CONTENTS}

$\begin{aligned} \text { Page } & \end{aligned}$

LIST OF TABLES . . . . . . . . . . . . . . . . . $\quad$ ix

LIST OF FIGURES . . . . . . . . . . . . . . . . . . . . . xi CHAPTER

1 Introduction . . . . . . . . . . . . . . . . . 1

1.1 Thesis Objectives . . . . . . . . . . . . . . . . . . . 2

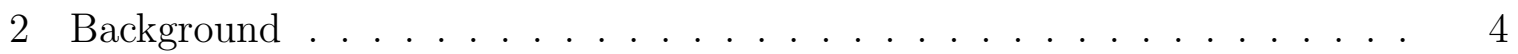

2.1 Past OSS Missions . . . . . . . . . . . . . 4

2.2 Why go to the OSS? . . . . . . . . . . . . 5

2.3 Interstellar Probe Studies f . . . . . . . . . . . . . . . . . 7

2.3.1 1999 NASA Interstellar Probe Study . . . . . . . . . . . 7

2.3.2 Keck Institute for Space Studies (KISS) Interstellar Probe Study 9

2.4 Enabling Technologies . . . . . . . . . . . . . . . 12

2.4.1 Interplanetary CubeSats . . . . . . . . . . . . . . . . 12

2.4.2 Low Power NanoSatellite Electronics . . . . . . . . . . . . . . 13

2.4.3 Small Sat Deployable Technologies . . . . . . . . . . . . 15

3 Light Analysis at $100 \mathrm{AU} \ldots \ldots$. . . . . . . . . . . . . . . . . . .

3.1 Power Generation . . . . . . . . . . . . . . . . . . . . . 18

3.2 Radio Frequency Communication . . . . . . . . . . . . 21

4 System Requirements and Overview . . . . . . . . . . . . . . . . 24

4.1 System Overview . . . . . . . . . . . . . . . 26

4.1.1 Concept of Operations . . . . . . . . . . . . . . 27

4.1.2 Spacecraft Overview ................. 30

5 Orbital Trajectory Options . . . . . . . . . . . . . 36

5.1 Voyager 1 Flyby Sensitivity . . . . . . . . . . . . . . . . . 39

5.2 New Horizons Flyby Sensitivity . . . . . . . . . . . . . . . 42

5.3 Swarm Architecture Possibilities . . . . . . . . . . . . . . . 44

5.4 Viable Orbital Trajectories . . . . . . . . . . . . . . 45

6 Deployable Antenna Subsystem . . . . . . . . . . . . . . . . . . . 47 
6.1 Existing Deployable Technologies . . . . . . . . . . . . . . . . 49

$6.1 .1 \quad \mathrm{Drag} /$ Solar Sails . . . . . . . . . . . . . . . . . . . . . 49

6.1.2 Deployable Reflector Antennas . . . . . . . . . . . . . 51

6.1.3 Inflatable X-Band Antenna (NASA-JPL) . . . . . . . . . . 53

6.2 Antenna Shape Design . . . . . . . . . . . . . . . . 56

6.2.1 Spherical Antenna Alternative . . . . . . . . . . . . . 63

7 Power and Thermal Subsystems . . . . . . . . . . . . . . 67

7.1 Power Subsystem . . . . . . . . . . . . . . . . . . 67

7.1.1 Power Consumption Requirements _. . . . . . . . . 67

7.1.2 High Efficiency Radiation Tolerant Solar Cells . . . . . . . . 70

7.1.3 Power Generation Requirements . . . . . . . . . . . . . . 71

7.1 .4 Battery Sizing . . . . . . . . . . . . . . . 75

7.1.5 Power Subsystem Summary _. . . . . . . . . . . . . 77

7.2 Thermal Subsystem . . . . . . . . . . . . . . . . . . . 78

7.3 Thermal Requirements . . . . . . . . . . . . . . . . 80

7.4 Thermal Desktop Results . . . . . . . . . . . . . . . . . . . . 82

7.4.1 Hot Case Temperature Results . . . . . . . . . . . . 84

7.4.2 Cold Case Temperature Results . . . . . . . . . . . . . 85

7.5 Thermal Subsystem Summary _ . . . . . . . . . . . . 88

7.6 Other Thermal Considerations . . . . . . . . . . . . . . . . . . . . . 89

8 Communications Subsystem . . . . . . . . . . . . . . . . . 9 90

8.1 Link Analysis . . . . . . . . . . . . . . . . . . . 90

8.2 Communications Subsystem Summary _... . . . . . . . 94

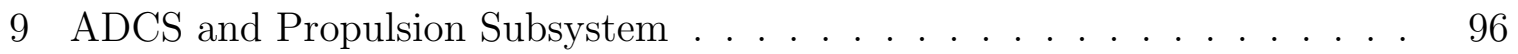

9.1 Attitude Control Requirements . . . . . . . . . . . . . . 97

9.1.1 Calculating Mass Moment of Inertia _. . . . . . . . . . . 99

9.1.2 Calculating Spin Up Impulse Requirement . . . . . . . . . 104

9.2 Actuation Options . . . . . . . . . . . . . . . . . . 105

9.2.1 Photonic Laser Thrusters . . . . . . . . . . . . . . . 105

9.2.2 IKAROS Electro-Chromatic LCD Panels . . . . . . . . . . 106

9.2.3 Traditional Thruster Systems . . . . . . . . . . . . . . . . . . 109

9.3 Thruster Requirements and Trade Study . . . . . . . . . . . . 110 
9.4 Pointing Budget . . . . . . . . . . . . . . . . . 117

9.5 ADCS and Propulsion Subsystem Summary _ . . . . . . . . . 119

10 System Configuration and Configuration Options _. . . . . . . . . 120

10.1 Spacecraft Configuration . . . . . . . . . . . . . . . 120

10.1.1 Deployable Antenna Configuration _. . . . . . . . . 120

10.1.2 Payload Configuration . . . . . . . . . . . . . . 121

10.1.3 Bus Configuration . . . . . . . . . . . . . . 122

10.2 Mass Budget . . . . . . . . . . . . . . . . . . . . . . 124

10.3 Alternative Configuration Options . . . . . . . . . . . . . . 125

11 Radiation Considerations . . . . . . . . . . . . . . . . . . . . . . . 128

12 Conclusion . . . . . . . . . . . . . . . . . . . . . . 131

12.1 Further Work . . . . . . . . . . . . . . . . . . . 132

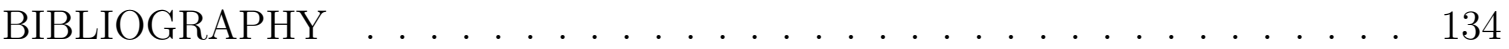




\section{LIST OF TABLES}

Table

Page

2.1 Cost of OSS Spacecraft in FY15 USD [2] . . . . . . . . . . . 4

2.2 Bus Component Standby Power Consumption . . . . . . . . . . . . 14

3.1 Communications Parameters for Deep Space Link . . . . . . . . . 22

3.2 Deep Space Ground Station Options . . . . . . . . . . . . . . 22

4.1 Overall Spacecraft Requirements . . . . . . . . . . . 25

4.2 Science Payload and Data Requirements . . . . . . . . . . . 25

4.3 Power Breakdown for Spacecraft Power Modes at 100 AU . . . . . 29

6.1 Existing Deployable Technologies . . . . . . . . . . . . . . . 49

6.2 Inflatable Antenna Scalability in Size[3] . . . . . . . . . . 56

6.3 Summary of Deployable Parabolic Reflector Parameters . . . . . . . 62

7.1 Power Breakdown for Spacecraft Power Modes at 100 AU . . . . . 68

7.2 Average Power Consumption for Different Operations Cycle Lengths 70

7.3 Solar Concentrator Power Generation Efficiencies . . . . . . . . . . 72

7.4 Average Power Generated as a Function of Concentrator Dish Size . 75

7.5 Major Power Subsystem Characteristics . . . . . . . . . . 78

7.6 Component Level Thermal Requirements . . . . . . . . . . . . . . . 81

7.7 External Optical Properties for Bus and Radiator Panels[4] . . . . 83

7.8 Maximum and Minimum Temperatures of Components at the Hot Case ...................... 86

7.9 Maximum and Minimum Temperatures of Components at the Cold Case ........................... 88

7.10 Thermal Subsystem Summary . . . . . . . . . . . . 88

8.1 Communications Parameters for Deep Space Link . . . . . . . . . 91

8.2 Communications Subsystem Characteristics Summary . . . . . . . 95

9.1 Deployed Spacecraft Components and Geometric Approximations . 102 
9.2 Deployed Spacecraft Mass Moment of Inertias . . . . . . . . . . . . 102

9.3 Undeployed Spacecraft Components and Geometric Approximations 103

9.4 Undeployed Spacecraft Mass Moment of Inertias and CM . . . . . . 103

9.5 Impulse Requirements for All ADCS and Propulsion Maneuvers . . 111

9.6 Busek BGT-X1 100mN Green Propellant Thruster Parameters . . . 113

9.7 Spacecraft Pointing Budget . . . . . . . . . . . . . 118

9.8 Summary of ADCS and Propulsion Subsystem Characteristics . . . 119

10.1 Required Bus Electronics and Dimensions . . . . . . . . . . . 123

10.2 Spacecraft Master Mass Budget . . . . . . . . . . . . . 125 


\section{LIST OF FIGURES}

Figure

2.1 Interstellar Medium Interaction[5] . . . . . . . . . . 6

2.2 1999 NASA Interstellar Probe Trajectory and Solar Sail Maneuvers[6] 9

2.3 KISS Interstellar Probe Trajectory Solutions; a) Solutions using Hall Thruster, b) Solutions using E-sails [7] . . . . . . . . . . . . . . . . 11

3.1 Power Received at Various Stages During Power Generation Processes 20

3.2 RF Power Required vs. Reflector Dish Size at 100 AU . . . . . . 23

4.1 Overall Block Diagram of Spacecraft Subsystems . . . . . . . . . 27

4.2 Spacecraft Without Deployed Mechanisms . . . . . . . . . . . 31

4.3 Spacecraft With Fully Deployed Inflatable Antenna, Radiator Panels, and Payload Booms . . . . . . . . . . . . . . 32

4.4 Deployable Radiator Deployment Sequence . . . . . . . . . . . . 34

4.5 Payload Boom Deployment Sequence . . . . . . . . . . . . . 35

5.1 Trajectory Effects of Small Delta-V Maneuver Prior to Saturn Flyby 40

5.2 Visual of Trajectories as a Result of Saturn Flyby Sensitivity Analysis 41

5.3 Trajectory Effects of Small Delta-V Maneuver Prior to Jupiter Flyby 43

5.4 Visual of Trajectories as a Result of Jupiter Flyby Sensitivity Analysis 44

6.1 Deployable Reflector Concept Showing Power Generation and HighGain Antenna Functions . . . . . . . . . . . . . . . . . . 48

6.2 Packing Efficiency Trending of Solar and Drag Sails . . . . . . . 51

6.3 Packing Efficiency Trending of Deployable Antenna Systems . . . . 53

6.4 NASA JPL Inflatable Antenna in an Anechoic Chamber[8] . . . . . 55

6.5 Parabola Parameters . . . . . . . . . . . . . . . 57

6.6 The Focal Point Problem _. . . . . . . . . . . . . . . . 58

6.7 Efficiency Loss as a Function of Axial Displacement of the Focal Point from the Feed, courtesy of Paul Wade, W1GHZ[9] . . . . . . 59

6.8 Focal Area from Parabolic Concentration for Various Solar Panel Width Cases and Optimal Focal Area . . . . . . . . . . . . . . . . 60 
6.9 Axial Displacement in X-Band Wavelengths as a Function of Solar Panel Widths . . . . . . . . . . . . . . . . . . . . 61

6.10 Resulting Parabolic Dish Shape . . . . . . . . . . . . 63

6.11 Spherical Dish Ray Tracing for Nominal and 5 Degree Off Axis Case by Dr. Howard Haber[10] . . . . . . . . . . . . . . . . . . . . . . . 64

6.12 Parabolic Dish Ray Tracing for Nominal and 5 Degree Off Axis Case by Dr. Howard Haber[10] . . . . . . . . . . . . . . . . . . 65

7.1 Effective Sunlit Area on Solar Panel and Feed Face . . . . . . . . 74

7.2 Thermal Desktop Results for the Hot Case at Jupiter . . . . . . . . 85

7.3 Thermal Desktop Results for the Cold Case at 100 AU . . . . . . . 87

8.1 RF Power Required for a $7.75 \mathrm{~m}$ Diameter Dish at 10 bps at Various Distances From the Sun . . . . . . . . . . . . . . . 93

9.1 Attitude Angle Change During Voyager 1 Trajectory From Jupiter to $100 \mathrm{AU}$. . . . . . . . . . . . . . . . 98

9.2 Attitude Rates During Voyager 1 Trajectory From Jupiter to 100 AU 98

9.3 Spacecraft Configuration Geometry Approximations for Deployed and Undeployed State . . . . . . . . . . . . . . . 100

9.4 Spacecraft Configuration Relative Positions . . . . . . . . . . . 101

9.5 IKAROS Attitude Control LCD Panels[11] . . . . . . . . . . . . 107

9.6 Total Impulse Required for Tracking Earth Along Voyager 1 Trajectory as a Function of Moment Arm and Spin Rate . . . . . . . . . . 110

9.7 Thruster Trade Study . . . . . . . . . . . . . . . . . . 112

9.8 Possible Thruster Configuration in Bus . . . . . . . . . . . . . . . . 114

9.9 a) Number of Required Attitude Correction Maneuvers Using S-Band Beamwidth; b) Frequency of Maneuvers . . . . . . . . . . . . . 115

9.10 a) Number of Required Attitude Correction Maneuvers Using XBand Beamwidth; b) Frequency of Maneuvers . . . . . . . . . . . . 116

9.11 Spin Axis Angle Change for $14 \mathrm{mNs}$ Minimum Impulse Bit as a Function of Moment Arm and Spin Rate . . . . . . . . . . . . 118

10.1 Inflatable Antenna Configuration When Packaged . . . . . . . . . . 121

10.2 Component Configuration of Bus . . . . . . . . . . . . . 123

10.3 Tendeg's Off-Center Fed KaTENna High Gain Cubesat Antenna[12] 126

10.4 Alternative Configuration Using Boom Deployed Parabolic Dish . . 127 
11.1 Radiation Environments in Various Regions of the Solar System[13] 129 


\section{Chapter 1}

\section{INTRODUCTION}

The Voyager spacecraft represent a portion of the very few data points we have from the Outer Solar System (OSS), leaving us with little knowledge of the environment at those distances from the Sun. While both Voyager 1 and 2 have crossed the region of termination shock, with Voyager 1 also crossing the heliopause, these remain the only in-situ data of these regions. Furthermore, despite the Voyager spacecraft visiting these deep space locations, they crossed outside of the ecliptic plane, with Voyager 1 heading south after the Saturn flyby and Voyager 2 heading north after its encounter with Neptune[14]. There has been some recent work challenging the current best model of the heliopause, however, with little in-situ data, these suggestions are unable to be confirmed. This leaves the OSS largely unexplored and beckoning for further study with additional missions. These dedicated OSS missions are infrequent in part because of the high cost of radioisotope power systems. Low solar intensities at large distances from the sun often require deep space probes to incorporate these expensive nuclear power-sources. As a result, the only missions that have explored this region of space are planetary fly-by spacecraft, some of which are now on extended secondary

missions. A non-nuclear power solution would reduce cost significantly and enable more affordable access to deep space. The moderate sensor requirements for OSS missions coupled with the low power requirements of the latest generation of small satellite avionics can result in spacecraft with minimal power requirements when compared with traditional spacecraft. These reduced power requirements, as well as new developments in small satellite deployable technologies, make solar power and small mass an option for these spacecraft.

Small deep space spacecraft using solar power offer benefits in terms of trajectory 
solutions and scientific return. Employing a rideshare system with primary OSS spacecraft to place these smaller spacecraft on solar system escape trajectories would allow a single mission to visit two deep space destinations, multiplying the scientific yield of a mission while adding only minimal cost. On the other hand, a primary OSS mission can be designed that incorporates a host spacecraft carrying 5 or 10 small spacecraft. The carrier spacecraft would dispense each of the smaller spacecraft at the appropriate times during or before a gravitational assist, dispersing these various spacecraft across a large swath of deep space. Exploring the concept of a spacecraft that can operate with the use of solar power at distances of up to $100 \mathrm{AU}$ can result in a more affordable platform for returning scientific measurements from deep space. Avoiding the use of nuclear power sources will not only reduce spacecraft cost, but reduce rideshare and launch-related concerns and tedious paperwork.

\subsection{Thesis Objectives}

This paper aims to analyze requirements and ultimately design a working spacecraft concept that satisfies mission requirements. The main thesis objectives are stated below.

- Design a working spacecraft concept.

- Spacecraft must operate and support payload at a maximum of 100 AU.

- Spacecraft must operate using solar power.

- Spacecraft must return a sufficient amount useful data.

- Spacecraft must be low mass and small dimensions.

The objectives of this thesis should culminate in a spacecraft design concept that is small, low-cost, and able to return data of the deep space environment. Along the 
way, the paper also provides a wide overview of all the requirements and considerations when designing a small solar-powered OSS probe. It lays down the groundwork for designing a type of probe that, otherwise, has been rarely investigated. 
Chapter 2

\section{BACKGROUND}

\subsection{Past OSS Missions}

Deep space spacecraft have come far and few between. Pioneer 10 and 11, Voyager 1 and 2, and New Horizons remain the only spacecraft to have ventured past Pluto and returned scientific data at those distances. These flagship NASA missions, although primarily designed for a variety of planetary flybys, can cost on the order of hundreds of millions, even billions, of dollars. Table 2.1 shows the costs of these OSS spacecraft in FY15 USD to adjust for inflation.

Table 2.1: Cost of OSS Spacecraft in FY15 USD[2]

\begin{tabular}{ccc}
\hline Spacecraft & Pre-Adjusted Cost (USD) & FY15 Cost (USD) \\
\hline Voyagers (each; averaged) & $\$ 865,000,000(1977)$ & $\$ 3,600,000,000$ \\
New Horizons & $\$ 700,000,000(2006)$ & $\$ 900,000,000$ \\
Pioneer 11 & $\$ 150,000,000(1972)$ & $\$ 850,000,000$ \\
\hline
\end{tabular}

Although Pioneer 10 has crossed the termination shock region, it has been unable to communicate with Earth for the last decade and a half, returning its last signal at distances of about $82 \mathrm{AU}$ in 2003. Pioneer 11 has also crossed this region although ceased communicating with Earth, in 1995, nearly a decade before Pioneer 10[15]. With New Horizons currently at about 40 AU[16], the only spacecraft returning data past this region of termination shock are the two Voyager spacecraft. However, the Voyager spacecraft are expected to operate until around 2025, leaving the New Horizons spacecraft as the only remaining spacecraft capable of reaching these distances and returning data after the Voyager spacecraft shut down, assuming it will survive the long journey. This leaves us with only two instances of active spacecraft crossing 
termination shock and currently one instance of crossing into the interstellar medium, possibly soon two instances when Voyager 2 reaches the same distance as the Voyager 1 interstellar medium crossing in 2019/2020. Furthermore, Voyager 1 had been operating without its plasma spectrometer since 2007 due to degrading performance, leaving the magnetometer data without the crucial pairing of plasma environment data soon after it crossed the termination shock zone[17]. The New Horizons spacecraft is also lacking a magnetometer due to budget and time constraints, requiring the need to infer such data through other means. Although interstellar probes are constantly investigated, there are no future missions planned to visit deep space past Saturn[18].

\subsection{Why go to the OSS?}

As explained in the previous section, there are only two spacecraft that have provided information at distances greater than $100 \mathrm{AU}$, with a third planned to reach those distances in at least a couple decades. Even with New Horizons, this leaves the scientific community with little data about the OSS environment. Although there are models of this deep space environment, there is still much to be known about this vast area. What is specifically interesting is the interaction between the solar wind and the interstellar medium around our solar system. In 1955, Leverett Davis proposed that the "solar corpuscular radiation" would result in a cavity in our local galactic magnetic field, and he proposed it was roughly $200 \mathrm{AU}$ in radius[14]. After this, interest in this region increased and the thought of a deep space probe emerged to record in-situ data of this interaction between solar wind and the interstellar medium. This would later become the Pioneer 10 mission, although several mission goals did change. Although current modeling suggests a "windsock" model for the cavity, known as the heliopause, Cassini and Voyager data have challenged these models and 
shown a more bubble-like shape, as suggested by Davis[14]. The interaction between the solar system and the interstellar medium are best described in shells. Figure 2.1 shows a graphic of this interaction and its various layers.

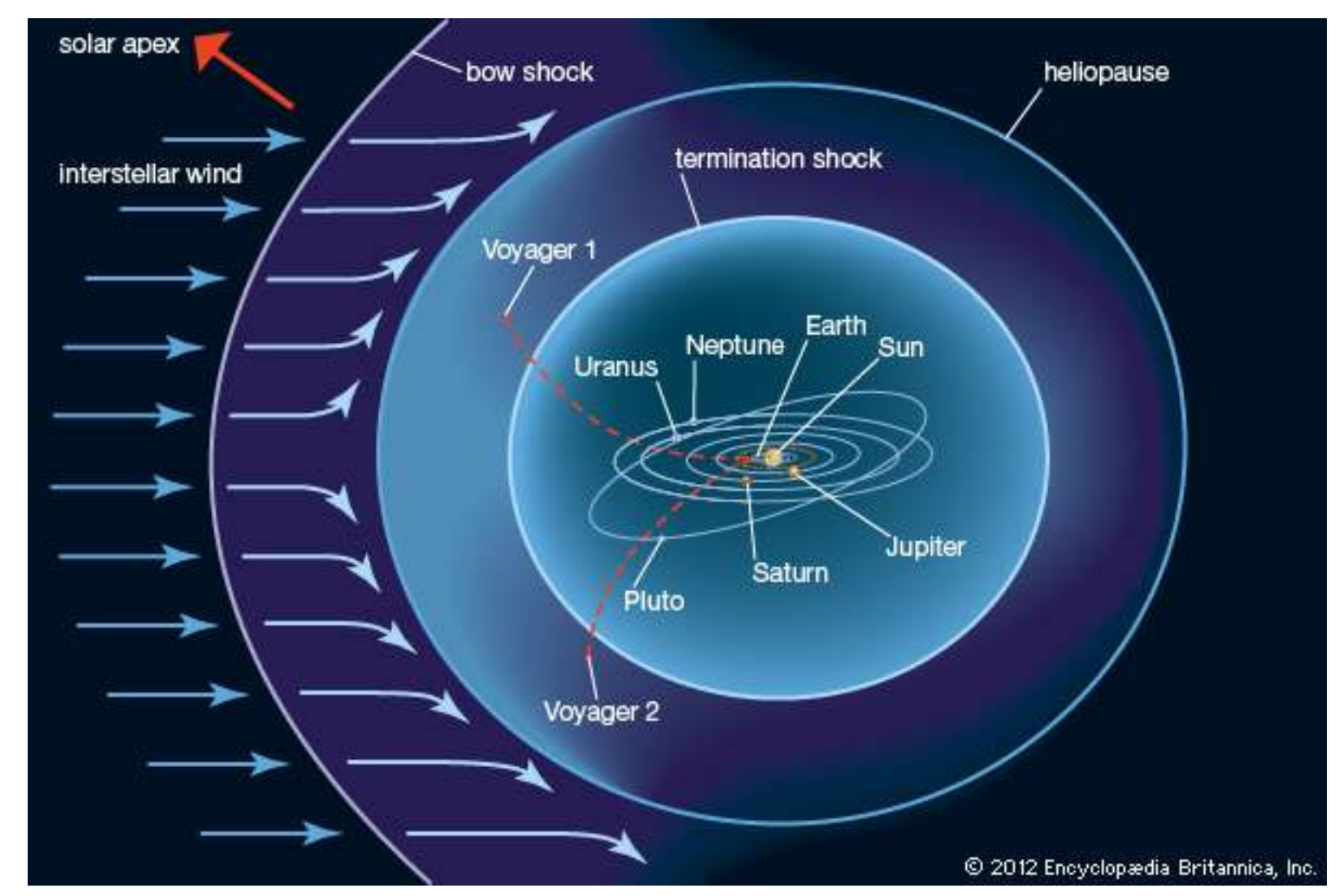

Figure 2.1: Interstellar Medium Interaction[5]

Supersonic solar wind is constantly ejected from the Sun's corona, permeating through the solar system. As the solar system moves through interstellar space, this solar wind pushes against the interstellar medium and creates a pressure between the two mediums. This results in a bow wave or a bow shock that resembles a boat moving through water. The solar wind that is radially radiating out from the sun pushes the interstellar medium around it, leaving a wake zone opposite of the solar system's velocity. This solar wind drastically slows down at the first layer of interaction known as the termination shock region. This region is followed by the heliosheath, which describes the region between termination shock and the heliopause where the solar 
wind starts to flow back around the solar system. After the heliosheath comes the heliopause, where the solar wind and the interstellar medium pressures are at an equilibrium. The final interaction zone is called bow shock where the interstellar medium begins to slow down as it begins to feel the pressure from the solar wind. [19] The Voyager missions have indeed crossed regions up through the heliopause, however, these two data points still leave much to be known. Added data points from in-situ measurements at various locations would help better understand the shape of those various interstellar regions.

\subsection{Interstellar Probe Studies}

The idea of an interstellar probe has been studied since at least the 1950s. Many early studies examined large spacecraft propelled with highly expensive and undeveloped nuclear propulsion technologies. Project Orion was a manned spacecraft examined in 1958 that would detonate nuclear fission bombs behind the spacecraft to provide meganewtons of thrust and propel the spacecraft to high speeds. This idea was not pursued due to possible contamination of near-Earth space with fallout, the large costs and amount of mass required to create the spacecraft, and, ultimately, the passing of the Partial Test Ban Treaty in 1963, which banned nuclear explosives tests in space[20]. However, since then, more feasible designs have been studied for unmanned interstellar probes.

\subsubsection{NASA Interstellar Probe Study}

NASA conducted a study in 1999 to investigate possible interstellar probe and mission designs to reach $200 \mathrm{AU}$ in less than 15 years[6]. The spacecraft design used technology consistent with expected developments by 2007. The mission would launch in 2010 and reach 200 AU distances 15 years later. The probe would carry a relatively 
substantial payload of roughly 12 instruments to study the deep space environment throughout its mission. The final proposed design included a 410 meter diameter circular solar sail with an areal density of $1 \mathrm{~g} / \mathrm{m}^{2}$. The spacecraft was roughly $600 \mathrm{~kg}$ with a payload mass of $25 \mathrm{~kg}$. It used advanced radioisotope thermoelectric generators (RTGs) for power generation and a rigid $2.7 \mathrm{~m}$ high gain parabolic dish antenna for communications back to Earth.The design also incorporated 10 radioisotope heater units to keep the spacecraft warm. The team estimated costs to amount to less than $\$ 500$ million.

In order to achieve solar system escape velocities, the spacecraft would drop to a perihelion distance of $0.25 \mathrm{AU}$ after launch. After this maneuver, the spacecraft would propel itself to high velocities by orienting its solar sail towards the sun. The high solar intensities would provide the spacecraft with considerable acceleration, ultimately receiving approximately $70 \mathrm{~km} / \mathrm{s}$ of delta- $\mathrm{V}$ by the time it reaches $5 \mathrm{AU}$. Figure 2.2 shows the trajectory and attitude maneuvers of the interstellar probe. The spacecraft will then jettison its solar sail at $5 \mathrm{AU}$ and continue its mission to $200 \mathrm{AU}$ at a velocity of just under $15 \mathrm{AU}$ per year. 


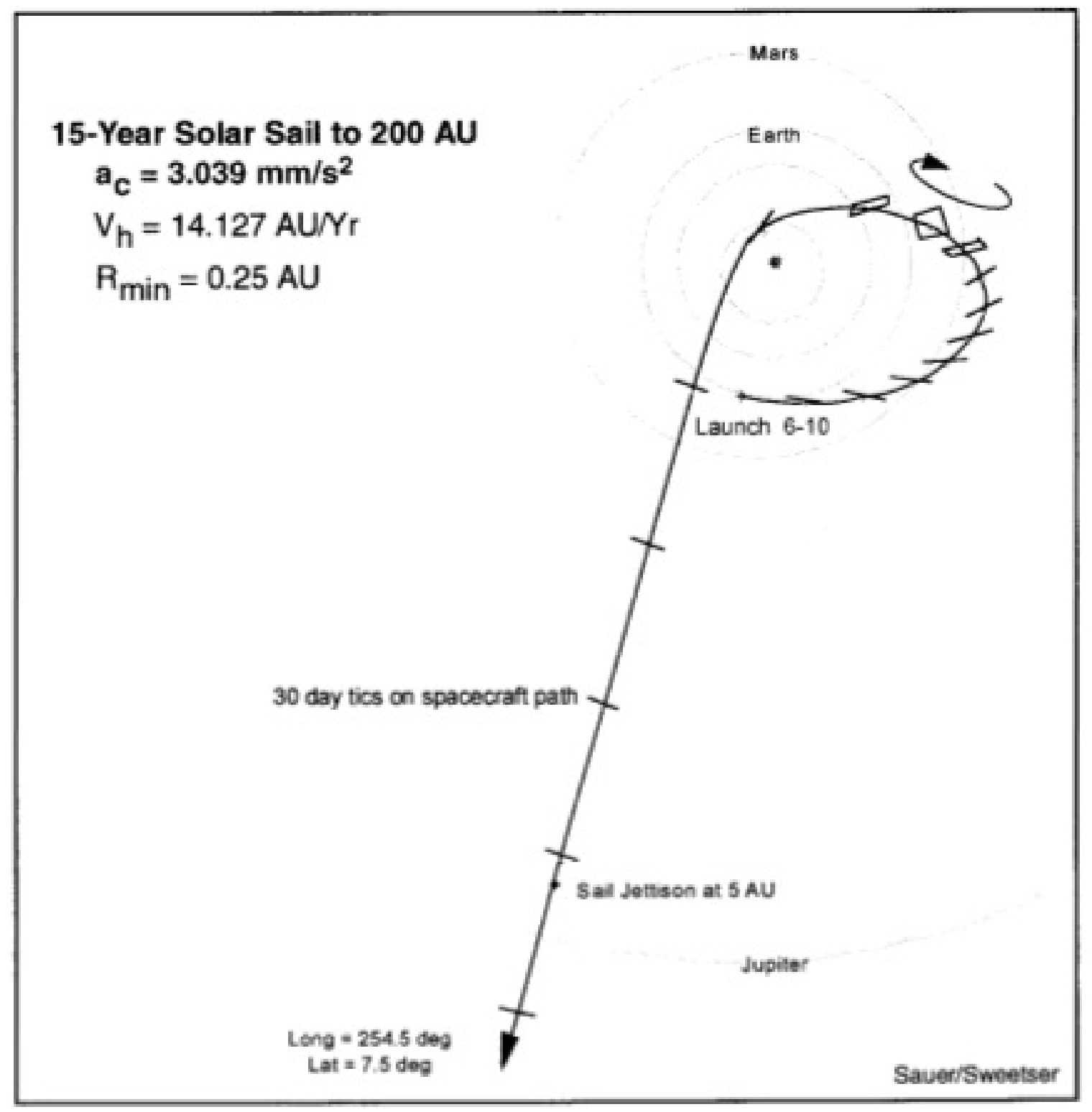

Figure 2.2: 1999 NASA Interstellar Probe Trajectory and Solar Sail Maneuvers[6]

\subsubsection{Keck Institute for Space Studies (KISS) Interstellar Probe Study}

The Keck Institute for Space Studies began a workshop in 2014 to study the necessary steps and possible spacecraft designs to reach the interstellar medium (ISM) over 200 AU from the sun[21]. The workshop presented the team with a Design Reference Mission (DRM 1.0). This mission would launch on the Space Launch System (SLS) 
in the 2020s, perform a Jupiter gravity assist and subsequent perihelion propulsive maneuver at 3-4 solar radii, achieve a solar system escape velocity of over $13 \mathrm{AU} / \mathrm{yr}$, and reach the local interstellar medium (LISM) within 10 years and the "pristine interstellar medium (ISM)" within 20-30 years.

The workshop set several ground rules and assumptions for the spacecraft, including total spacecraft mass of $725 \mathrm{~kg}$ and $400 \mathrm{~W}$ power supply. The results traded solar sail, electric sail, and Hall thruster propulsion systems for their designs. The team also traded trajectories, shown in Figure 2.3, examining simple Earth to Jupiter gravity assist using a low thrust propulsion system along the way, as well as examining a low perihelion propulsive maneuver with an optional Saturn flyby. This spacecraft would employ an Enhanced Multi-Mission Radioisotope Thermoelectric Generator (eMMRTG) to generate roughly $450 \mathrm{~W}$ of power, an estimate the team believed would be attainable within the project timeline. The spacecraft assumes and recommends technological developments in certain areas to make the designs feasible. For example, the solar sail design examines two cases, $3 \mathrm{~g} / \mathrm{m}^{2}$ and $10 \mathrm{~g} / \mathrm{m}^{2}$. The study states that although current solar sail technology (2014) produces sails with areal densities around $25 \mathrm{~g} / \mathrm{m}^{2}$, the authors assume an advancement in this area of technology. 


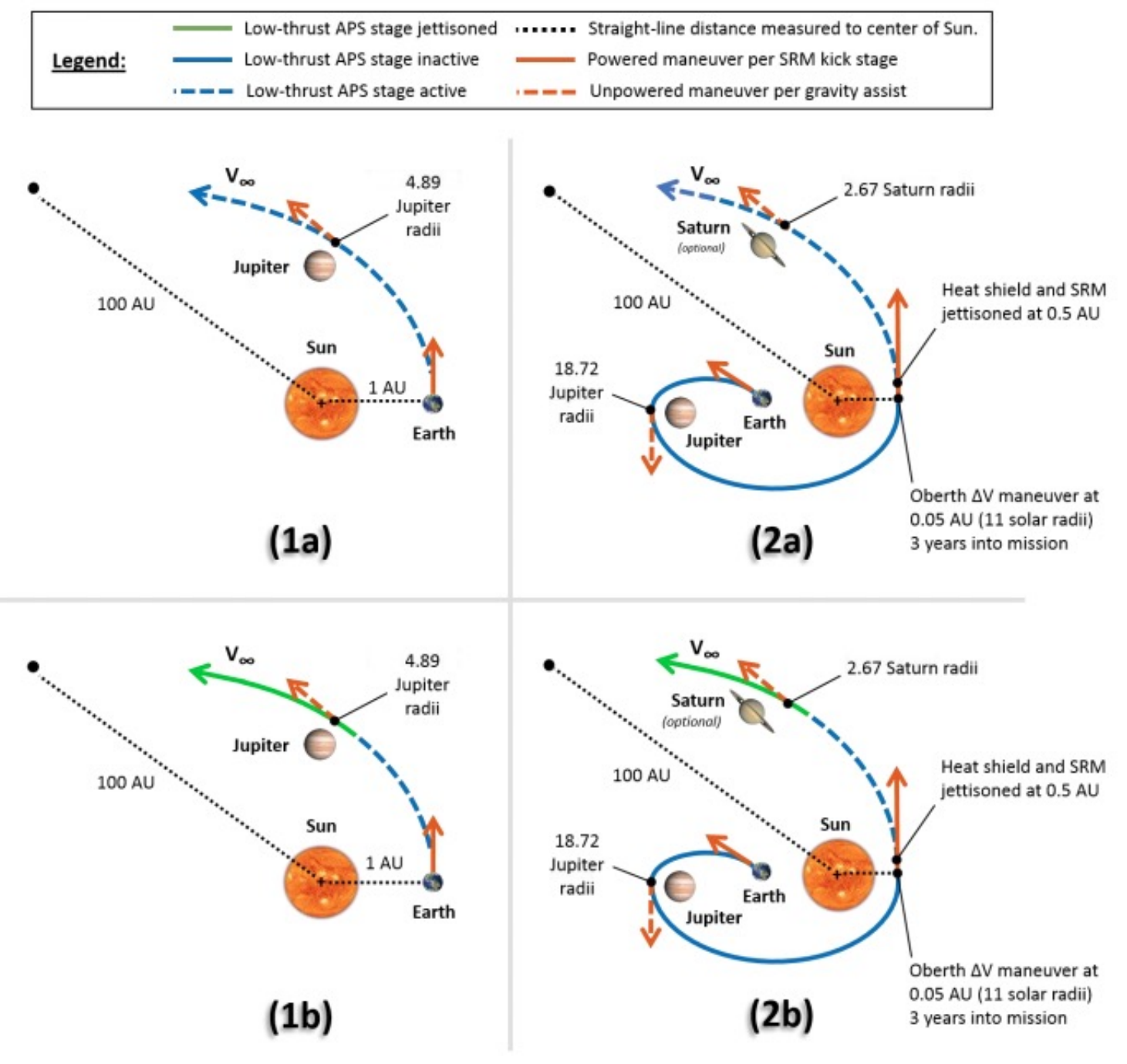

Figure 2.3: KISS Interstellar Probe Trajectory Solutions; a) Solutions
using Hall Thruster, b) Solutions using E-sails [7]

The KISS workshop is one of the very few studies to have mentioned the possibility of a small spacecraft using a parabolic solar concentrator to generate electrical power. In the study report titled Science and Enabling Technologies for the Exploration of the Interstellar Medium, the authors probe the idea of a $50 \mathrm{~m}$ x $50 \mathrm{~m}$ parabolic concentrator dish at $200 \mathrm{AU}$ to generate power[21]. Assuming a $70 \%$ concentrator efficiency and $25 \%$ solar cell efficiency, they estimate power generation to be around $15 \mathrm{~W}$. It is mentioned that the sail may be used as a communications antenna. 


\subsection{Enabling Technologies}

There is a convergence of several technologies that can make the solar powered deep space probe a reality. Payload requirements for deep space missions require little power and can be operated infrequently. In addition, new advanced nano-satellite electronics are constantly lowering the power requirements while maintaining performance. The combination of low payload and bus power consumption results in a low power spacecraft that can run on only a couple Watts at distances of 100 AU. Furthermore, these advanced nano-satellite electronics are beginning to see interplanetary travel with many nano-satellite missions planned to travel farther than Low Earth Orbit (LEO). The emergence of interplanetary CubeSats will soon demonstrate the capability of low cost systems to satisfy deep space mission requirements. Another key technological development that contributes to the feasibility of this concept is the recent improvements in small satellite deployable systems. Solar sails are increasing in size while simultaneously packing into smaller volumes, effectively reducing the ballistic coefficient and improving solar sail performance. In addition to solar sails, deployable reflector antennas are seeing improvements, providing small and low-power satellites with large aperture solutions to address communications issues from large distances. Improvements in these key areas can help realize the solar-powered deep space probe concept.

\subsubsection{Interplanetary CubeSats}

While CubeSats are well-known for cheap access to LEO, their capabilities are evolving and many interplanetary CubeSat missions are currently being developed. The first interplanetary CubeSats will be a pair of spacecraft accompanying NASA JPL's Insight missions to Mars. MarCO-A and B will fly by Mars to monitor the Insight spacecraft during its landing on the Martian surface, demonstrating the performance 
of nano-satellites in deep space[1]. While the two small spacecraft are considered CubeSats, it should be noted that these spacecraft are roughly $6 \mathrm{U}$, in comparison to traditional LEO CubeSats that can be as small as $1 \mathrm{U}$ in size. In addition to MarCO, many more CubeSats planned to travel past LEO are in development such as INSPIRE[22], Lunar Flashlight[23], NEAScout[24], LunarIceCube[25], and LunaHMap[26]. While these spacecraft have ambitious goals of operating in deep space, they plan to achieve these goals using readily available COTS components, resulting in much lower cost interplanetary space systems. In addition, these small spacecraft can employ rideshare architectures with other interplanetary spacecraft, further reducing costs. Although CubeSats have yet to reach OSS-like distances from Earth, technological demonstrations of their interplanetary capabilities are on the way and improvements are expected in the future.

\subsubsection{Low Power NanoSatellite Electronics}

The combination of recent technological developments, as well as the relatively low payload requirements for performing useful measurements on a dedicated OSS mission shows promise in the use of solar-powered small spacecraft for these missions. Payload power and operations frequency requirements for taking useful measurements of this OSS environment are, in fact, quite low. The instruments required to measure these changes in OSS environment can be boiled down to simply a plasma spectrometer and a magnetometer. Because the objective of the mission is to measure over large distances, the instruments may be operated infrequently, allowing the bus to remain in standby mode for a majority of the mission, thus drawing minimal average power. Other instruments such as a charged particle detector or a dust counter in the form of a camera or a microphone could be useful in characterizing this environment, although it would increase power requirements. These instruments have also decreased in size and power consumption. The INSPIRE CubeSat will include a $500 \mathrm{~cm}^{2}(0.5 \mathrm{U})$ 
magnetometer that is sensitive to less than $10 \mathrm{pT}[22]$. These advanced instruments will allow for a smaller and less powerful spacecraft bus to support these components.

New developments in low power nano-satellite electronics have also played a crucial role in enabling small satellite deep space missions. With the ongoing development of CubeSat electronics, bus and payload components now come in smaller sizes and operate on much lower power requirements. Low standby power modes for certain components allow for small spacecraft to run on low power consumptions, reducing the need for large solar panels, large batteries, or even nuclear power sources. Table 2.2 shows standby powers for some current commercial-off-the-shelf (COTS) bus components required to operate a spacecraft.

\section{Table 2.2: Bus Component Standby Power Consumption}

\begin{tabular}{cc}
\hline Component & Standby Power Consumption (W) \\
\hline Cube Computer (CubeSpace) & $0.1300[27]$ \\
NFSS-411 Sun Sensor (NewSpace Systems) & $0.0375[28]$ \\
UHF/VHF Transceiver (ISIS) & $0.2000[29]$ \\
\hline Total Standby Power: & 0.3675 \\
\hline
\end{tabular}

With standby values for bus components under $400 \mathrm{~mW}$, power requirements for small spacecraft are significantly more feasible to design to. Interstellar missions have similar power requirements in that the spacecraft must have a low power consumption due to limited availability of power at Voyager-like distances from the Sun, even with the use of RTGs. Due to the length of time it takes to reach distances over 100 AU, RTG power generation decreases significantly from its original power generation values. 


\subsubsection{Small Sat Deployable Technologies}

Deployable space technologies are attractive options for a number of reasons. Due to fairing limitations, some structures are simply too large to launch in the nominal configuration. Folding these structures and decreasing the maximum dimension allows for the structures to retain the desired size and fit within a typical launch vehicle fairing. For example, the James Webb Space Telescope (JWST) is a space telescope consisting of a 6.5 meter diameter primary mirror and an even larger $22 \mathrm{~m}$ $\mathrm{x} 12 \mathrm{~m}$ sunshield to keep the optics cold. This spacecraft will launch on an Ariane 5 launch vehicle with a maximum fairing width of around $5 \mathrm{~m}$. This clearly requires the JWST to fold its primary mirror and package the large sunshield into a compact compartment[30].

Deployable systems are of particular interest to small spacecraft, such as CubeSats, as they deploy from small compartments called PPODs. Small spacecraft also lack large bus dimensions for body-mounted components such as solar panels or antennas. Deployable systems for these types spacecraft make efficient use of their small volumes and can greatly improve subsystem performance in areas of communications, power generation, and even propulsion. As small spacecraft graduate into the interplanetary regime, solutions must be developed to communicate over larger distance and generate power with lower solar intensity.

Fortunately, deployable technologies for small spacecraft are improving, and many companies and organizations are researching, developing, and selling different deployable technologies from deployable solar panels to deployable reflector antennas. Unfortunately, deployable solar panels are not effective in deep space, so this paper will not be exploring deployable solar panel techonologies for small spacecraft. Instead, deployable sail and antenna technologies will be examined to determine how these systems can apply to an interstellar probe. Due to the large distances from the Sun that 
these spacecraft experience, large communications dishes will be necessary to communicate with Earth and an unconventional method of generating solar power will be required to satisfy power requirements. Power generation can be accomplished with a large parabolic dish that concentrates the low intensity sunlight onto solar panels, which can conveniently be used as a communications dish as well. These operations can be completed with a large deployable parabolic dish system.

Several deployable systems exist today and many more are in development. Drag sails, solar sails, and deployable antennas are of particular interest to this thesis, as increasing packing efficiencies in these areas provide promise that large deployable parabolic systems can be available for this type of mission in the near future. Practical solar sails have flown since 2010, beginning with the IKAROS mission, a 300kg spacecraft with a $14 \mathrm{~m} \times 14 \mathrm{~m}$ solar sail that flew to Venus within 7 months[31]. Solar sails have since been flown on increasingly smaller spacecraft to maximize the ballistic coefficient and maximize the effect of the solar sail. The Planetary Society has flown a $3 \mathrm{U}$ CubeSat with a $32 \mathrm{~m}^{2}$ solar sail in 2015, successfully deploying the solar sail and declaring the test flight a success[32]. Drag sail development is also increasing as the concern for space debris grows. These sails increase the drag force imparted on Low Earth Orbit (LEO) spacecraft and decrease the time it takes to return to Earth. Drag sails, much like solar sails, must be large in order to maximize the effect on the spacecraft, resulting in an effort to maximize the size to weight ratio of the deployable system.

On the other hand, deployable communication systems present efficient solutions to employ large communications apertures from small volumes and increase the performance of the system. Deployable communications systems come in various forms such as phased array antennas and parabolic dishes. As opposed to solar sail and drag sail systems, deployable antenna systems often have lower packing densities due to the required shape of these systems. Reflector antennas require that the deploy- 
able system or the feed be offset from the main body of the spacecraft rather than deployed as a planar surface. Nonetheless, this paper will explore solutions involving both types of deployable technologies. 


\section{Chapter 3}

\section{LIGHT ANALYSIS AT 100 AU}

In order to determine the subsystems a solar-powered small satellite will need in deep space, simple analysis of operating at $100 \mathrm{AU}$ can shed light on the main requirements and possible solutions. $100 \mathrm{AU}$ is chosen as the upper limit of the operating distance for a number of reasons. This is an ambitious distance for a non-nuclear powered spacecraft and a great step for proof-of-concept missions. JUNO, a spacecraft currently orbiting Jupiter at roughly $5 \mathrm{AU}$, is the furthest spacecraft from the sun that has operated without RTGs[33]. $100 \mathrm{AU}$ is also a scientifically interesting distance from the sun. The termination shock region stretches from about 80 - $100 \mathrm{AU}[19]$, so this target distance should allow this type of spacecraft to measure the transition into the heliosheath and provide useful data. The next interstellar region is edge of the heliopause at 120 - $150 \mathrm{AU}$, a significant increase in distance. Designing a spacecraft

to the edge of the heliopause will require much larger systems that likely will not be available within the near future (10 - 20 years). Therefore, designing a solar-powered probe to reach $100 \mathrm{AU}$ is an ambitious but reasonable stepping stone to prove this technology.

\subsection{Power Generation}

Power sources for deep space missions are almost exclusively dominated by the radioisotope thermoelectric generator (RTG) due to the impracticality of solar cells at large distances from the sun. This nuclear power source provides power at any distance from the sun, decreasing in power generation over time as a function of the half-life of the radioactive fuel. While nuclear power sources are ideal for deep space 
missions, they carry high costs and safety risks. If the RTG container leaks, it risks contamination of the spacecraft. In addition, launch failures from launch vehicles carrying RTGs risk contaminating Earth. RTGs also require immense amounts of paperwork as a result of regulations related to these safety concerns. Small spacecraft, especially CubeSats, are also known for their low-costs, often employing commercialoff-the-shelf (COTS) parts. Utilizing a nuclear power source would negate these low costs. Furthermore, if the spacecraft employed a rideshare architecture, the nuclear power source would present a risk of compromising the primary spacecraft and mission. Determining a non-nuclear power source solution for these small deep space probes would reduce cost and risk for the spacecraft and mission.

The alternative to nuclear power is the use of solar power. At distances of 100 $\mathrm{AU}$, the solar intensity is only $0.1367 \mathrm{~W} / \mathrm{m}^{2}$, requiring infeasibly large solar panels to generate enough solar power, even with state-of-the-art solar cell technology. Instead, small spacecraft can employ a solar concentrator, using similar technologies as the deployable solar sails and antenna systems. If deployed in a parabolic shape, the low intensity sunlight can be concentrated onto solar panels mounted on the spacecraft. This requires smaller solar panel areas and still enables the spacecraft to capture large amounts of this dim sunlight. Light analysis of this method of power generation shows that it is feasible given the appropriate deployable systems. Figure 3.1 shows the power received at different stages of the power generation process given certain inefficiencies. The blue curve represents the sunlight that hits the concentrator dish. This should simply be cross-sectional area facing the sun. The solar concentrator dish is assumed to have aluminized Mylar as its reflective surface, and although its reflectivity can be over 95\%[34], the concentrator efficiency is assumed to be $80 \%$. The $80 \%$ of the sunlight that reflects off the concentrator is then assumed to hit the solar panel. Here, several inefficiencies are taken into account. The solar panel is assumed to have a $92.5 \%$ packing factor efficiency, $92.5 \%$ average incidence angle efficiency, 
and solar cell efficiency of $30 \%$. The average incidence angle efficiency equates to about 22.3 degrees of incidence angle.

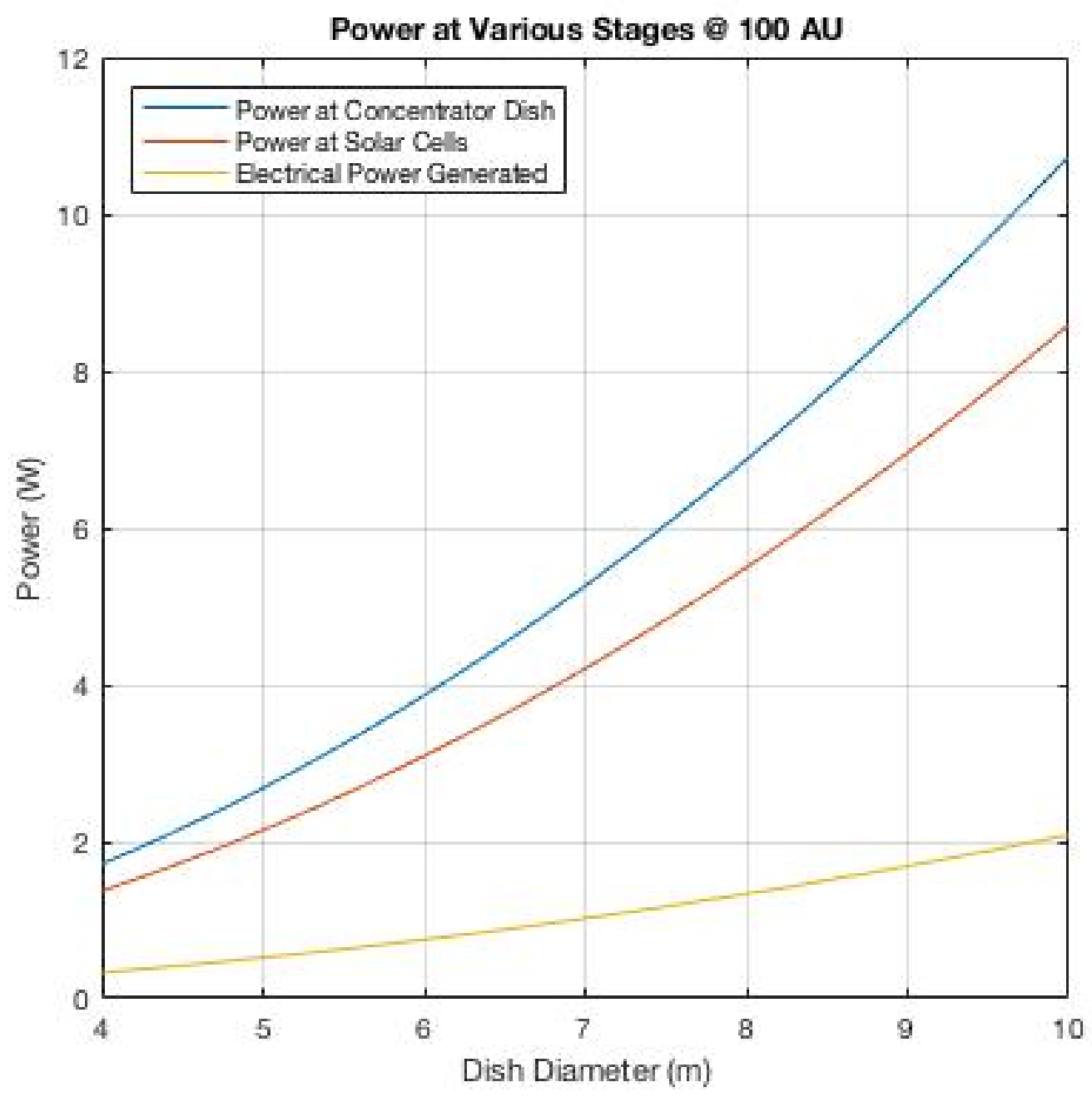

Figure 3.1: Power Received at Various Stages During Power Generation Processes

This graph shows that even with a relatively large dish, little electrical power can be generated. For example, with a 7 meter dish diameter, this process can only generate 1 Watt of electrical power. However, if bus and payload power requirements are low enough, this method can generate a sufficient amount of power. Fortunately, OSS missions do not require high power and high frequency payload operations. Coupling this with new advanced low power nano-satellite electronics, standby power 
modes can reach low power consumption levels of less than $400 \mathrm{~mW}$ as detailed in Table 2.2. If the spacecraft were to remain in standby mode for most of its mission, the spacecraft could simply charge batteries and store energy for its high-power processes. With sufficient deployable systems, this power generation method could be feasible for these types of spacecraft and missions.

\subsection{Radio Frequency Communication}

If we assume the power generation scheme mentioned above, we conveniently have a large dish that we can also use for communications back to Earth. As previously mentioned, the study of the OSS environment does not require frequent measurements, meaning data volumes that must be transmitted back to Earth are small. Therefore, transmissions can be once a day or even once a week. However, even with a large dish, communicating over $100 \mathrm{AU}$ requires a significant amount of both electrical and radio frequency $(\mathrm{RF})$ power. Reducing the bit-rate can help close the link with less power. Quick analysis of communications requirements shows that it is possible to close the link, albeit with a relatively high RF power. Table 3.1 shows the various parameters and efficiencies assumed for a general analysis of a traditional deep space communications link. These numbers are consistent with traditional parameters and efficiencies for this type of link. There are several options to communicate with Earth from deep space. The Deep Space Network (DSN) operates on S, X, and Ka-band frequencies[35], supporting the frequencies with several different size dishes. There is also a fourth option of using Ultra High Frequency (UHF) with Stanford SRI's $45.7 \mathrm{~m}$ dishes[36]. Table 3.2 details the available ground station solutions with the accompanied frequency and receiving dish sizes.

Using these parameters, calculating the required RF power as a function of the transmit dish size with traditional link equations provides useful trends to determine 


\section{Table 3.1: Communications Parameters for Deep Space Link}

\begin{tabular}{cc} 
Parameter & Value \\
\hline Signal-To-Noise Ratio & $10 \mathrm{~dB}$ \\
Signal Link Margin & $3 \mathrm{~dB}$ \\
Data Rate & $10 \mathrm{bps}$ \\
Transmitter Efficiency & 0.35 \\
Receiver Efficiency & 0.7 \\
System Noise Temperature & $75 \mathrm{~K}$ \\
Transmit \& Receive Antenna Pointing Loss (each) & $-3 \mathrm{~dB}$ \\
Polarization Loss & $-2 \mathrm{~dB}$ \\
Line Loss & $-2 \mathrm{~dB}$ \\
Implementation Loss & $-3 \mathrm{~dB}$ \\
Atmospheric Loss & $-0.3 \mathrm{~dB}$ \\
\hline Total Losses before Space/Noise Losses & $-10.3 \mathrm{~dB}$ \\
\hline
\end{tabular}

Table 3.2: Deep Space Ground Station Options

\begin{tabular}{c|cccc}
\hline & DSN & DSN & DSN & Stanford SRI \\
\hline Frequency & $2.2 \mathrm{GHz}$ & $8.4 \mathrm{GHz}$ & $32 \mathrm{GHz}$ & $400 \mathrm{MHz}$ \\
Dish Size & $70 \mathrm{~m}$ & $70 \mathrm{~m}$ & $34 \mathrm{~m}$ & $45.7 \mathrm{~m}$ \\
\hline
\end{tabular}

the ideal dish size for a feasible transmission power. Figure 3.2 shows the required RF power for various transmit dish sizes for the four ground station options. 


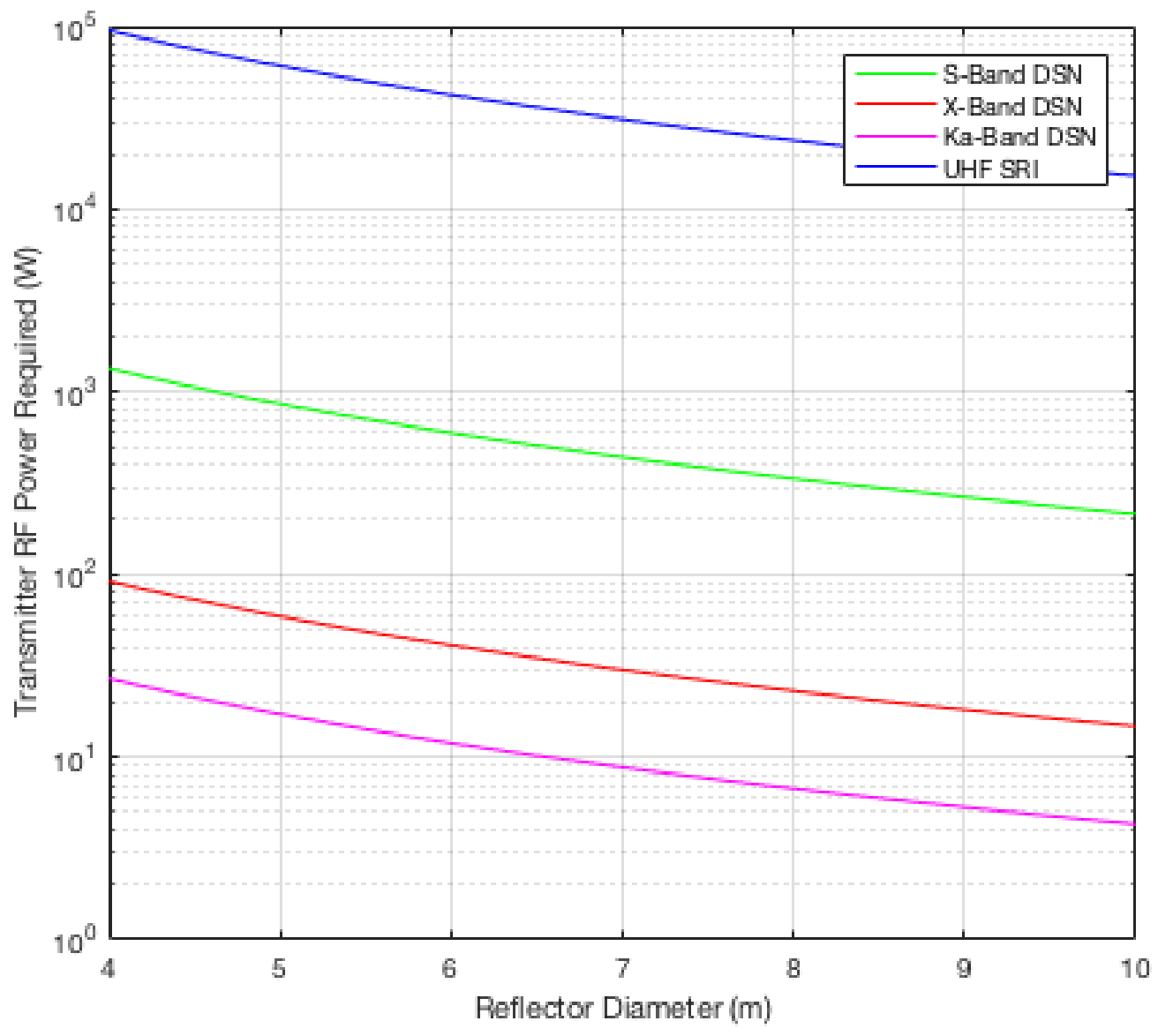

Figure 3.2: RF Power Required vs. Reflector Dish Size at 100 AU

The trend lines show that X-Band and Ka-Band are the more attractive options for communicating from 100 AU distances. The UHF option with the Stanford SRI dishes is simply to low in frequency to transmit enough data with reasonable transmitter power. S-Band also requires too much power, even with large reflector dishes. The lower frequency options may be useful at closer distances, however, they require too much RF power at $100 \mathrm{AU}$, on the order of hundreds or even thousands of Watts of $\mathrm{RF}$ power. 


\section{Chapter 4}

\section{SYSTEM REQUIREMENTS AND OVERVIEW}

Several requirements must be established to understand what the spacecraft requires to operate along its mission. These requirements will also help convey why this type of spacecraft may be useful to the scientific community and spacecraft and mission design engineers. Determining a useful scientific mission and what payloads and operations will be required to support this scientific yield will help determine bus requirements.

The spacecraft as a whole has several requirements that must be met in order to maintain its low-cost and small size quality. These are outlined in Table 4.1. This spacecraft is largely meant to be an auxiliary to a primary mission or as a collection of a large quantity of these spacecraft. This idea is to be able to visit multiple places in the OSS with only one mission. The small mass and packaged dimension requirements

allow this spacecraft to ride along a primary mission as a secondary payload or allow many of these spacecraft to pack into one host for a swarm architecture mission. The mass and packaged dimension requirements act more as a goal rather than a hard requirement, with the aim of generally lowering spacecraft cost and optimizing the spacecraft for rideshare possibilities. Limiting the power source to a non-nuclear option will reduce overall spacecraft cost and also reduce risk of the secondary payload affecting the primary spacecraft, if a rideshare system is used.

To determine the right payloads, we must understand what data must be taken in these deep space environments. Primarily, scientists are interested in the interaction between the interstellar medium and our solar system. This manifests into changes in magnetic field direction and differences in plasma environment. To take these measurements, a magnetometer and plasma spectrometer are required. Both of these 
Table 4.1: Overall Spacecraft Requirements

\begin{tabular}{|c|c|}
\hline Maximum Mass & $30 \mathrm{~kg}$ \\
\hline Maximum Packaged Dimension & $50 \mathrm{~cm}$ \\
\hline Power Source & Non-Nuclear \\
\hline Max. Nominal Operation Distance & $100 \mathrm{AU}$ \\
\hline
\end{tabular}

instruments have been miniaturized and engineered into the CubeSat form, reducing mass, volume, and power requirements[22]. Basic data volume requirements have been set to characterize the scientific mission for the spacecraft and are detailed in Table 4.2. These requirements are not optimized, however, they will provide a good sense of what the resulting spacecraft design will look like for a reasonable scientific data requirement.

Table 4.2: Science Payload and Data Requirements

\begin{tabular}{|c|c|}
\hline Scientific Payload (minimum) & Magnetometer \& Plasma Spectrometer \\
\hline Payload Operation & 4 mins/hour (paired operation) \\
\hline Data Volume per Transmission & $1 \mathrm{~KB}$ \\
\hline Transmission Frequency & 1 Day -1 Week \\
\hline
\end{tabular}

The magnetometer and plasma spectrometer represent the minimum instruments required to return useful data from the OSS environment. Other instruments may be added to the spacecraft, although, it will increase the average power and affect spacecraft configuration, among other considerations. The instruments will need to operate at the same time, since pairing the two measurements allows for better characterization of the changes in environment between the interstellar regions. Of the currently operating interstellar probes, Voyager 2 is the only spacecraft with both instruments operating properly. The plasma spectrometer on Voyager 1 was turned off before reaching the termination shock region due to degradation of performance. New 
Horizons is lacking a magnetometer due to budget and time constraints and, therefore, will need to infer this data from other instruments. These two instruments cover a major portion of what is necessary to take useful measurements and characterize these interstellar regions.

\subsection{System Overview}

The overall design of the spacecraft was the result of many iterations, and there are many points to begin explaining the nature of the engineering problem that this spacecraft resulted from. In order to give a clear idea of the relevant requirements analyses and decisions made, the overall spacecraft will be displayed first. The analysis and decisions made to reach this design will follow.

The spacecraft components and configuration are detailed in this section. Many components are based off COTS products currently available for nano-satellites. Several components may need to be custom-made specifically for this spacecraft, especially the deployable system. A block diagram of data and electrical power paths can be seen in Figure 4.1. The block diagram details all of the basic components and subsystems present in the spacecraft as well as the interactions between these subsystems. 


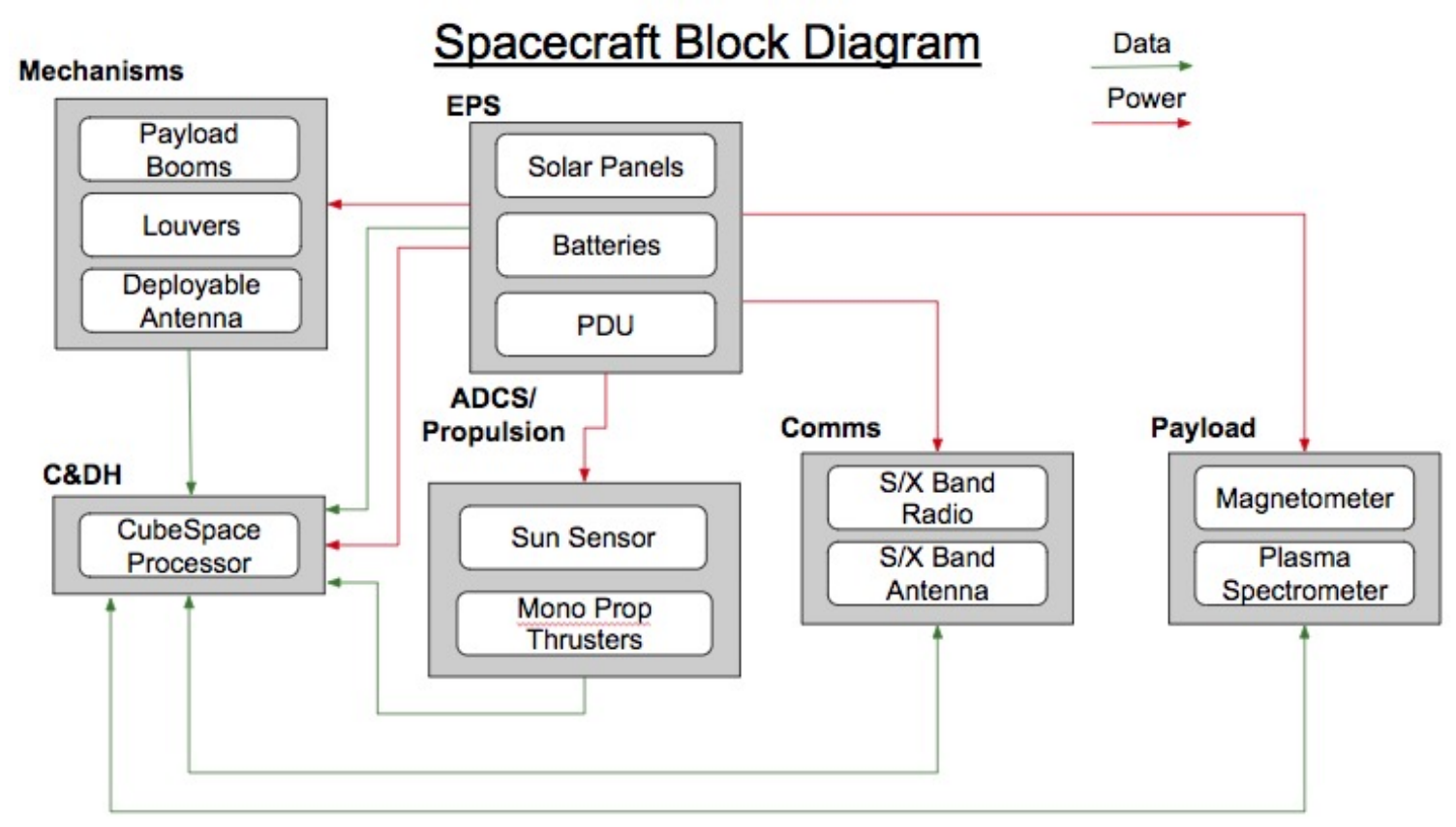

Figure 4.1: Overall Block Diagram of Spacecraft Subsystems

\subsubsection{Concept of Operations}

It is important to understand the concept of operations for this unconventional spacecraft. As mentioned before, the spacecraft relies on low standby power and infrequent measurements and transmissions to operate using solar power in deep space, keeping average power consumption low. However, there are several events that occur before nominal operations and after deployment from the host spacecraft.

Shortly after deployment from the host spacecraft, the small OSS probe will begin spin up to initiate spin stabilization in order to stay inertially pointing and minimize the need for active attitude control. After spin up, the small pre-flyby burn will be executed, which will consist of a total burn time of 14 minutes to perform a $10 \mathrm{~m} / \mathrm{s}$ delta-V maneuver on the $30 \mathrm{~kg}$ spacecraft. This burn will be using two of the $100 \mathrm{mN}$ Busek BGT-X1 monopropellant thrusters. This assumes that each thruster will be operating at the maximum throttleable thrust range of $180 \mathrm{mN}[37]$. This burn can oc- 
cur in one 14 minute burn, or it can be divided into several burns to avoid overheating from the hot catalyst bed. After the impulsive maneuver, the spacecraft will deploy its inflatable antenna and the payload booms. Once all deployments are complete, the spacecraft will perform final spin up maneuvers and then re-orient its spin axis towards the Earth to begin nominal operations. While payload measurements close to the Sun may be useful to calibrate the instruments, these measurements are not useful in characterizing the OSS environment. Therefore, payload deployment and operations can also occur later in the mission.

Nominal operations for the spacecraft consists of four power modes that are detailed in Table 4.3. Duty cycles are representative of a 7 day operations cycle. This means that transmissions to Earth occur once every week. As is evident by the duty cycles, the spacecraft spends most of its time charging its batteries in standby mode at a power consumption of roughly $0.3825 \mathrm{~W}$. For 4 minutes every hour, it will run the magnetometer and plasma spectrometer to collect measurements. After collecting data for a week, the spacecraft will undergo its highest power consumption during transmission, when the spacecraft must transmit a kilobyte of data back to Earth. Using the Deep Space Network's lowest downlink rate of 10 bps[35], this means the spacecraft will transmit for 800 seconds every week, or about 13 to 14 minutes. The final power mode during nominal operations will be during re-orientation towards the Earth. The frequency of the maneuvers can drop to a rate of one maneuver every 100 days. The operations cycle of one week is based off of the amount of electrical power generated at a distance of $100 \mathrm{AU}$. At closer distances, the spacecraft can operate its payload and transmit more frequently due to the higher availability of sunlight. Conversely, at farther distances, the spacecraft will need to operate payloads and transmit less frequently. The limit at which the spacecraft can operate will be determined by the usefulness of the data, which, if collected too infrequently, may be useless in characterizing the changes of the OSS environment. However, the physical 
limit of the spacecraft's operation will be when the available electrical power will drop below the power required operate in standby mode. The various power modes at 100 AU are detailed in Table 4.3.

Table 4.3: Power Breakdown for Spacecraft Power Modes at 100 AU

\begin{tabular}{|c|c|c|c|c|}
\hline & \multicolumn{4}{|c|}{ Power Modes } \\
\hline Component & Transmit (W) & Measurement $(\mathrm{W})$ & Standby $(\mathrm{W})$ & Burn $(\mathrm{W})$ \\
\hline \multicolumn{5}{|c|}{ Payload } \\
\hline Magnetometer & - & 2 & - & - \\
\hline Plasma Instrument & - & 2 & - & - \\
\hline \multicolumn{5}{|c|}{ ADCS } \\
\hline Sun Sensor & 0.13 & 0.13 & 0.0375 & 0.13 \\
\hline Thruster System & - & - & - & 4.5 \\
\hline \multicolumn{5}{|c|}{ Comms } \\
\hline Transmitter/Receiver & 76 & 0.2 & 0.2 & 0.2 \\
\hline \multicolumn{5}{|c|}{ C\&DH and EPS } \\
\hline Processor & 0.43 & 0.43 & 0.13 & 0.43 \\
\hline PDU & 0.015 & 0.015 & 0.015 & 0.015 \\
\hline Total Power & 76.775 & 4.775 & 0.3825 & 5.275 \\
\hline Duty Cycle (\%) & 0.13 & 6.77 & 93 & Infrequent \\
\hline
\end{tabular}

The power modes listed in Table 4.3 show the various power modes during nominal operations. The spacecraft spends most of its time in standby mode charging its batteries. Every hour for four minutes, the spacecraft turns on its payload of a magnetometer and plasma instrument to take measurements. Once per week, the spacecraft will transmit $1 \mathrm{~KB}$ of data back to Earth. At 100 AU, burns will occur roughly once every 100 days, therefore, the duty cycle is not displayed. It should be noted that the duty cycle for the burn mode increases and the power consumption of the radio decreases when closer to the sun, however, the power modes displayed 
detail the worst case power consumptions at a time when electrical power availability is at its lowest.

\subsubsection{Spacecraft Overview}

Now that the concept of operations for the spacecraft has been explained, we can now show the various configuration states of the spacecraft. The spacecraft has two main configuration states: undeployed and deployed, referring to the deployment of the inflatable antenna and the payload booms. These can be seen in Figure 4.2 and Figure 4.3. 


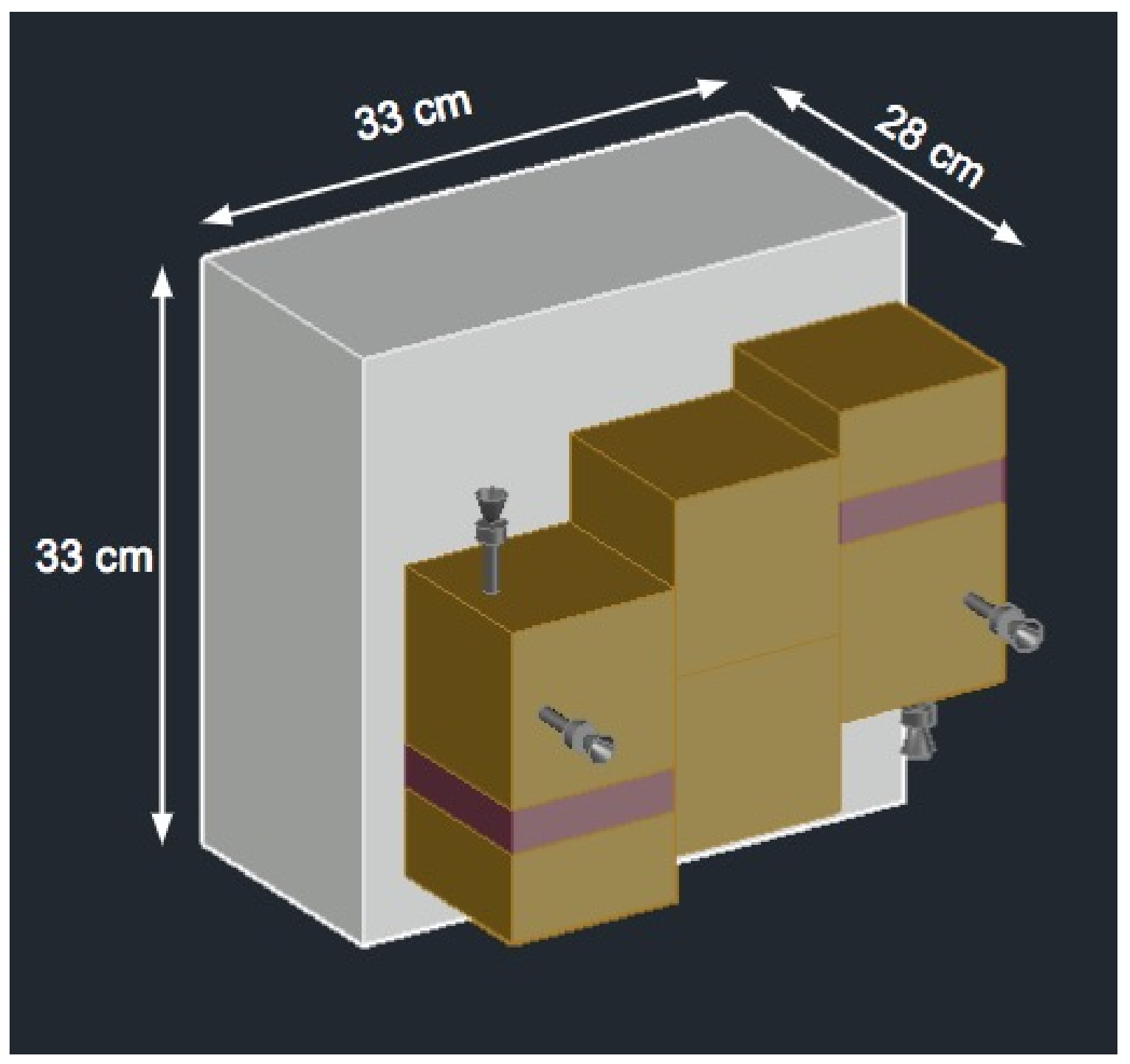

Figure 4.2: Spacecraft Without Deployed Mechanisms 


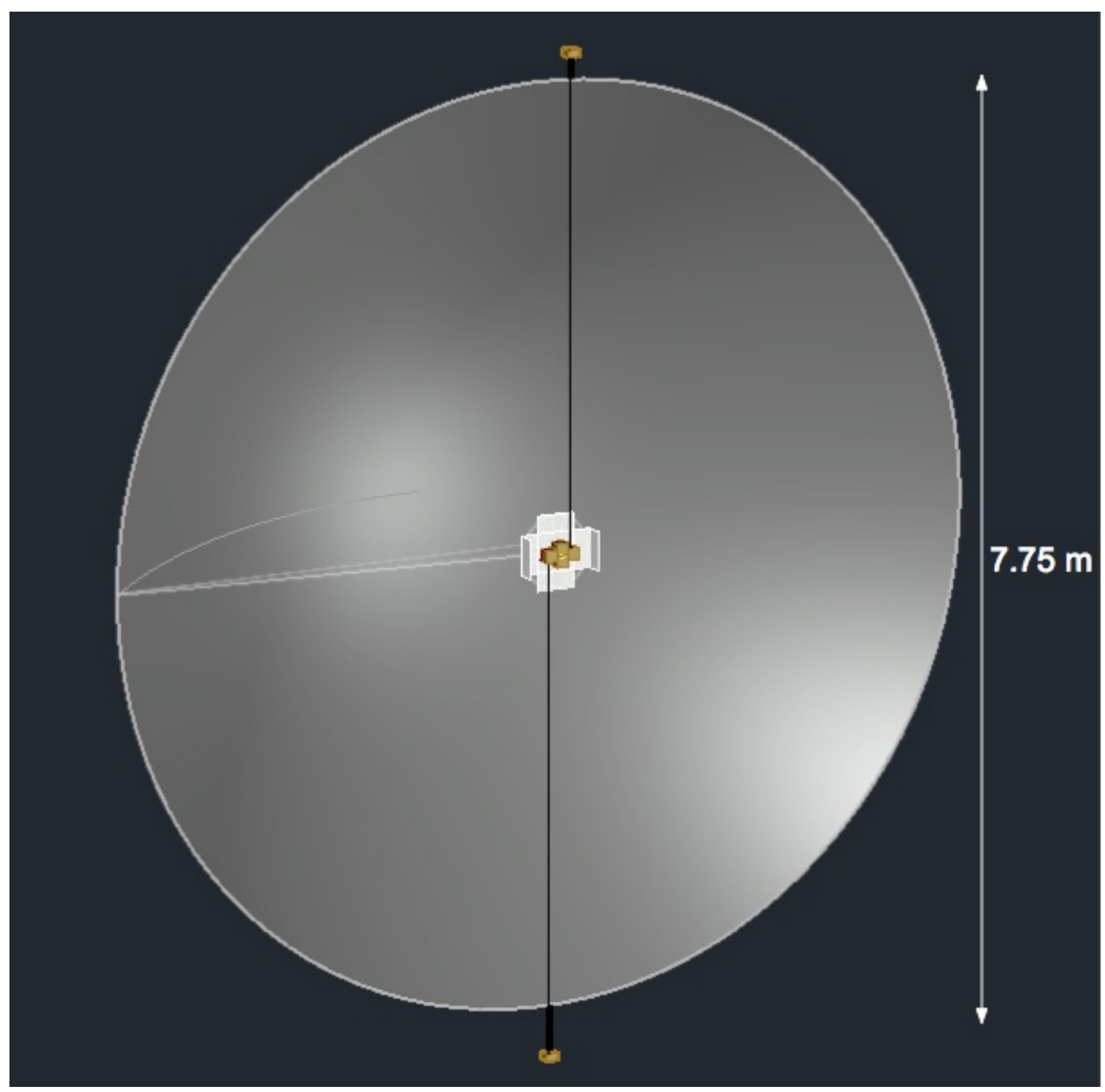

Figure 4.3: Spacecraft With Fully Deployed Inflatable Antenna, Radiator Panels, and Payload Booms

The undeployed state of the spacecraft shows a total of four $100 \mathrm{mN}$ mono propellant thrusters, two for the spin up maneuver and two for the $10 \mathrm{~m} / \mathrm{s}$ delta- $\mathrm{V}$ maneuver. Reasons for why these systems were chosen will be detailed in the ADCS and propulsion chapter, chapter 9 .

As seen in the deployed state of the spacecraft, the payload booms extend past the edge of the inflatable antenna. This is because the deployable dish would com- 
promise scientific measurements of the plasma instrument, as the dish would generate a "wake" as it travelled though interplanetary space. Because the magnetometer is similarly sized, both instruments are placed on booms to maintain symmetry for spin stabilization. Therefore, the instruments extend 0.25 meters beyond the edge of the dish. Whether the payload booms must extend the instruments even further must be investigated to ensure that the scientific data yield is accurate. However, with stateof-the-art lightweight booms that can weigh as low as 100 grams per meter[38], adding length to the booms would not add significant mass to the spacecraft. The spacecraft employs a $7.75 \mathrm{~m}$ diameter parabolic dish that serves to concentrate sunlight onto solar panels and act as a high gain antenna.

The inflatable antenna deploys in a unique fashion as the compartment that it is housed in serves two purposes. First, it acts as a simple compartment to house the inflatable dish. However, its second purpose is to act as deployable radiators to radiate extra heat from the solar panel and feed surface. Because the dish is sized for $100 \mathrm{AU}$ and must remain fully deployed for the duration of the mission, it will be severely oversized when closer to the Sun, concentrating excessive amounts of sunlight onto the spacecraft. The sequence of the deployment of the radiator panels can be seen in Figure 4.4. 


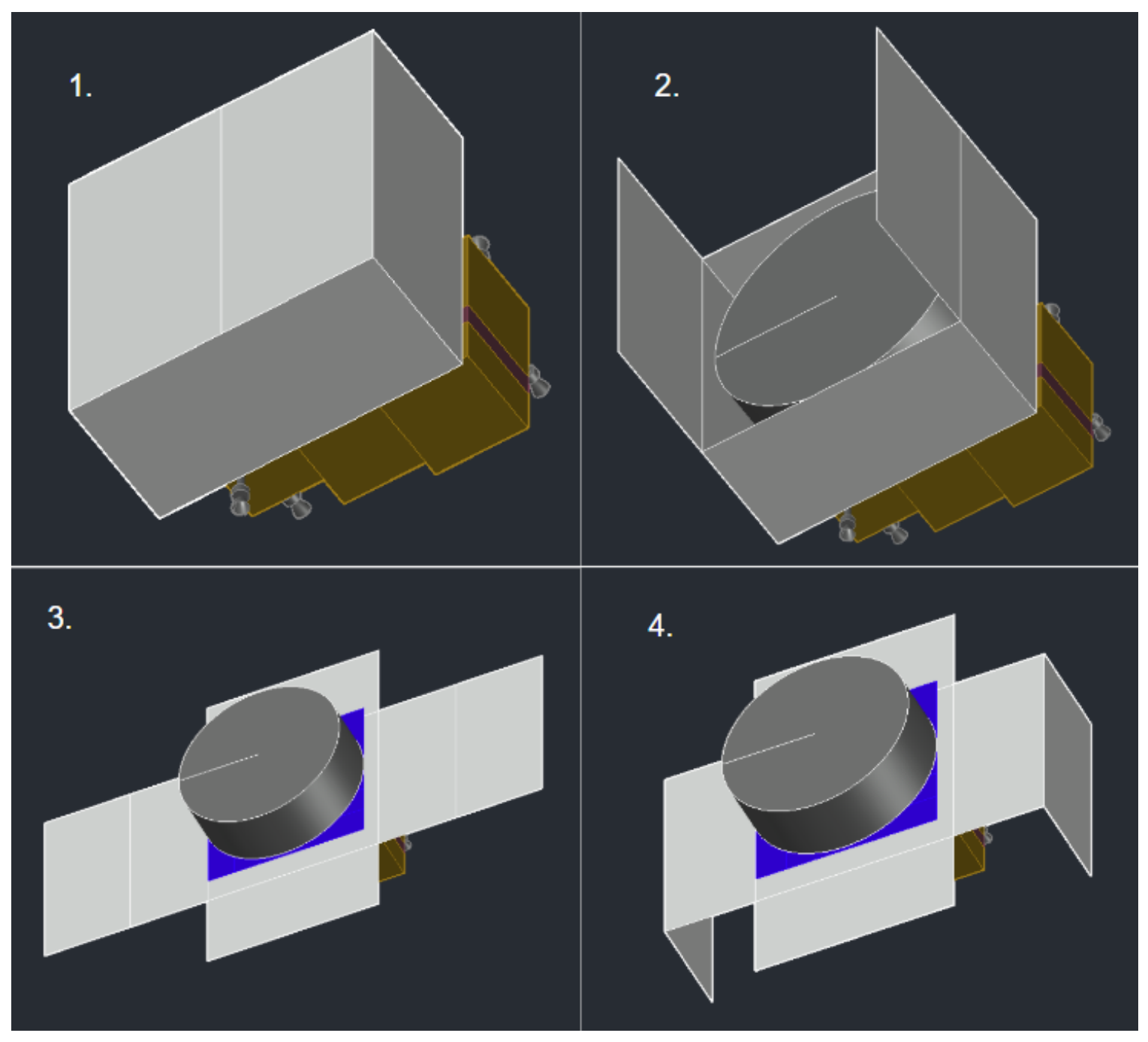

Figure 4.4: Deployable Radiator Deployment Sequence

Figure 4.4 shows how the housing of the inflatable antenna unfolds into extra radiator surface, revealing the inflatable antenna inside, represented as a cylinder. This will help keep the solar cells surface from overheating. The radiators are also overhanging in order to minimize the area blocking the RF waves reflected off the inflatable antenna.

The sequence of the deployment of the payload booms can be seen in Figure 4.5. 


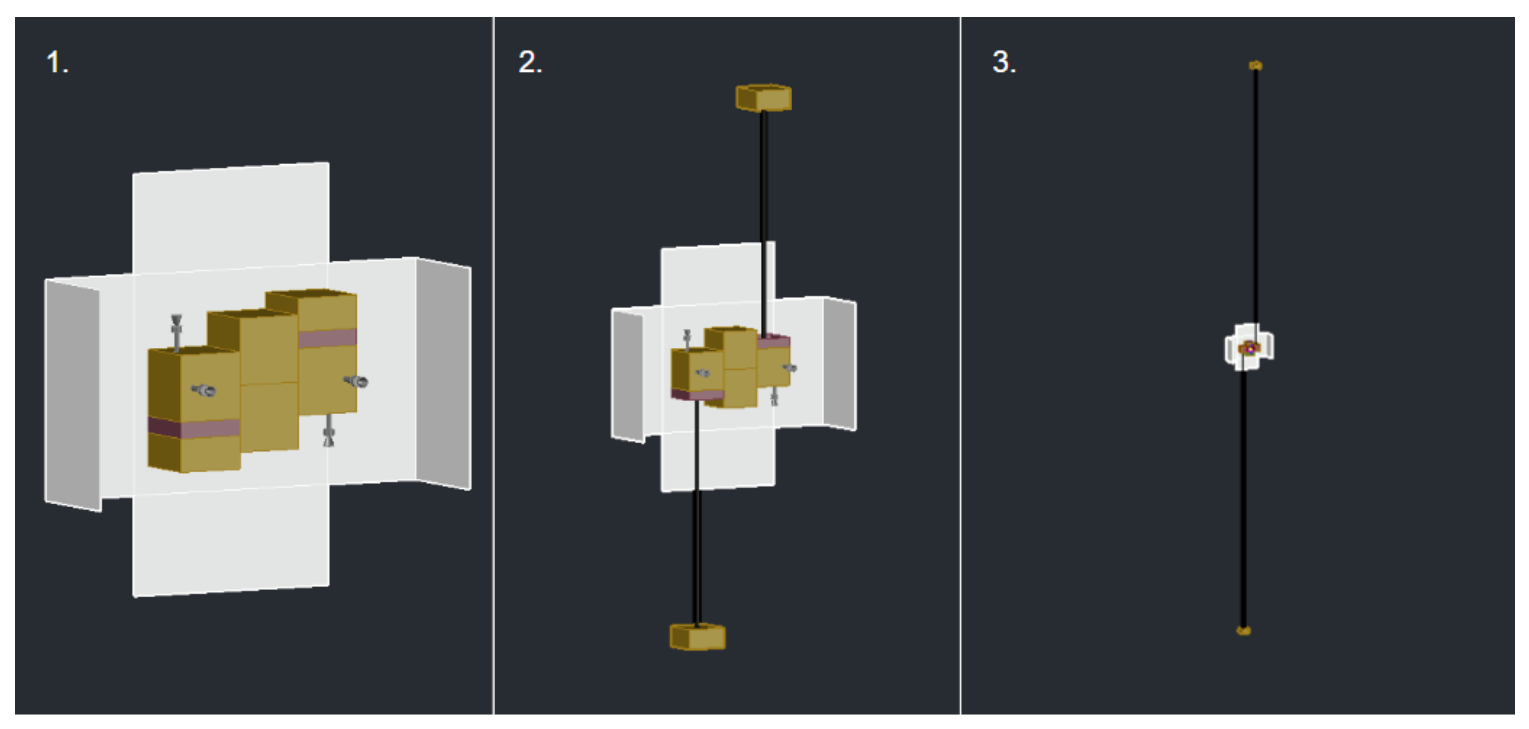

Figure 4.5: Payload Boom Deployment Sequence

Assuming each boom can be housed in a $0.25 \mathrm{U}$ compartment, consistent with the systems seen on Lightsail[38], the booms will deploy radially from the bus, with aid from the initial spin stabilization of the spacecraft.

The reasons for why these components and configurations were selected result from a number of key factors which will be explored in further detail in subsystem specific chapters. Many requirements also stem from the Voyager 1 reference mission trajectory. Reasons why this trajectory has been chosen will be explained in the orbital solutions chapter. However, this gives a brief overview of the conclusions that have been reached. 


\section{Chapter 5}

\section{ORBITAL TRAJECTORY OPTIONS}

The orbital trajectory of an OSS probe is crucial for this type of mission. The OSS probe design examined in this paper is largely intended to be an auxiliary spacecraft, as its low mass and volume makes it ideal as a secondary payload. For this reason, this chapter will examine possible orbital solutions for a secondary payload.

The spacecraft must end up on an orbital trajectory with enough energy to reach solar system escape velocities. These trajectories require powerful launch vehicles and often require a gravitational assist maneuver to increase the heliocentric velocity of the spacecraft. The Voyager and Pioneer probes both used multiple flyby maneuvers of the two largest planets in our solar system, Saturn and Jupiter, as a means to maximize their heliocentric velocity without the use propulsive delta- $\mathrm{V}$ maneuvers. Voyager 1 is the faster of the objects, travelling at $17 \mathrm{~km} / \mathrm{s}$ or $3.6 \mathrm{AU} /$ year. Even at these speeds, the probe reached 100 AU in over 30 years. New Horizons was the first object to be directly injected into a solar system escape trajectory, however, without the Jupiter flyby, it would have taken much longer to reach 100 AU. The Jupiter flyby in 2007 increased the probe's speed to just under $16 \mathrm{~km} / \mathrm{s}[16]$, comparable to the Voyager and Pioneer probes.

Throughout past interstellar probe studies, there has been an effort to minimize this travel time by employing new methods to reach significantly greater velocities. This allows for a shorter lifetime requirement for the spacecraft, reducing the amount of redundancy and robustness required for the system as a whole. The 1999 NASA study and the KISS study, discussed earlier in the paper, both set goals of reaching this 100 AU distance in less than 10-15 years[21]. Both studies reached the conclusion that a propulsive Oberth maneuver at a low perihelion would maximize heliocentric 
velocities and meet the travel time requirement. Several of these trajectories include optional flyby to further increase heliocentric velocities. While these trajectories would be ideal for the spacecraft examined in this paper, the trajectory would ease lifetime and attitude control requirements. These missions certainly remain as an option, however, alternative trajectories will be examined to determine a reasonable worst case trajectory to allow for a design that can accommodate a variety of trajectories, thus maximizing ridesharing opportunities.

As previously stated, solar system escape velocities require large amounts of energy. Due to the limited mass and volume of the small spacecraft, it cannot rely on its own propulsion system to reach these velocities. While planetary rendezvous missions appear ideal to employ a rideshare architecture, the orbits do not provide enough energy to reach solar system escape velocities. These orbital trajectories are specifically designed to reduce the flight path angle and relative velocity between the planet and spacecraft at arrival, in order to minimize the propulsive maneuver, and thus required propellant, for orbital insertion. This minimizes the effect of gravitational assist maneuvers and does not provide enough of an increase in velocity to escape the Sun's gravity. Therefore, planetary rendezvous missions are not viable solutions for reaching solar system escape velocities. This does limit the available orbital trajectory solutions as planetary rendezvous missions are much more common than interstellar probe missions, however, this is a limitation that can only be overcome with a large propulsive maneuver by the small interstellar probe, negating the minimal mass and volume impingement of the secondary payload on the primary spacecraft.

The resulting orbital trajectory solutions include missions that already plan on reaching solar system escape velocities, such as the Voyager, Pioneer, and New Horizons missions. However, the interstellar missions discussed by NASA in 1999 and KISS may also host this spacecraft in an effort to reach multiple destinations with 
one mission. The spacecraft concepts discussed in those studies were roughly 600-700 $\mathrm{kg}$, meaning the secondary payload would increase the mass of the primary interstellar probe by approximately 5\%. Regardless, the spacecraft would need to join a mission that is already exiting the solar system. Although the spacecraft is ideal as a secondary payload, a primary mission could be designed to bring 5-10 of these probes. The carrier spacecraft would embark on its solar system escape trajectory and drop the probes off at different times in the mission. These probes could then spread out, returning data to provide a global view of this $100 \mathrm{AU}$ distance over a wide swath of the OSS.

The main benefit of this spacecraft is that it is meant to return data at a different location than the primary spacecraft, multiplying the scientific data yield of an interstellar mission. However, because the spacecraft cannot rely on a large propulsive maneuver to reach this alternate destination, a different method of diverting off the original trajectory must be determined. This can be easily realized with the use of a gravitational assist maneuver, which is conveniently part of many orbital trajectory solutions for interstellar missions. If the secondary spacecraft can impart a small delta-V prior to the flyby, this can change the flyby periapsis, thus changing the resulting trajectory of the spacecraft. This small change in flyby periapsis can result in large changes in the final 100 AU destination.

Analysis was performed on the Voyager 1 and New Horizons trajectory to observe the effect of this change in flyby periapsis. A small delta-V maneuver of $10 \mathrm{~m} / \mathrm{s}$ along velocity vector direction was performed at different points before the ultimate flyby maneuver of the mission, Jupiter for New Horizons and Saturn for Voyager 1. Orbital elements for the Jupiter to Saturn leg of the Voyager 1 trajectory and the Earth to Jupiter leg of the New Horizons trajectory were taken from JPL's Horizons tool[16]. The trajectories were calculated as 2 dimensional flyby maneuvers and treated as instantaneous changes of direction and velocity after the flyby. 


\subsection{Voyager 1 Flyby Sensitivity}

Voyager 1 performed two flybys, one at Jupiter followed by a Saturn flyby. This provided significant added velocity to propel it to the current speed of 3.6 AU/year. Sensitivity analysis was performed to analyze the effects of a small delta-V maneuver before the flyby to change the resulting destination. For the Voyager 1 trajectory, it was assumed that the secondary spacecraft would be deployed from the host spacecraft shortly after the Jupiter flyby. To analyze the effect of the maneuver, the delta-V was applied at different times during the Jupiter to Saturn leg of the trajectory. The orbit was the propagated until it reached the radial distance of 9.6 AU, Saturn's average distance from the Sun. The resulting angle change from the nominal flyby was calculated, in addition to the arc length change. This change in arc length distance was assumed to be the change in flyby periapsis, which would cause the change in resulting trajectory. Due to the very short changes in arc length on the order of several planetary radii, the arc length change should essentially be equivalent to a linear distance change, as the curve in the arc length change is essentially negligible. The assumption also negates the change in distance of the planet due to different arrival times as a result of the burn maneuver. However, taking this into account, the flyby periapsis should be further affected. This is because a burn in the velocity vector direction would result in the spacecraft reaching the intersection point of the planet earlier, and the planet would not have progressed as far in its orbit. Because the flyby will need to occur at the trailing end of the planet's orbit, this means the flyby periapsis would be further decreased with a delta-V applied in the velocity vector direction. This also applies to the opposite case in which delta- $\mathrm{V}$ is applied in the opposite direction of the velocity vector, resulting in an increased flyby periapsis due to the spacecraft arriving at the intersection point later in time. The orbit was propagated in 2 dimensions and no orbital perturbations were included. These effects 
should be investigated in future studies. The analysis produced results detailed in Figure 5.1. The closest flyby altitude was assumed to be 4 Saturn radii, or 5 Saturn radii in flyby periapsis. For reference, Voyager 1 flew by Saturn at a flyby periapsis of roughly over 3 Saturn radii.
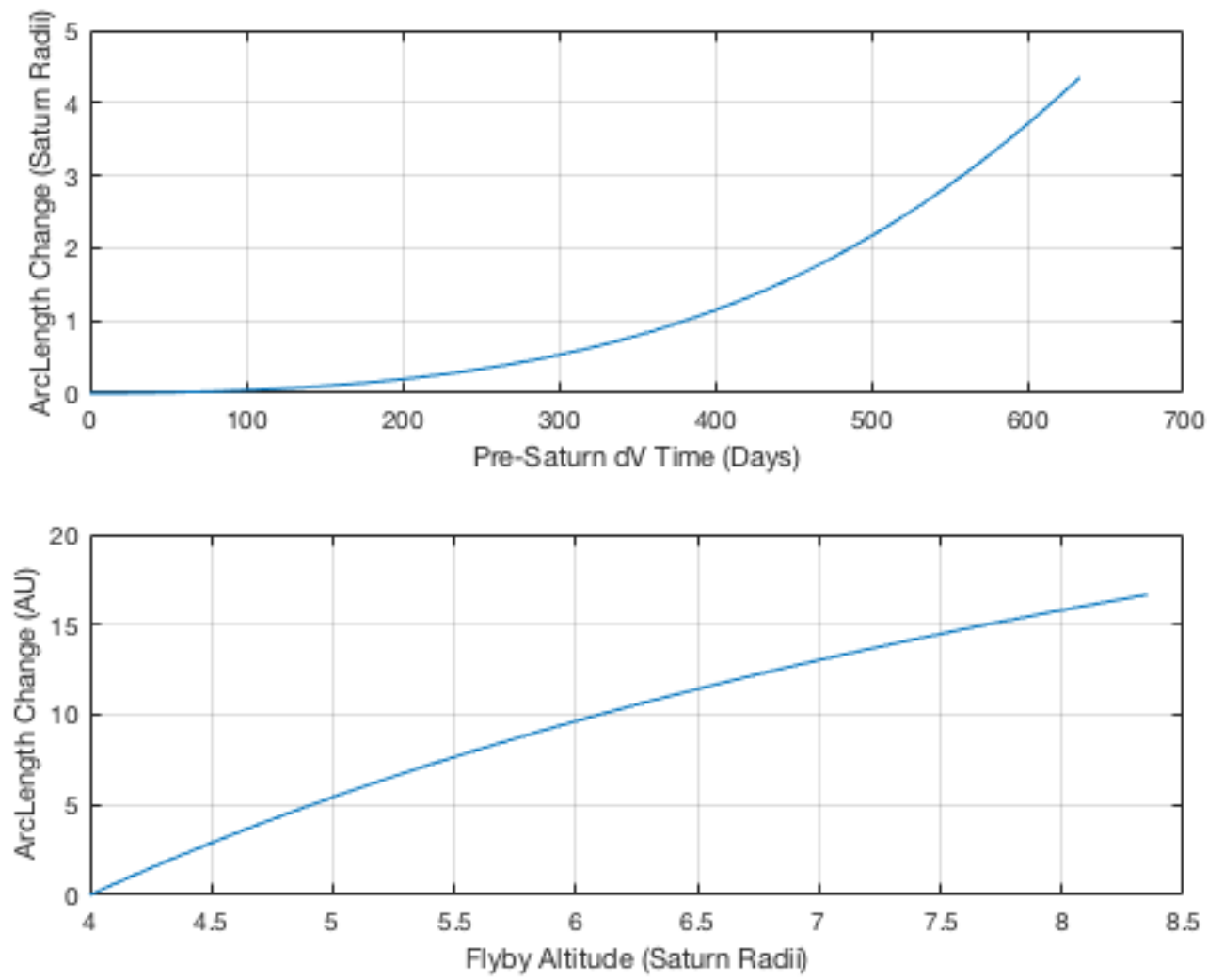

Figure 5.1: Trajectory Effects of Small Delta-V Maneuver Prior to Saturn Flyby

Figure 5.1 details the arc length change as a function of the delta-V maneuver performed at different times before the Saturn flyby. The trend shows that the earlier the delta- $\mathrm{V}$ maneuver was performed, the larger the change in the final position when the spacecraft reaches 9.6 AU. The spacecraft can alter its flyby periapsis over 4 Saturn radii if the propulsive maneuver is performed shortly after the Jupiter flyby. These differences in Saturn intercept positions were then used to analyze the differences in resulting trajectory with different flyby periapses. The orbit was then propagated 
until the spacecraft reached 100 AU in radial distance from the Sun. The lower graph in Figure 5.1 details the change in arc length of the final $100 \mathrm{AU}$ position. This trend shows that if the $10 \mathrm{~m} / \mathrm{s}$ delta- $\mathrm{V}$ maneuver is performed early enough, the secondary spacecraft can reach a final position over $15 \mathrm{AU}$ in arc length away from the primary spacecraft's trajectory. A visual of the largest difference is shown in Figure 5.2. This plot shows the differences in trajectory when the spacecraft performs its maneuver at its earliest opportunity.

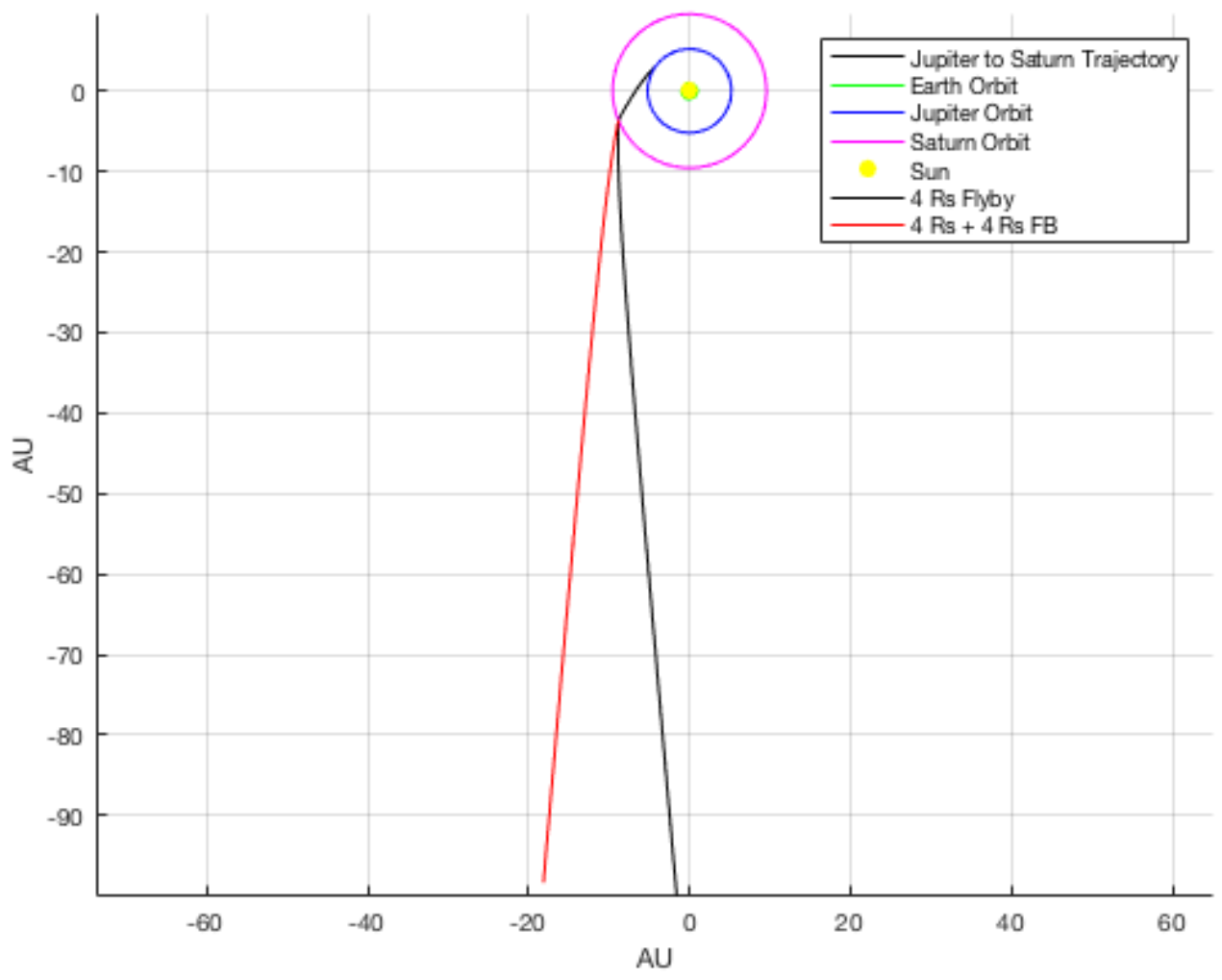

Figure 5.2: Visual of Trajectories as a Result of Saturn Flyby Sensitivity Analysis

The delta- $V$ maneuver in the velocity vector direction would cause the spacecraft to reach Saturn earlier, therefore, the closest flyby of 4 Saturn radii would occur when the spacecraft performed the delta- $\mathrm{V}$ maneuver at its earliest opportunity after 
encountering Jupiter. It is anticipated that similar results would occur if the spacecraft performed the delta- $\mathrm{V}$ maneuver in the opposite direction, slowing itself down, however, this burn is not ideal due to the secondary spacecraft's configuration. Nevertheless, this shows that if one of these secondary spacecraft was included on the Voyager 1 mission, it would have the ability to reach a drastically different final 100 AU position with this small burn.

\subsection{New Horizons Flyby Sensitivity}

Similar analysis was performed on the New Horizons mission. This analyzed the effect of the delta-V maneuver performed along the Earth to Jupiter leg of the mission and its resulting effects on the Jupiter flyby and final 100 AU position. Once again, the delta- $\mathrm{V}$ maneuver was assumed to be $10 \mathrm{~m} / \mathrm{s}$ in the velocity vector direction. The analysis produced the following trends detailed in Figure 5.3 

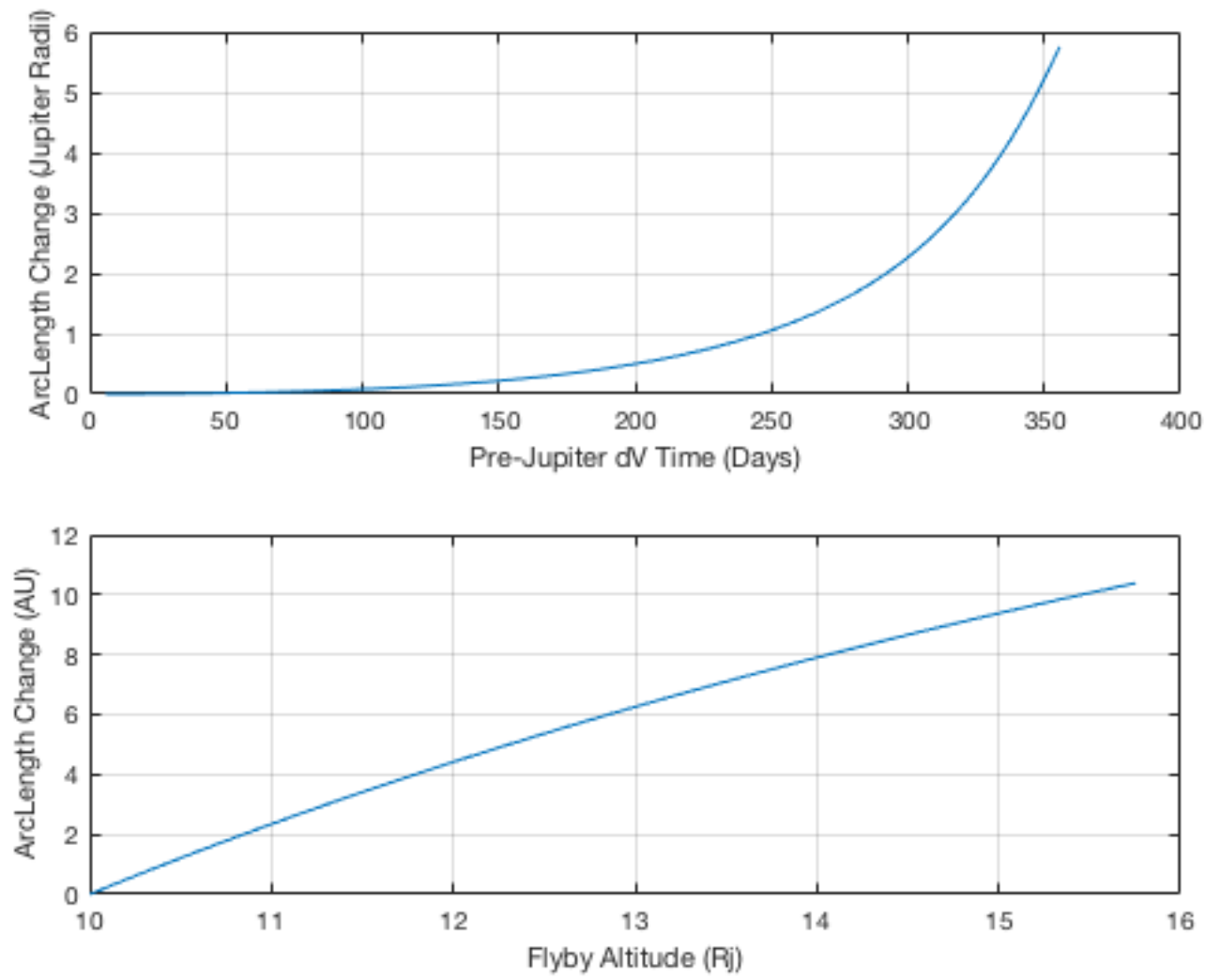

Figure 5.3: Trajectory Effects of Small Delta-V Maneuver Prior to Jupiter Flyby

Similar to the Voyager 1 case, the earlier the maneuver is performed, the larger the difference is in position at the Jupiter encounter. This analysis used a similar method, propagating the orbit until the spacecraft reached Jupiter's average distance to the Sun of around 5.2 AU. The nominal flyby altitude in this case was chosen to be 10 Jupiter radii, as the radiation belts at Jupiter are extremely harsh. This requires the spacecraft to perform the flyby at further distances to Jupiter to incur less radiation damage. The small maneuver was able to change the intercept position at Jupiter by over 5 planetary radii. This resulted in over $10 \mathrm{AU}$ in arc length change at the final $100 \mathrm{AU}$ position. Figure 5.4 shows a visual of the largest change as a result of the small propulsive maneuver. 


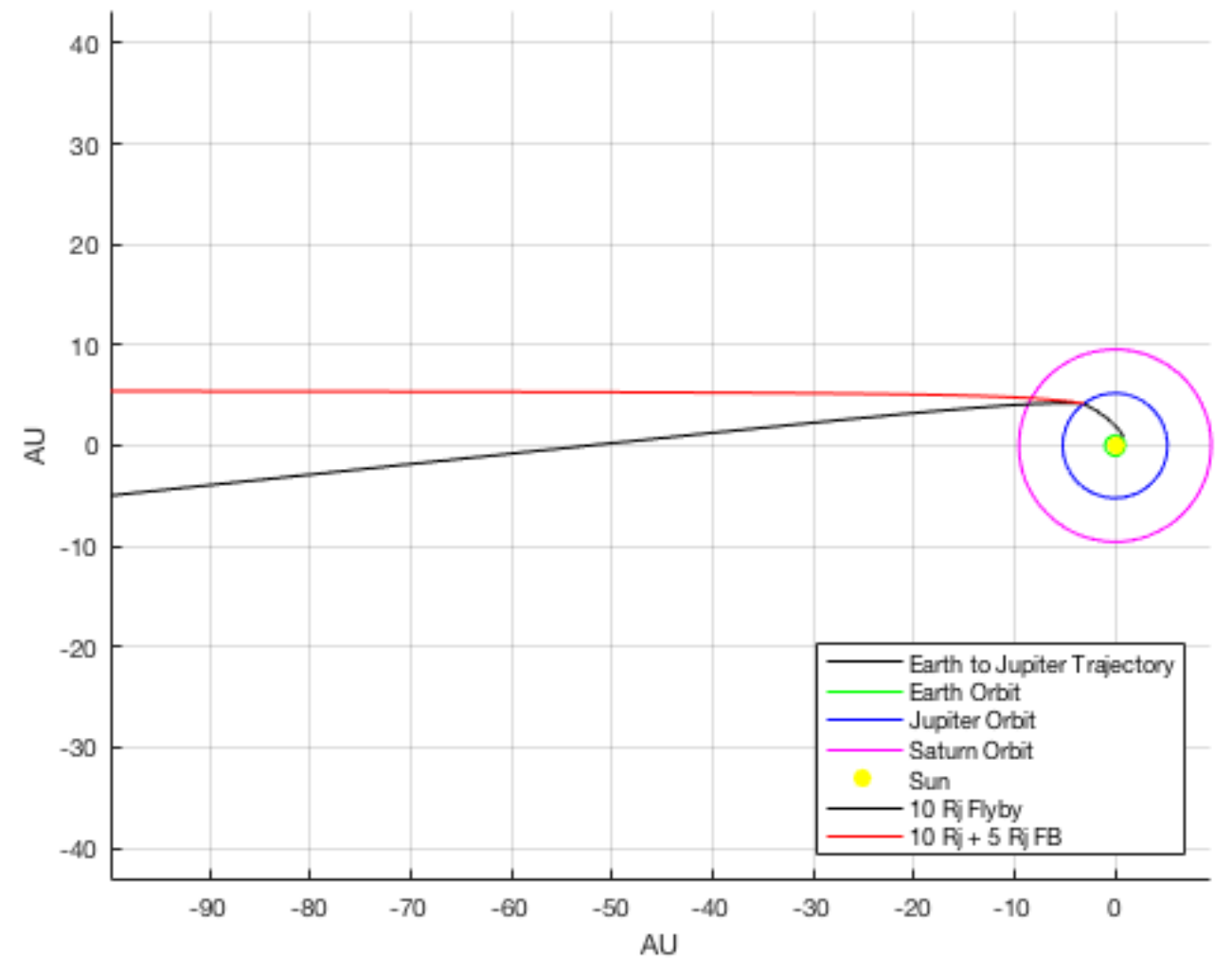

Figure 5.4: Visual of Trajectories as a Result of Jupiter Flyby Sensitivity Analysis

\subsection{Swarm Architecture Possibilities}

The results of this sensitivity analysis shows that large changes in final 100 AU positions can be achieved with a small propulsive maneuver before the planetary encounter. This shows promise that a mission employing a swarm architecture may be able to map out large portions of the OSS environment. If a host spacecraft were to house multiple of these small interstellar probes, they could be deployed during various portions of the pre-flyby leg of the trajectory and perform the delta- $\mathrm{V}$ maneuver so that each spacecraft encounters the planet at different flyby periapsiss. This would allow the swarm of probes to spread out and cover tens of AU of space with very small delta-V maneuvers, providing a truly global view of the deep space 
environment. These maneuvers could be designed to even change the inclination at which the flyby was performed, allowing the probes to cover portions of deep space out of the ecliptic plane. However, it should be noted that maneuvers intended to change the inclination of the flyby would have smaller effects with similar delta- $\mathrm{V}$ values as impulsive maneuvers are most effective in the velocity vector direction. On the other hand, the spacecraft would not need a large change in z-axis to enter a high inclination hyperbolic orbit about the planet.

\subsection{Viable Orbital Trajectories}

As mentioned earlier in this section, the viable trajectories are those that are already leaving the Solar System. These orbital solutions can be similar to the Voyager, Pioneer and New Horizons missions, or the spacecraft may join a flagship interstellar mission as thought up in the 1999 NASA study or the KISS study. However, several considerations may narrow down the available solutions. Of the Voyager 1 and New Horizons trajectories, the Voyager 1-like trajectory (Jupiter to Saturn) is a better option for the small OSS probe mission. Jupiter's radiation belts are severely hazardous and pose a threat to the electronics of this small spacecraft. While Voyager 1 also passed by Jupiter, the small OSS probe may stay housed inside the host spacecraft, protected by more shielding that would otherwise be unavailable on the small probe itself, citing mass and volume constraints. Furthermore, the small probe's electronics could be shut down during the flyby to lessen the radiation damage. Once clear of Jupiter's radiation belts, the probe could turn on, eject from the host spacecraft, perform the delta- $\mathrm{V}$ maneuver and simply coast toward Saturn, performing its unprotected flyby through much weaker radiation belts. However, it should be noted that the launch windows for Earth-Jupiter missions occur nearly every year, rather than the roughly 20 year gap in launch windows for a Voyager 1 style trajectory[39]. 
Nevertheless, the next Jupiter-Saturn alignment after 2020 will occur in 2040, leaving 10 years for technological advancements and another 10 years for spacecraft and mission design.

Another significant concern for choosing a suitable trajectory for the spacecraft is that ejecting close to the Sun presents a large thermal problem. With the spacecraft deployable reflector dish acting as a solar concentrator, the spacecraft will receive significantly more thermal energy from the sunlight when close to the Sun, as the dish is sized for its $100 \mathrm{AU}$ destination. For example, at Jupiter, the spacecraft would receive 400 times the thermal energy that it would normally see at 100 AU. Therefore, designing the spacecraft to operate even closer than Jupiter will pose a significant thermal challenge. This will be discussed further in the next chapter. However, this means New Horizons-like trajectories will be a less attractive option, as the spacecraft will need to survive distances from 1 to $100 \mathrm{AU}$, if it is to perform the delta$\mathrm{V}$ maneuver along the Earth-Jupiter leg and change its flyby periapsis at Jupiter. Primary interstellar probe missions are also viable trajectory solutions, such as the close perihelion Oberth maneuver trajectory, however, similar thermal considerations should be kept in mind and a flyby will likely be required to allow the probe to reach a significantly different destination from the primary spacecraft. The thermal aspect of the probe will be examined further in the Power and Thermal Subsystem chapter.

For these reasons, the Voyager 1-like trajectory beginning at Jupiter will selected as a reference mission and analyzed to determine the requirements and required subsystems. This orbital trajectory case will provide a baseline for subsystem requirements and allow for reasonable solutions that meet the system requirements. 


\section{Chapter 6}

\section{DEPLOYABLE ANTENNA SUBSYSTEM}

The deployable subsystem is largely the most important aspect of the spacecraft. Because the spacecraft must generate power with a non-nuclear power source, the only other option is solar power at these large distances. In addition, the small size of the spacecraft requires a large deployable system to capture ample amounts of energy. This system must pack into a small enough volume, as well as remain relatively low mass.

As described in previous sections, deployable systems are becoming attractive options for small spacecraft due to their limited volume and mass. These deployable systems offer improved performance while maintaining relatively light in mass and high in packing efficiency. A deployable system would be necessary for this spacecraft to perform two key functions: 1) concentrate enough solar energy for power generation and 2) provide a high gain antenna for communications back to Earth. The general concept of the multi-purpose deployable antenna is shown in Figure 6.1. This configuration uses an inflatable antenna system based on an existing model, which will be described later in this section. However, the figure details the general functions of this reflector antenna concept. The reflector will be deployed from a small volume into a parabolic shape with a focal point resting somewhere inside the bus. This reflector will then concentrate sunlight onto solar panels along the inner surface, collecting large amounts of low intensity sunlight. A feed placed on the same surface as the solar cells will bounce RF waves off the large reflector dish to communicate with Earth. The reflector surface will need to be a material that is both reflective for $\mathrm{RF}$ and the visible light spectrum. 


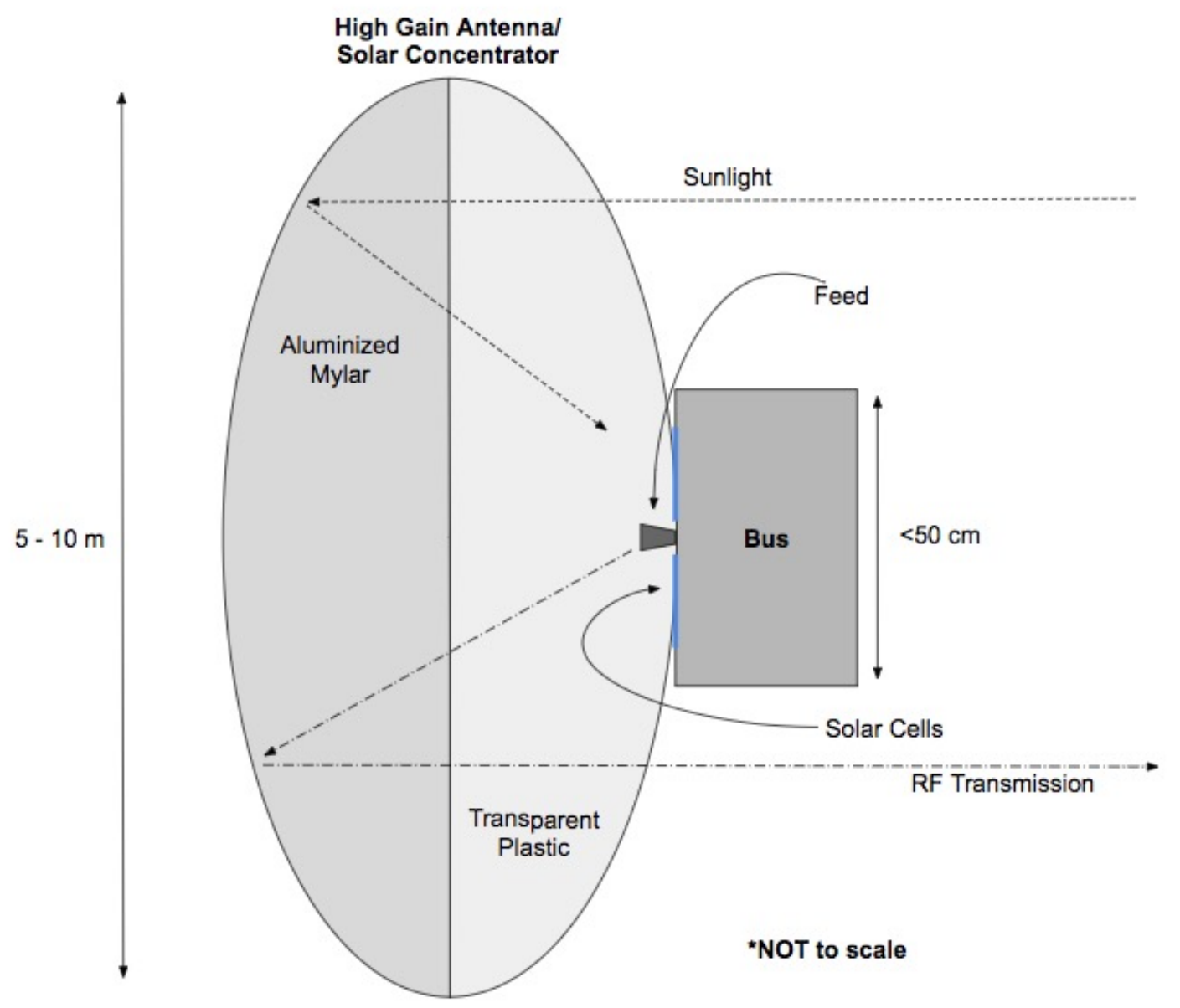

Figure 6.1: Deployable Reflector Concept Showing Power Generation and High-Gain Antenna Functions

To determine an adequate deployable reflector system for the spacecraft, we must first examine the available systems and predict future capabilities. Currently, many solar sail and reflector antenna systems are available and many more are under development. Observing available technologies will allow for a reasonable estimate for a realistic deployable system for the spacecraft that is both low mass and can pack efficiently into a small volume. 


\subsection{Existing Deployable Technologies}

Existing deployable technology has been examined to determine an adequate system for the OSS probe. Table 6.1 details some of the systems that have been flown or are currently under development.

Table 6.1: Existing Deployable Technologies

\begin{tabular}{|c|c|c|c|c|}
\hline \multicolumn{5}{|c|}{ Reflector Antennas } \\
\hline Name (Organization) & Diameter $(\mathrm{m})$ & Area $\left(\mathrm{m}^{2}\right)$ & Volume (U) & Packing Efficiency $\left(\mathrm{m}^{2} / \mathrm{U}\right)$ \\
\hline RainCube (NASA-JPL)[40] & 0.80 & 0.50 & 2.00 & 0.25 \\
\hline KaTENna (Tendeg) $[12]$ & 1.00 & 0.79 & 3.00 & 0.26 \\
\hline KaPDA (NASA-JPL)[41] & 0.80 & 0.50 & 1.50 & 0.33 \\
\hline Mini Deployable High Gain Antenna (Boeing)[42] & 0.50 & 0.20 & 0.40 & 0.50 \\
\hline X-Band Inflatable Antenna (NASA-JPL) [3] & 1.00 & 0.79 & 0.50 & 1.57 \\
\hline 6U Antenna (Physical Sciences Inc.) [43] & 1.95 & 3.00 & 2.00 & 1.50 \\
\hline P-DaHGR (MMA Design LLC.)[44] & $0.80-5.00$ & $1-20$ & $2-40$ & 0.50 \\
\hline T-DaHGR (MMA Design LLC.)[45] & $0.80-5.60$ & $1-25$ & $1-25$ & $0.50-1.00$ \\
\hline \multicolumn{5}{|c|}{ Drag/Solar Sails } \\
\hline Name (Organization) & Diameter $(\mathrm{m})$ & Area $\left(\mathrm{m}^{2}\right)$ & Volume (U) & Packing Efficiency $\left(\mathrm{m}^{2} / \mathrm{U}\right)$ \\
\hline FREEDOM (Tohoku University)[46] & 1.38 & 1.50 & 1.00 & 1.50 \\
\hline NanoSail-D2 (NASA-MSFC)[47] & 3.57 & 10.00 & 3.00 & 3.33 \\
\hline dragNET (MMA Design LLC.)[48] & 4.22 & 14.00 & 3.70 & 3.78 \\
\hline Deorbit Sail (Surrey Space Center)[49] & 4.51 & 16.00 & 3.00 & 5.33 \\
\hline Cubesail (Surrey Space Center)[50] & 5.00 & 25.00 & 2.00 & 12.50 \\
\hline LightSail (Planetary Society)[32] & 6.38 & 32.00 & 2.00 & 16.00 \\
\hline NEAScout (NASA-MSFC)[24] & 10.46 & 86.00 & 2.00 & 43.00 \\
\hline
\end{tabular}

Because the spacecraft will be relying on near-term improvements in deployable technologies, it is important to understand the current capabilities of these types of systems.

\subsubsection{Drag/Solar Sails}

Solar sails, in theory, are a popular method of efficient navigation through space, although, they have seldom been used on spacecraft. These systems offer propellant- 
less propulsion by deploying large reflective sails that rely on solar radiation pressure for impulse. Although the method requires active attitude control, it can provide significant delta- $\mathrm{V}$ without propellant, given the spacecraft has a low area-to-mass ratio. To achieve these low area-to-mass ratios, deploying a large sail with low mass is desirable. Drag sails follow the same principles in that they must achieve low areato-mass ratios to maximize performance. The difference is that drag sails receive accelerations due to the drag force from the thin atmosphere in low altitude orbits. Technological advancements in extremely thin materials as well as packing and folding techniques is constantly improving the packing efficiencies of these drag and solar sail systems.

To roughly determine the capabilities of these technologies in 10-15 years time, we can examine current and past systems and generate a basic trend line. Figure 6.2 shows a variety of packing efficiencies in area per CubeSat standard unit volume, U, for current and past systems plotted according to the year of expected flight readiness. The figure also include a linear regression line predicting where this technology might end up by the year 2030. Solar and drag sails show a clear increase in packing efficiencies over the years, indicating that this trend will continue as technology advances. The trend line roughly estimates that solar sail technology should be able to reach packing efficiencies of over $30 \mathrm{~m}^{2} / \mathrm{U}$ by 2030 . It should be noted that the trend line is likely not linear and would probably resemble a logarithmic curve as a function of material limitation. Nonetheless, the function of Figure 6.2 is to show that packing efficiencies of this technology is improving over time. 


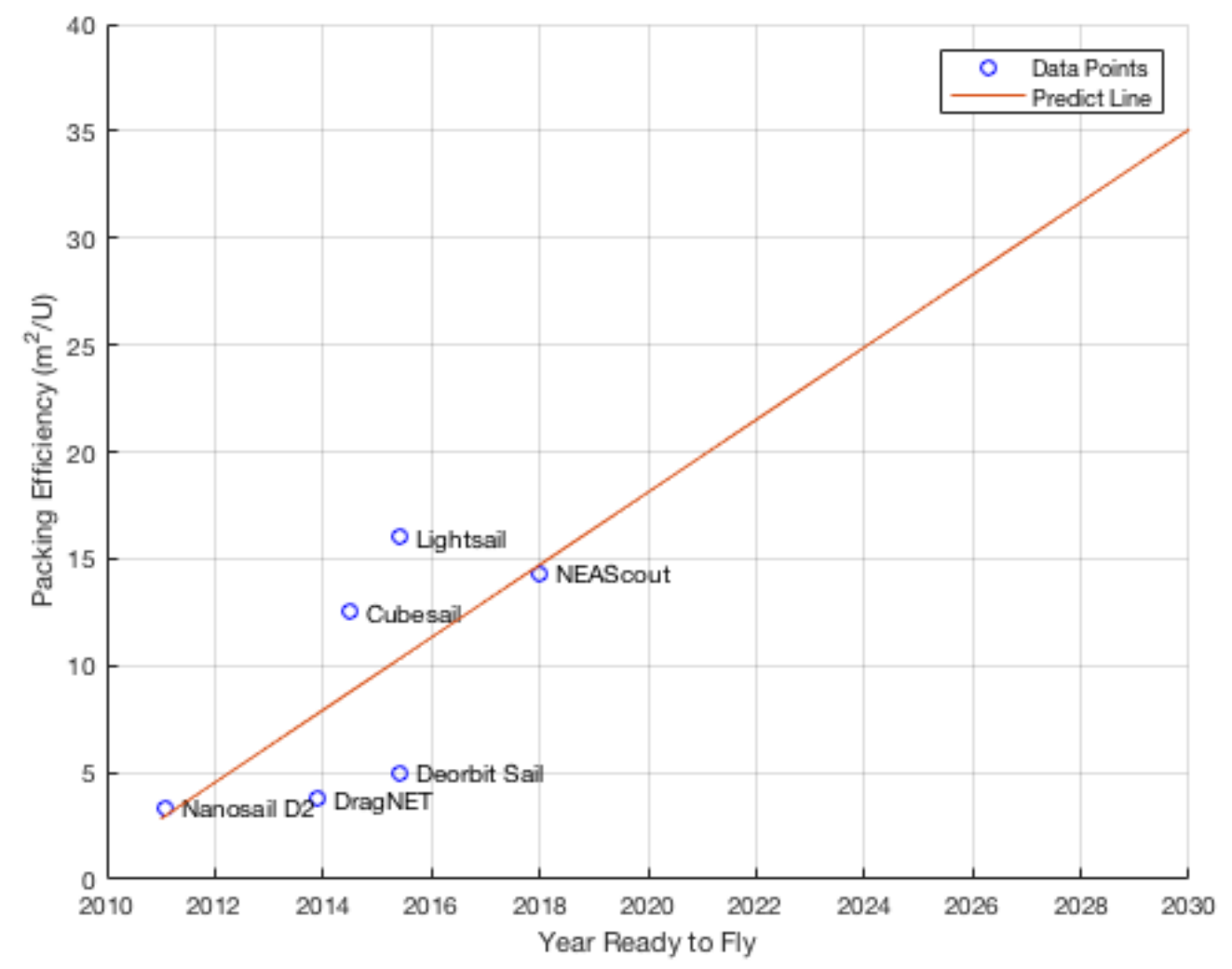

\section{Figure 6.2: Packing Efficiency Trending of Solar and Drag Sails}

\subsubsection{Deployable Reflector Antennas}

Deployable reflectors are also popular among small spacecraft, allowing these nanosatellites to compete in communications performance with larger and more traditional spacecraft, at a significantly lower cost. These deployable systems will be much more useful to the proposed solar-powered OSS probe, as the antennas can be of parabolic shape, which is conducive to the solar concentrator function of the deployable system. While the configuration of these antennas are desirable, they often have lower packing efficiencies, due to the more specific shape requirements and the need to position the antenna away from the main body of the spacecraft. These deployable antennas usually are not planar like solar and drag sails, resulting in more complex systems in 
general. Although some antennas can be planar, such as a phased array antennas, these are inadequate shapes for the solar concentrator function of the deployable reflector system.

Less development has occurred in small spacecraft deployable antenna technology, however, interest is growing in this region. Because nano-satellites are becoming interplanetary, such as the MarCO CubeSats that have already been launched towards Mars, the need for deployable antenna systems to allow these small spacecraft to communicate with Earth over these large distances will grow. Similar to the solar and drag sail systems, observing the packing efficiencies over time can give a rough idea of what packing efficiencies may be available in the near future. However, because there are less deployable antenna systems currently available and under development, there will be larger uncertainties in terms of the observed packing efficiency trend. In addition, there are several systems designed for high data rates that result in low packing efficiencies. For example, RainCube is a technology demonstration to profile precipitation on Earth using a Ka-Band deployable antenna. However, because the surface accuracy requirements for Ka-Band are quite challenging, the resulting deployable antenna design is a 0.5 meter diameter dish unfolding out of a 1.5U compartment[40], which pales in comparison to other deployable antenna systems. Because these systems are not primarily designed with packing efficiency in mind, they do not accurately represent the technological capabilities and, therefore, will not be included in the trend analysis. Nonetheless, several currently available and under-development systems are shown in Figure 6.3. 


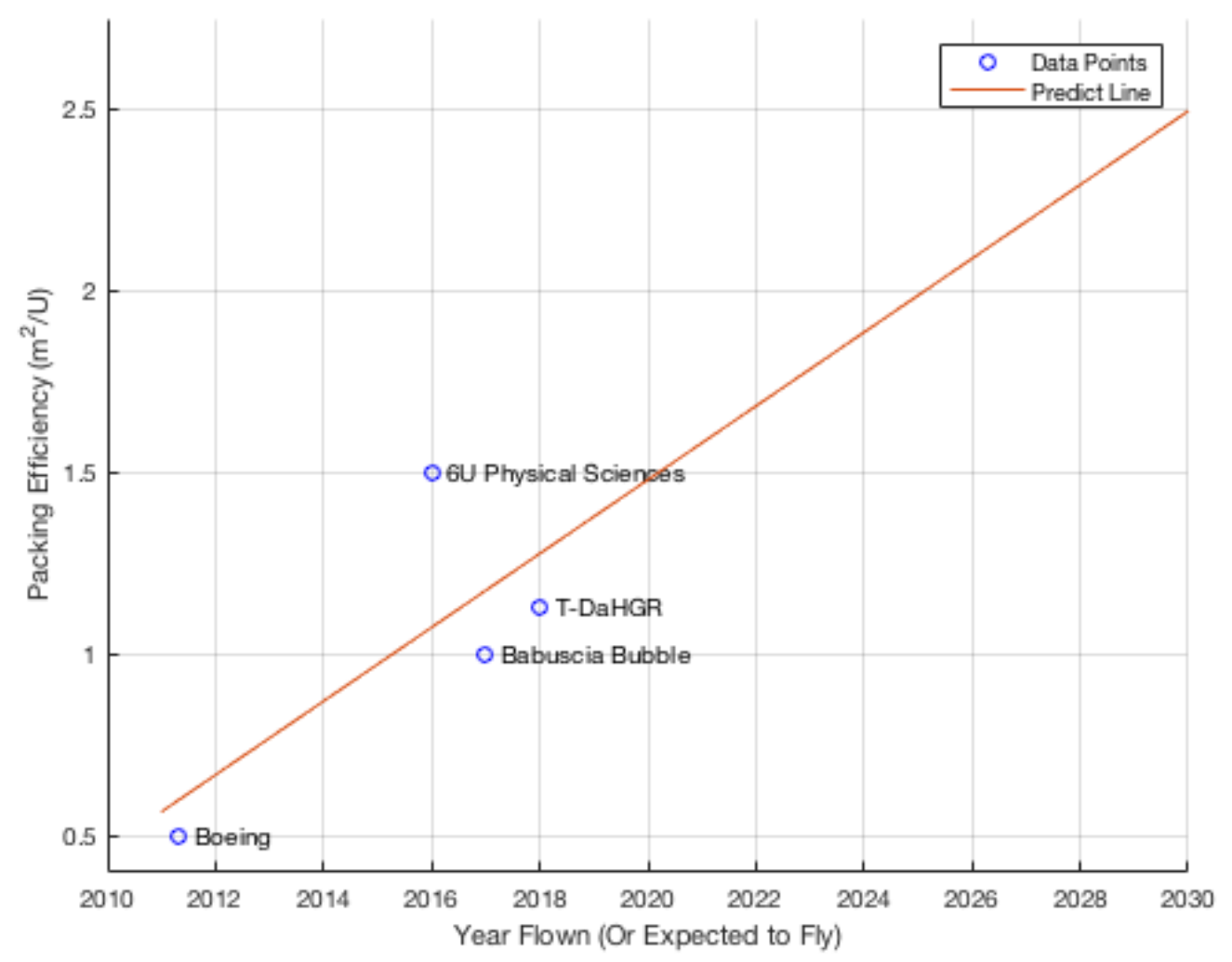

\section{Figure 6.3: Packing Efficiency Trending of Deployable Antenna Systems}

Although there is a trend line, it consists of very few points. Therefore, the trend could be wildly inaccurate. Nonethelss, deployable reflector technology is relatively new and there is potential for significant advancements in this area of deployable technology.

\subsubsection{Inflatable X-Band Antenna (NASA-JPL)}

One deployable antenna system is ideal for the solar-powered OSS spacecraft design. A group at NASA Jet Propulsion Laboratory headed by Alessandra Babuscia has been developing a deployable CubeSat antenna that works by inflating a bubblelike membrane into a parabolic shape with sublimate material[3]. The antenna is a 
reflector in which a feed reflects $\mathrm{RF}$ waves off a parabolic surface to direct it back towards the target. The design consists of half a metalized Mylar that acts as the reflector surface, and the other half is transparent Mylar. The antenna retains its shape after initial inflation with the use of UV rigidization strips. These strips will maintain the parabolic shape after the pressurized gas inevitably leaks out of the system. While the rigidization process takes two hours with solar intensities at Earth, it should be kept in mind that the rigidization process will take longer if the spacecraft is ejected from the host spacecraft at distances further than 1 AU. For reference, if the spacecraft is ejected at Jupiter, the rigidization process would take roughly 50 hours. However, this problem can be easily solved by carrying more sublimate powder.

The design is ideal for the solar-powered OSS probe due to the combination of the metalized Mylar material choice and the parabolic shape. This will enable the dual functionality of the deployable system as the metalized Mylar is reflective for both RF and visible light waves. While aluminized Mylar degrades due to UV exposure, the dish can be deployed further away from the sun, resulting in reduced degradation rates. However, an alternative material that should be studied and traded against aluminized Mylar is aluminized Kapton, which degrades much less when exposed to UV radiation. In addition, because the antenna is pressurized, surface irregularities are minimized compared to boom deployed systems, resulting in higher solar concentration efficiencies. The choice of RF frequency will be discussed in the Communications Subsystem chapter, however, higher frequencies are more sensitive to surface irregularities such as wrinkles. The dish shape will need to be designed with a focal point that will illuminate the solar cells around the feed, therefore, the feed will not be optimally placed, thus incurring losses in efficiency. The feed placement effects will be discussed further in the dish shape section of this paper. Figure 6.4 shows a picture of the antenna in an anechoic chamber. 


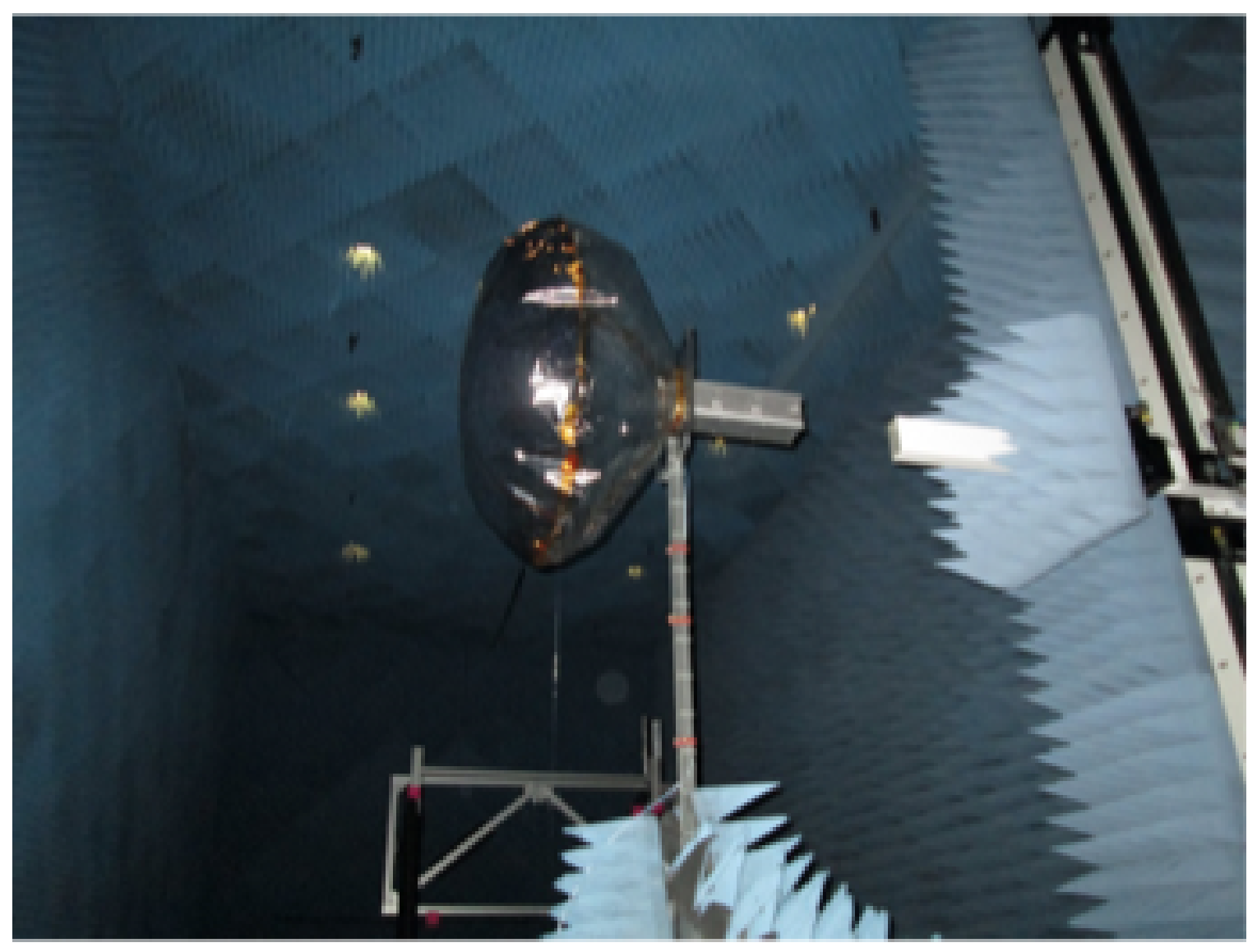

Figure 6.4: NASA JPL Inflatable Antenna in an Anechoic Chamber[8]

Nevertheless, this antenna design fits the mission requirements well. The dish is ultra-compact and fits a $1 \mathrm{~m}$ diameter dish in a $0.5 \mathrm{U}$ volume and weighs $0.5 \mathrm{~kg}$. New iterations of the design has modified the antenna to a spherical shape and a smaller diameter of $0.7 \mathrm{~m}[51]$. However, for the purposes of this paper, it will be assumed that a parabolic shaped inflatable antenna of the original is feasible. Babuscia states that the design is easily scalable to much larger sizes. Table 6.2 details the expected mass and volume required for larger diameter antenna designs[3]. The resulting spacecraft will a dish much larger than $1 \mathrm{~m}$ to capture enough sunlight at $100 \mathrm{AU}$, however, the scalability of this design is unparalleled to traditional deployable dish systems and shows promise that it can satisfy the overarching system mass and dimension requirements mentioned in the previous chapter. 


\section{Table 6.2: Inflatable Antenna Scalability in Size[3]}

\begin{tabular}{|c|c|c|}
\hline Diameter $(\mathrm{m})$ & Mass $(\mathrm{kg})$ & Volume $(\mathrm{U})$ \\
\hline 1 & 0.4 & 0.4 \\
\hline 2 & 1.4 & 1.4 \\
\hline 4 & 5.1 & 5.1 \\
\hline 8 & 20.5 & 20.5 \\
\hline
\end{tabular}

While the scalability appears promising, the deployable reflector system will need to be at least $5 \mathrm{~m}$ in diameter, which results in approximately $7 \mathrm{~kg}$ and $7 \mathrm{U}$ of volume. If larger sizes are required, the resulting system may be undesirably large in mass and volume. However, assuming packing efficiency trends for these deployable systems, it is reasonable to assume the mass and volume of this system will decrease as advancements in deployable technologies continue. Although the packing efficiency trend in the previous subsection is very rough and based off few points, this paper will assume that packing efficiencies and masses of deployable reflector technology will improve in the near future.

\subsection{Antenna Shape Design}

There is an inherent challenge with designing the shape of the antenna as the focal point of the parabolic dish must be able to illuminate the solar panels without sacrificing the feed efficiency. Ideally the focal point should be placed just behind the feed for maximum dish efficiency when transmitting data. However, the focal point must be placed slightly farther to illuminate the solar panels to generate the necessary electrical power. To determine the necessary shape of the dish, we must calculate the shape of the dish using parabolic shape equations. Figure 6.5 shows the various parameters of a parabola. 


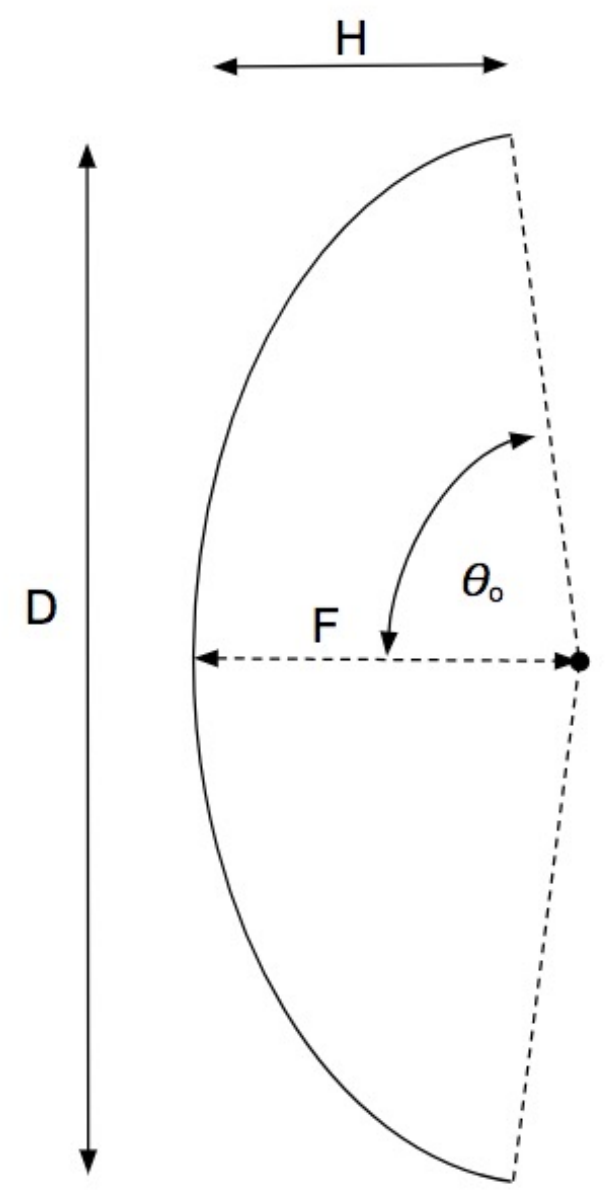

Figure 6.5: Parabola Parameters

These parameters are related through a set of equations that define the shape of the parabola. These can be seen in Equation 6.1 and Equation 6.2[52].

$$
\begin{gathered}
F / D=\frac{1}{4 \tan \left(\frac{\theta_{o}}{2}\right)} \\
F=\frac{D^{2}}{16 H}
\end{gathered}
$$

The focal point problem can be seen in Figure 6.6. Essentially the focal point of the parabolic dish cannot be perfectly placed for the feed, while also generating 
electrical power. This means that the spacecraft must sacrifice dish efficiency in order to generate the necessary electrical power.

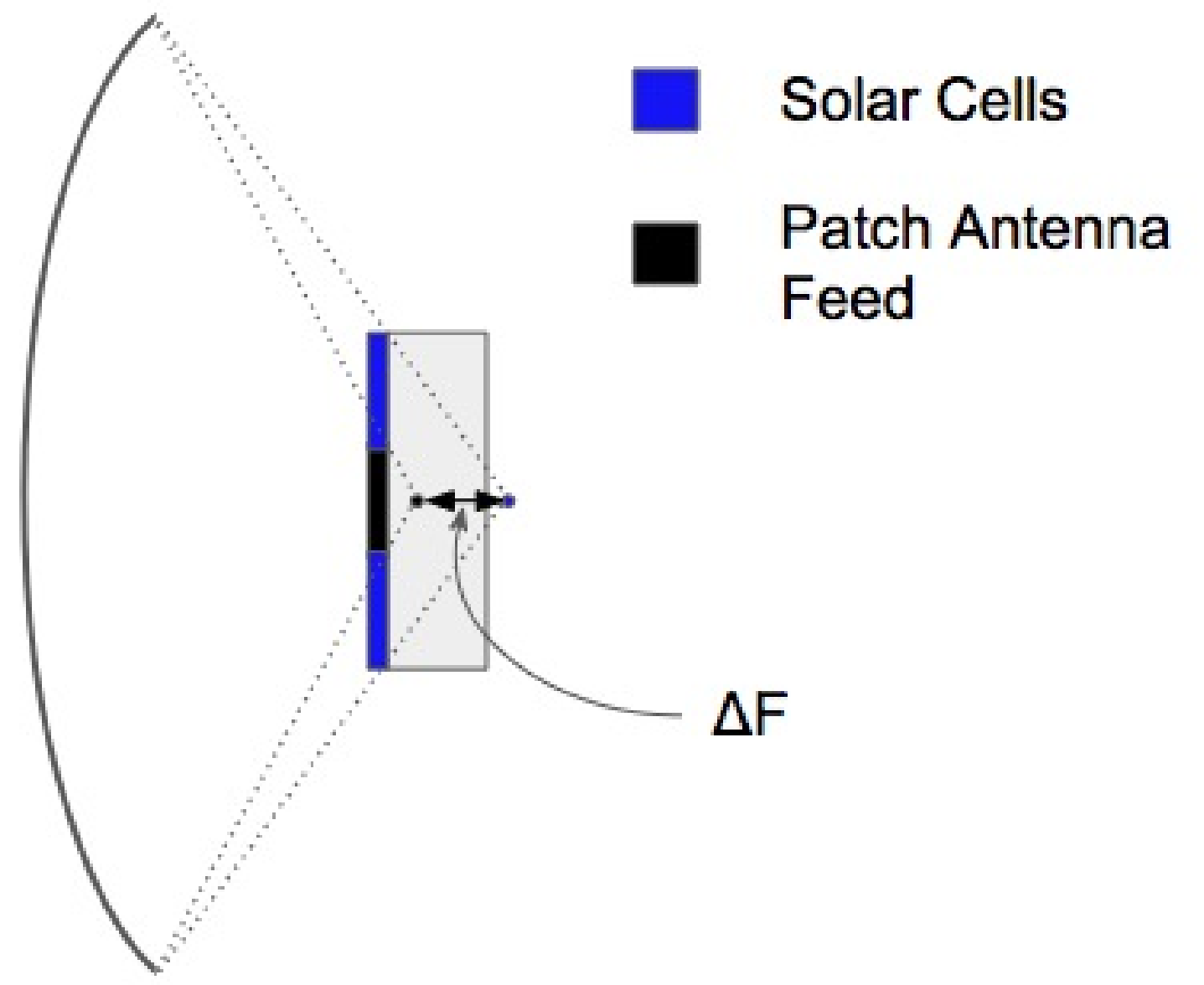

Figure 6.6: The Focal Point Problem

However, through some analysis, the focal point can be placed to minimize dish RF efficiency loss and maximize power generation with the appropriate parabolic dish shape. This efficiency loss is dependent on the wavelength of the RF transmission that the feed is producing. The efficiency curves as a function of the axial displacement of the focal point can be seen in Figure 6.7. 


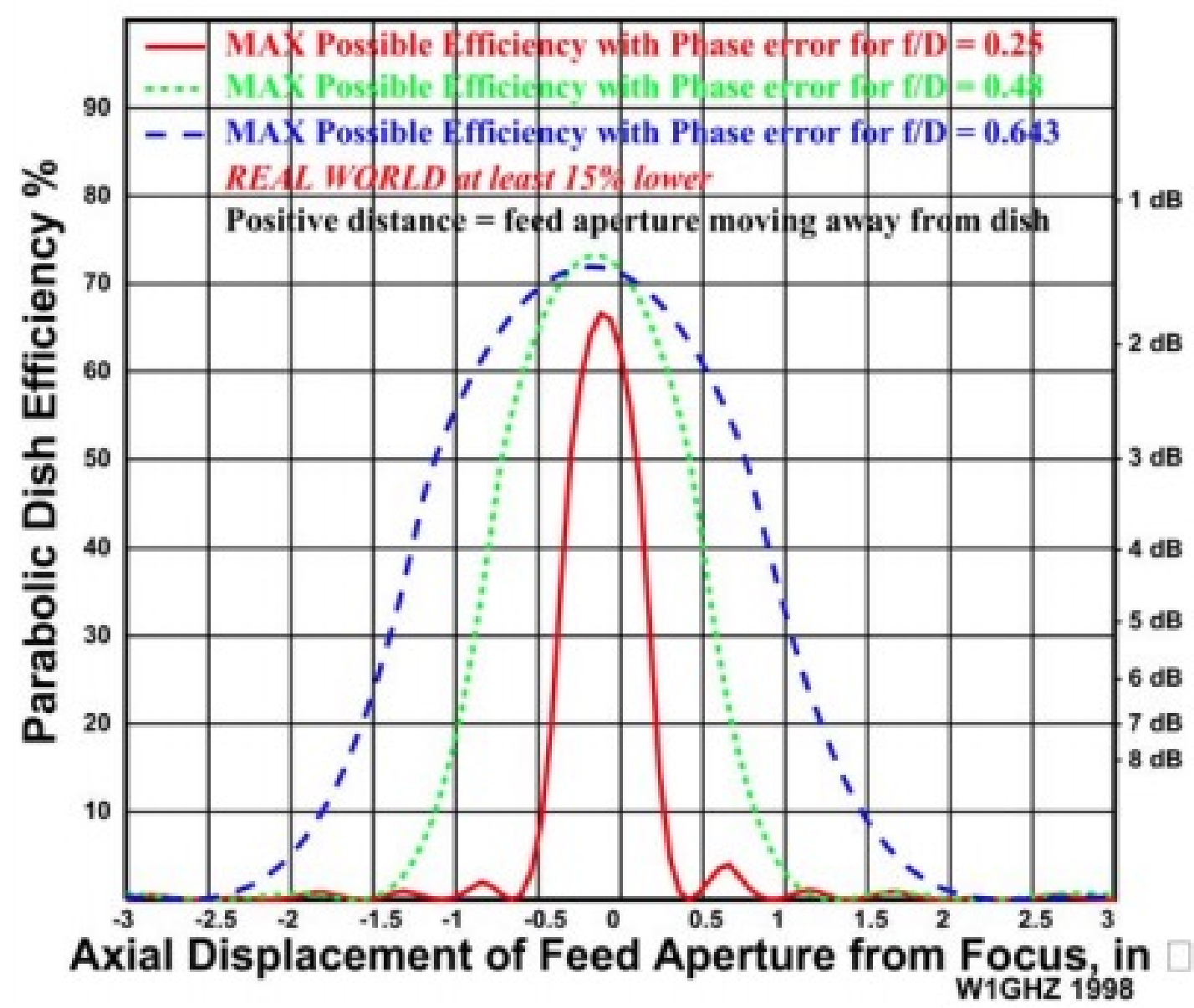

Figure 6.7: Efficiency Loss as a Function of Axial Displacement of the Focal Point from the Feed, courtesy of Paul Wade, W1GHZ[9]

Figure 6.7 shows the parabolic dish efficiency as a function of the axial displacement of the feed aperture from the focal point in wavelengths for a variety of $f / D$ ratios, or the ratio of focal point to diameter of the dish. As the displacement of the focal point from the feed aperture increases, the parabolic dish efficiency decreases. However, the optimal focal point should not be placed on the feed but rather slightly behind the feed so that it covers the aperture of the feed, as is apparent by the shifted curve in Figure 6.7.

The feed will be assumed to be a $10 \mathrm{~cm} \times 10 \mathrm{~cm}$ patch antenna, as is used in Babuscia's design[8]. This will be placed in the center of the panel facing the dish. 
Solar cells will be placed around the feed. The optimal amount of solar cells placed around the feed will be determined through analysis. The solar cell area around the feed can be minimized to minimize the displacement of the focal point from the ideal position, focusing the sunlight onto a smaller area on the face and decreasing the loss of efficiency of the parabolic dish. However, with a constant area occupied by the feed, this decreases the effective area of solar cells that receives sunlight.

Solar panel widths of $2.5 \mathrm{~cm}, 5 \mathrm{~cm}$, and $7.5 \mathrm{~cm}$ on either end of the feed were analyzed. These result in total feed and solar panel widths of $15 \mathrm{~cm}, 20 \mathrm{~cm}$, and $25 \mathrm{~cm}$. Although the patch antenna feed is only $10 \mathrm{~cm}$ wide, it is assumed that the focal point must be placed to cover the diagonals of the feed in order to be optimally placed. Similar considerations must be taken for the solar panel widths. The various cases analyzed can be visualized in Figure 6.8.

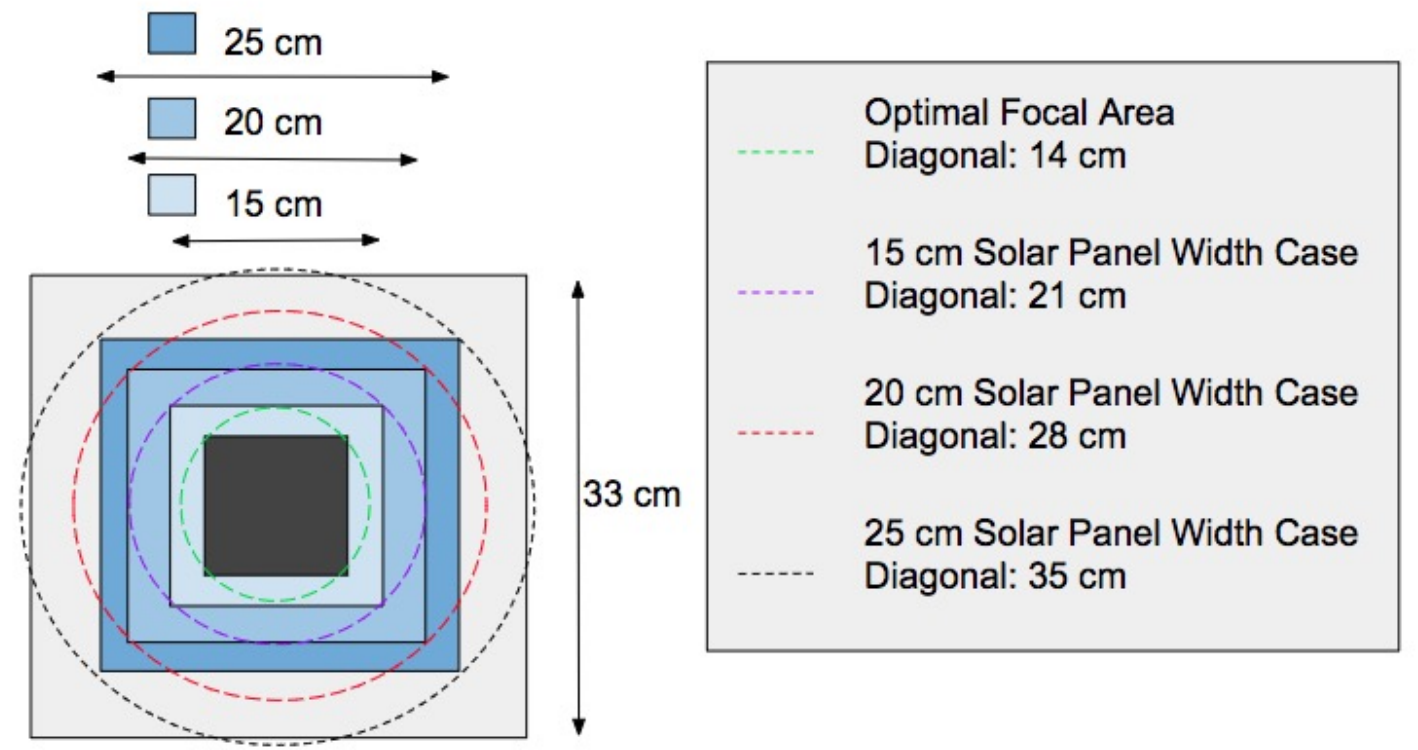

Figure 6.8: Focal Area from Parabolic Concentration for Various Solar Panel Width Cases and Optimal Focal Area

The axial displacement of the focal point from the feed can be calculated as a function of the focal point to diameter ratio of the dish. Using the diagonals of the 
three solar panel width cases and assuming worst case wavelength of $3.57 \mathrm{~cm}$ for $\mathrm{X}$-Band, the axial displacement in wavelengths is calculated in Figure 6.9.

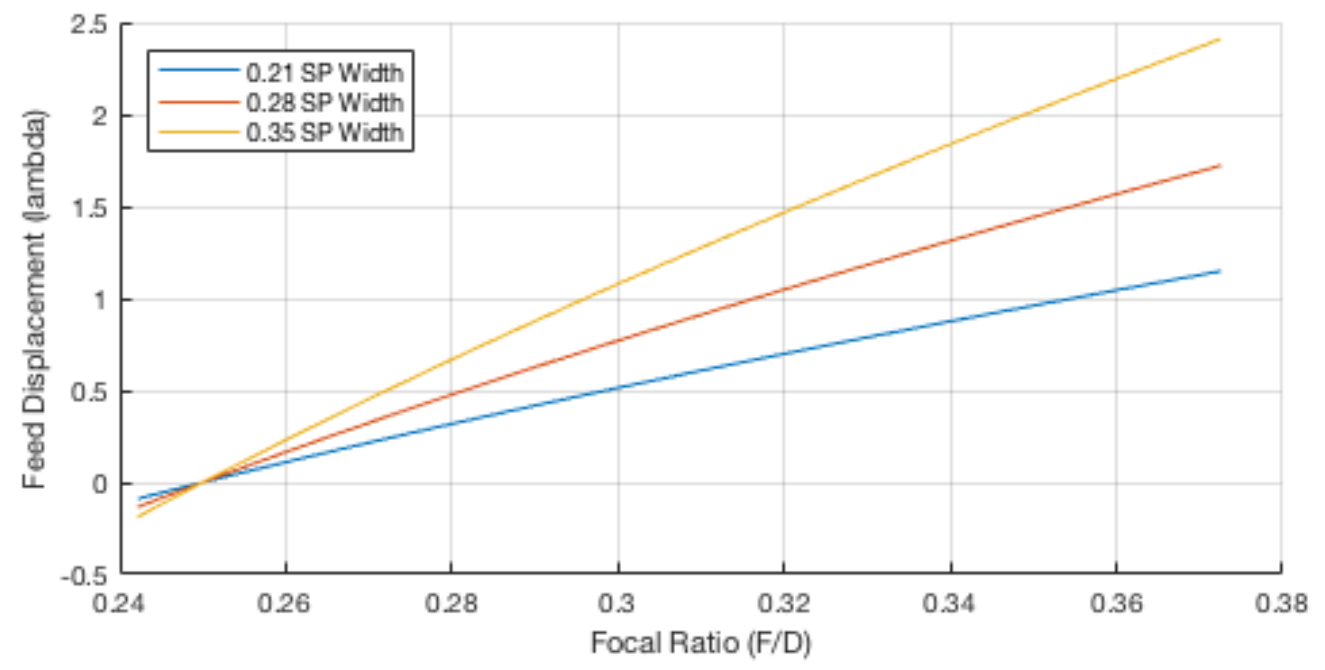

\section{Figure 6.9: Axial Displacement in X-Band Wavelengths as a Function of Solar Panel Widths}

Observing the trends in Figure 6.9, it is apparent that the smaller solar panel widths result in smaller focal point displacements as the F/D ratio increases. Comparing these results with the curves shown in Figure 6.7, it is apparent that a low focal ratio must be selected to maximize efficiency. While it is important to maximize the parabolic dish efficiency, it is important to keep in mind that the spacecraft must generate sufficient electrical power. For this reason, it is beneficial to select the solar panel width case that has enough illuminated solar cell area yet does not require the focal ratio to be too low, thus sacrificing dish efficiency. Observing the results in Figure 6.9 and the curve in Figure 6.7, the solar panel width case of $20 \mathrm{~cm}(28 \mathrm{~cm}$ diagonal) at a focal ratio of 0.27 was chosen. This results in a feed displacement of about $0.32 \mathrm{X}$-Band wavelengths from the focal point. Tracing this back to the curve in Figure 6.7, the parabolic dish efficiency is roughly 50\%. However, as is stated in the graph, real world efficiencies are at least $15 \%$ lower. Therefore, the parabolic dish efficiency will be assumed as 35\%. The solar panel width case of $20 \mathrm{~cm}$, or $28 \mathrm{~cm}$ di- 
agonally, maximizes the area of solar cells in the focal area without wasting potential sunlight to the sides of the spacecraft, as seen in the $25 \mathrm{~cm}$ solar panel width case in Figure 6.8. The focal ratio results in a $\theta_{o}$ angle of about 85 degrees. However, with several sets of solar panels at various angles, the solar panels should be able to generate electrical power without significant incidence angle losses. The effective solar cell area efficiency can also be calculated by taking the ratio of the solar cell area and the total focal area. Using a diameter of $28 \mathrm{~cm}$ and the area of the patch antenna, the effective solar cell area is about $84 \%$ of the illuminated area of the face.

A summary of the parabolic dish shape and efficiency parameters can be seen in Table 6.3. A visual of this shape can be seen in Figure 6.10.

Table 6.3: Summary of Deployable Parabolic Reflector Parameters

\begin{tabular}{|c|c|}
\hline F/D Ratio & 0.27 \\
\hline$\theta_{o}$ & 85 degrees \\
\hline Parabolic Dish Efficiency & $35 \%$ \\
\hline Effective Solar Cell Area Efficiency & $84 \%$ \\
\hline
\end{tabular}




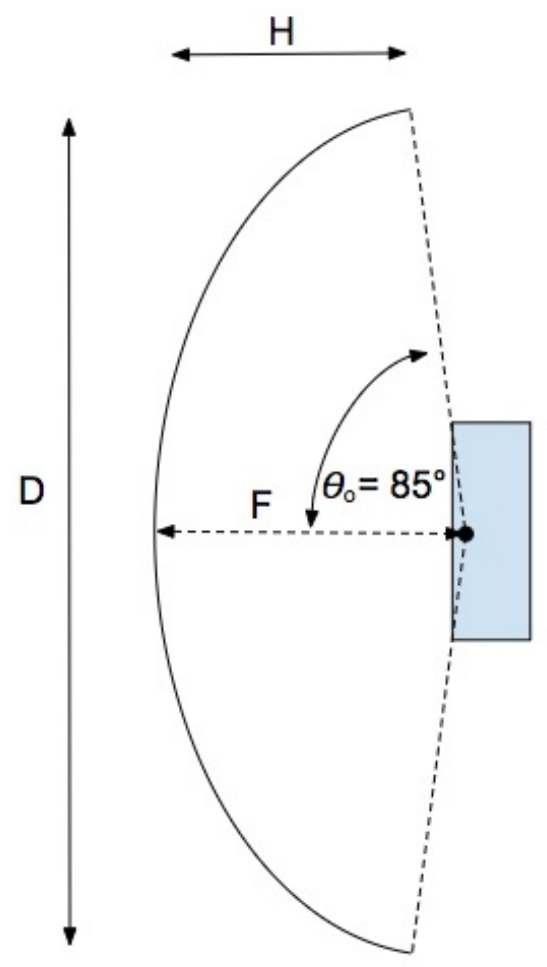

\section{Figure 6.10: Resulting Parabolic Dish Shape}

This focal ratio results in a rather deep parabola and high angle from the edge of the dish to the focal point, $\theta_{o}$. The resulting diameter of the dish will be sized in the power subsystem chapter according to the power requirements.

\subsubsection{Spherical Antenna Alternative}

While the parabolic dish shape is ideal for this type of spacecraft, a spherically shaped antenna may be more feasible to produce for the inflatable antenna concept, as Alessandra Babuscia's most recent paper has described. Although a spherical dish may not have a precise focal point, the optical analysis shows that the rays still converge to a precise area. In fact, spherical dishes become better approximation of parabolic dishes as the depth of the spherical dish decreases. However, as mentioned in the previous section, more shallow parabolic dishes would require a larger feed 
displacement from the focal point, reducing the dish efficiency. Nevertheless, while more analysis is needed for the spherical antenna option, the spherical antenna may be able to concentrate sunlight to a precise enough point to generate electrical power.

Figures produced by Professor Howard Haber from the Santa Cruz Institute of Particle Physics for a physics course shows the behavior of optics in a spherical antenna[10]. Figure 6.11 shows the concentration of rays for a sphere of unitless radius 7 for the nominal case and the 5 degree off axis case. Haber also shows similar cases for the parabolic dish shape in Figure 6.12.
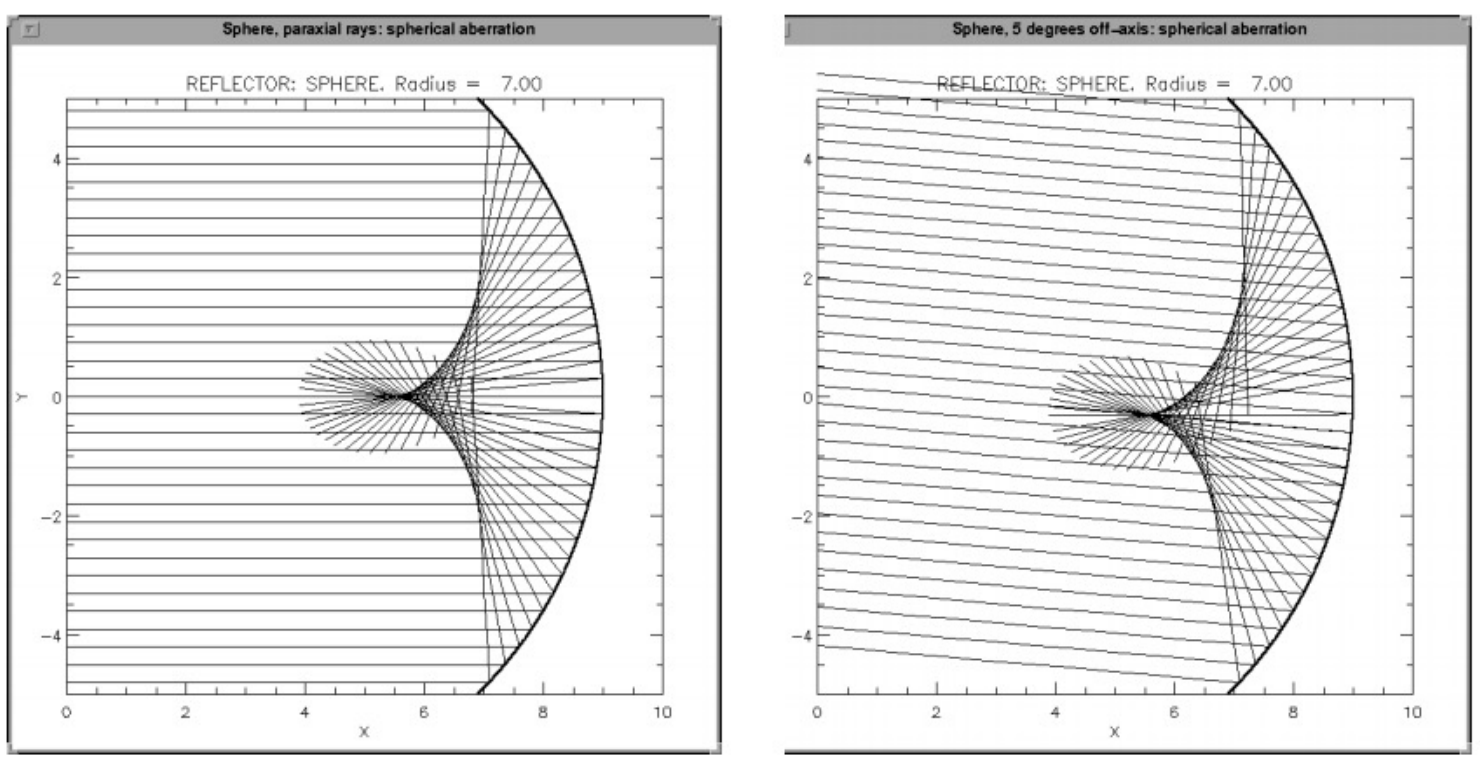

Figure 6.11: Spherical Dish Ray Tracing for Nominal and 5 Degree Off Axis Case by Dr. Howard Haber[10] 

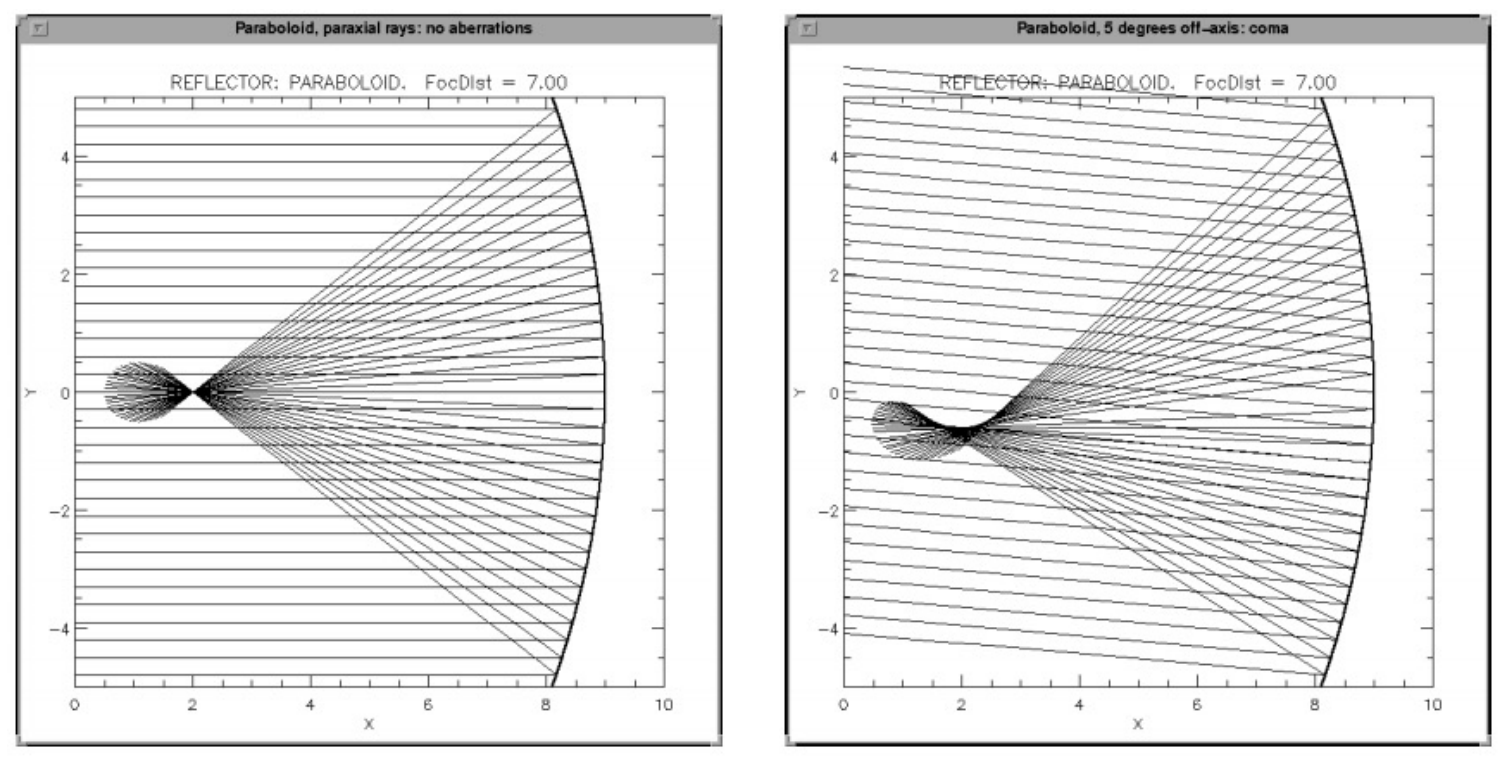

Figure 6.12: Parabolic Dish Ray Tracing for Nominal and 5 Degree Off Axis Case by Dr. Howard Haber[10]

Comparing these figures, it is interesting to note the difference in behavior in the 5 degree off axis case for the different dish shapes. Observing the approximate location of the focal point, the focal point of the spherical dish appear to shift along the Y-axis far less than that of the parabolic dish. This may aid the spacecraft in generating electrical power when close to the Sun, when the Sun-Probe-Earth angle is largest. It should also be noted that the pseudo-focal point of the spherical antenna may be ideal for the spacecraft's need to illuminate the solar cells while maintaining its focal point at an optimal position for the feed. The rays seen in the nominal case of the spherical dish appear to spread out over an area of which the solar cells would reside.

While the off axis case shows promise, one should keep in mind that spherical antennas lack the optimal shape for RF transmissions, thus suffering a loss in dish efficiency. Although the spherical dish would differ in shape from the parabolic dish, feed solutions can be employed to compensate for the inaccuracies of the spherical dish shape. This concept is detailed in Babuscia's latest paper[51].

Although the parabolic dish shape is optimal for the high gain antenna and solar 
concentrator function of the deployable reflector dish, the more practical spherical dish should be investigated using advanced ray tracing and optics software to understand the implications of using this shape. 


\section{Chapter 7}

\section{POWER AND THERMAL SUBSYSTEMS}

Power and thermal requirements are important concerns for a deep space missions. As the spacecraft travels further from the Sun, the amount of available electrical power and thermal energy from sunlight decreases. This typically calls for the use of RTGs to generate power and radioisotope heater units (RHUs) to generate thermal energy. However, a key goal for this spacecraft is to explore a design that does not use any nuclear power or heating units, due to their high costs and hazardous qualities as secondary payloads. Without RTGs, the spacecraft will need to use its deployable reflector dish to concentrate sunlight in order to generate power for this mission. In addition, the spacecraft will need to rely on thermal dissipation from components and heaters to maintain temperatures within operational limits.

\subsection{Power Subsystem}

Due to the low amount of power available at $100 \mathrm{AU}$, this spacecraft will need to be a low-power system that operates on standby mode for a majority of the mission. The spacecraft will then periodically take measurements for short periods of time. Transmissions back to Earth will occur as infrequently as once per week, returning a data volume of $1 \mathrm{~KB}$.

\subsubsection{Power Consumption Requirements}

The power subsystem is designed to satisfy several power requirements. These power requirements consist of supplying enough electrical power to sustain several power modes, operating the payload and transmitter for a specific operations cadence, and 
operating nominally at a certain distance. These power requirements will then flow down to solar concentrator aperture sizing requirements as well as sizing batteries and solar panel area. The power consumption breakdown by component for the various power modes at $100 \mathrm{AU}$ are listed in Table 7.1. Many of the components are based off of power consumptions from COTS components currently available for CubeSats today. While the transmitter will need to be a highly specialized component for the high power transmitting mode, the standby power is also based off of current CubeSat components.

Table 7.1: Power Breakdown for Spacecraft Power Modes at 100 AU

\begin{tabular}{|c|c|c|c|c|}
\hline & \multicolumn{4}{|c|}{ Power Modes } \\
\hline Component & Transmit (W) & Measurement (W) & Standby (W) & Burn (W) \\
\hline \multicolumn{5}{|c|}{ Payload } \\
\hline Magnetometer & - & 2 & - & - \\
\hline Plasma Instrument & - & 2 & - & - \\
\hline \multicolumn{5}{|c|}{ ADCS } \\
\hline Sun Sensor & 0.13 & 0.13 & 0.0375 & 0.13 \\
\hline Thruster System & - & - & - & 4.5 \\
\hline \multicolumn{5}{|c|}{ Comms } \\
\hline Transmitter/Receiver & 76 & 0.2 & 0.2 & 0.2 \\
\hline \multicolumn{5}{|c|}{ C\&DH and EPS } \\
\hline Processor & 0.43 & 0.43 & 0.13 & 0.43 \\
\hline PDU & 0.015 & 0.015 & 0.015 & 0.015 \\
\hline Total Power & 76.775 & 4.775 & 0.3825 & 5.275 \\
\hline Duty Cycle & 800 secs/Op Cycle & 4 mins/hour & Rest of Op Cycle & Infrequent \\
\hline
\end{tabular}

Table 7.1 details the power consumption breakdown for the various power modes and the associated duty cycle that must be supported during the mission. The length of the operation cycle is defined by how often data is transmitted back to Earth, and this requirement is set as anywhere between once a day to once a week as stated in 
the system requirements chapter. The length of the operation cycle is also dependent on how much electrical power can be generated while on standby mode. The transmit mode is dependent on the size of the parabolic dish, as the larger the aperture is, the lower the RF power required to close the communications link. For an $8 \mathrm{~m}$ dish, the RF power required is roughly $23 \mathrm{~W}$, and assuming a $30 \%$ efficient transmitter, this results in a $76 \mathrm{~W}$ power consumption. The $30 \%$ transmitter efficiency is consistent with high-performance solid state power amplifiers[53]. The measurement mode consists of both instruments operating at $2 \mathrm{~W}$ of power each for 4 minutes every hour, 2 minutes for warm-up and preparation and another 2 minutes for taking measurements. Standby mode consists of operating the necessary bus components at their standby power consumption levels. Burns will occur infrequently, consisting mainly of warm-up time of the catalyst bed and then a short impulsive maneuver to adjust the spin stabilized attitude of the spacecraft. Burns are dependent on the orbital trajectory, however, they occur infrequently when at 100 AU. This again is dependent on the beamwidth of the antenna and thus the size of the dish. This will be covered in more detail in the ADCS chapter.

In order to determine the amount of electrical power that must be generated, the average power for different length of operations cycles must be analyzed. Assuming a $76 \mathrm{~W}$ power consumption during transmissions, Table 7.2 details the average power for the various operations cycle frequencies. The average power required assumes that the electrical power passes through a $90 \%$ efficient power distribution unit (PDU) twice, once when charging the battery and another instance when discharging the battery. However, during standby mode, the power is assumed to pass directly to the components, bypassing the PDU. The power required during the burn mode can be neglected due to its infrequent operations.

Table 7.2 shows that as the operations cycle frequency decreases, the average power across all systems for the spacecraft decreases. This is due to increasing the 
Table 7.2: Average Power Consumption for Different Operations Cycle Lengths

\begin{tabular}{|c|c|c|c|}
\hline Operations Cycle Length & 1 Day & 3 Day & 1 Week \\
\hline Total Measurement Mode Energy (Whr) & 9.43 & 28.30 & 66.02 \\
Total Transmit Mode Energy (Whr) & 21.33 & 21.33 & 21.33 \\
Total Standby Mode Energy (Whr) & 8.48 & 25.62 & 59.89 \\
\hline Total Energy per Day (Whr/day) & 38.70 & 24.90 & 20.96 \\
\hline Average Power Consumption (W) & 1.64 & 1.04 & 0.87 \\
\hline
\end{tabular}

time between the high power transmissions. As apparent in the table, the average power consumption for a 1 week operations cycle is less than a Watt.

\subsubsection{High Efficiency Radiation Tolerant Solar Cells}

This spacecraft will employ the newest generation of high efficiency solar cells to maximize power generation per unit area. Recent developments in Low-Intensity Low-Temperature (LILT) optimized solar cells offer significant efficiency improvements compared to traditional high-end solar cells. Andreea Boca and a team of 6 others from NASA JPL and SolAero Technologies Corp. have been developing solar cells optimized to perform at distances of 5-10 AU under low sunlight intensity and low temperature conditions[54]. Boca et al. have set goals of developing solar cell technology that operates at over ¡36\% efficiency under Jupiter and Saturn conditions, $20 \%$ higher efficiency than state of the art systems. Not only do they have improved performance in power generation per unit area, but they also have superior radiation tolerance. The team ran beginning-of-life (BOL) and end-of-life (EOL) efficiency tests for the Jupiter case of 5.5 AU sunlight (0.033 suns) and an operating temperature of $-140{ }^{\circ} \mathrm{C}$. Solar cells underwent radiation dosages of $1 \mathrm{E} 151 \mathrm{MeV} \mathrm{e}^{-} / \mathrm{cm}^{2}$ to represent EOL radiation degradation. These initial tests yielded average efficiencies 
of $35 \%$ at BOL and $31-33 \%$ at EOL. Assuming the radiation dose represents a 10 year mission, this translates to a degradation rate of just under $1 \%$ per year. The mission lifetime assumption may be inaccurate, however, these solar cells are intended for missions near, and possibly orbiting, Jupiter and Saturn. This means degradation rates may in fact be lower for a mission travelling mostly through interplanetary space. Nevertheless, the estimated degradation rate can be cross-referenced using a different source from Scott Benson at NASA Glenn Research Center, Solar Power for Outer Planets Study, a presentation to the Outer Planets Assessment Group[55]. In the presentation, Benson lays out a quick study of a Saturn orbiter mission using photo-voltaic (PV) and lists assumptions of an 11.5 year voyage to Saturn with a total radiation degradation of $15 \%$. It is unclear whether this degradation is simply during the voyage or includes the radiation from orbiting Saturn. For a conservative estimate, assuming the degradation total is as a result of the 11.5 year voyage translates to a $1.4 \%$ degradation per year. It should be noted that this study was performed in 2007 and assumed near-term PV technology.

Taking into account the initial test results from Boca et al. in 2017 and the assumed degradation loss from Benson's presentation in 2007, it is reasonable to assume that the small OSS probe can utilize $36 \%$ efficient solar cells with a degradation rate of $1.2 \%$ per year. These assumptions will be used to determine EOL power estimates in the following sections.

\subsubsection{Power Generation Requirements}

Now that power consumption values for various operations cycles have been generated, the parabolic solar concentrator can now be sized. Taking into consideration several inefficiencies, the amount of electrical power generated can be estimated. First, the efficiencies must be explained to understand the true available electrical power. These 
efficiencies are listed in Table 7.3 Because it takes around 30 years using a Voyager 1-like trajectory to reach $100 \mathrm{AU}$, the EOL solar cell efficiency will assume 30 years of solar cell degradation.

\begin{tabular}{|c|c|c|c|}
\hline & Efficiency Type & Variable & Value \\
\hline & Solar Concentrator Eff. & $\eta_{c}$ & 0.800 \\
\hline & Packing Factor Eff. & $\eta_{P F}$ & 0.925 \\
\hline & Incidence Angle Eff. & $\eta_{\theta}$ & 0.925 \\
\hline & EOL Solar Cell Eff. & $\eta_{s c}$ & 0.251 \\
\hline & Effective Area Eff. & $\eta_{A}$ & 0.840 \\
\hline
\end{tabular}

Most of the effiencies listed in Table 7.3 are based off of typical efficiencies seen in industry, while other mission specific efficiencies are approximated through calculations. $\eta_{c}$ represents the solar concentrator efficiency. Aluminized mylar, a prime candidate for the reflector dish and the material used in Babuscia's inflatable CubeSat antenna, is roughly $95 \%$ reflective over the visible spectrum[34]. The concentrator efficiency also takes into account surface irregularities in which the sunlight does not reflect as intended onto the solar cell surface. However, the $80 \%$ of light reflected specularly off the dish as intended is assumed to reach the solar cell surface. $\eta_{P} F$ is the packing factor efficiency and is often around $90 \%$ to $95 \%$ [53]. The incidence angle efficiency takes into account sub-optimal sunlight incidence angles onto the solar cells. Because the parabolic dish shape is quite deep, not all sunlight will hit the solar cells at 90 degrees incidence angle. The incidence angle efficiency, $\eta_{\theta}$ of 0.925 corresponding to an average incidence angle of 22.3 degrees. With a worst maximum angle between the edge of the dish and the focal point $\left(\theta_{o}\right)$ of 85 degrees, two sets of solar panels, if correctly angled at 100 AU can take up 42.5 degrees of parabolic dish. This corresponds to a worst case incidence angle of just over 21 degrees on either 
end of the angle normal to the solar cell face. It should be noted that the incidence angle is assumed to be an average of 22.3 degrees, taking into account inefficiencies due to the non circular configuration of solar cells around the feed. Nonetheless, the true incidence angle efficiency should be determined using advanced ray tracing software. The EOL solar cell efficiency, $\eta_{s c}$, is based on the 30-year travel time to 100 AU using a Voyager 1-style mission using Jupiter and Saturn for gravitational assist maneuvers. This efficiency is a function of BOL efficiency and mission lifetime and can be calculated using Equation 7.1. D represents the degradation per year, and L represents the spacecraft lifetime in years[53].

$$
\eta_{E O L}=\eta_{B O L}(1-D)^{L}
$$

Baselining the travel time Voyager 1 took to reach $100 \mathrm{AU}$, the spacecraft lifetime will be approximated to be 30 years. This trajectory will be treated as worst case, as other trajectory options, such as those mentioned in the interstellar probe studies by NASA and KISS baseline 10 years travel time to $100 \mathrm{AU}$, reducing the EOL solar cell efficiency. However, as stated in the previous section, the spacecraft will use LILT optimized solar cells with $36 \%$ efficiencies with advanced radiation tolerance, yielding a $1.2 \%$ performance degradation per year. Using these parameters, the EOL solar cell efficiency can be approximated to $25.1 \%$.

The final efficiency that must be considered is the effective area of the solar panel surface seeing the sun, $\eta_{A}$. Because the patch antenna feed takes up $100 \mathrm{~cm}^{2}$ of area on the reflector facing surface, there is an effective area that actually consists of solar cells. Assuming the parabolic dish concentrates sunlight onto a circular region of the solar panel face, it must be understood that $100 \mathrm{~cm}^{2}$ of the sunlit region will consist of the patch antenna feed. Due to the shape of the dish and the position of the focal point, the concentrated sunlight can be assumed to take up a $28 \mathrm{~cm}$ diameter circle 
on the solar panel and feed face. While the sunlight illuminates an area of $615.7 \mathrm{~cm}^{2}$, $100 \mathrm{~cm}^{2}$ of area will be occupied up by the feed antenna, leaving an effective solar cell area efficiency of $84 \%$. This configuration can be seen in Figure 7.1.

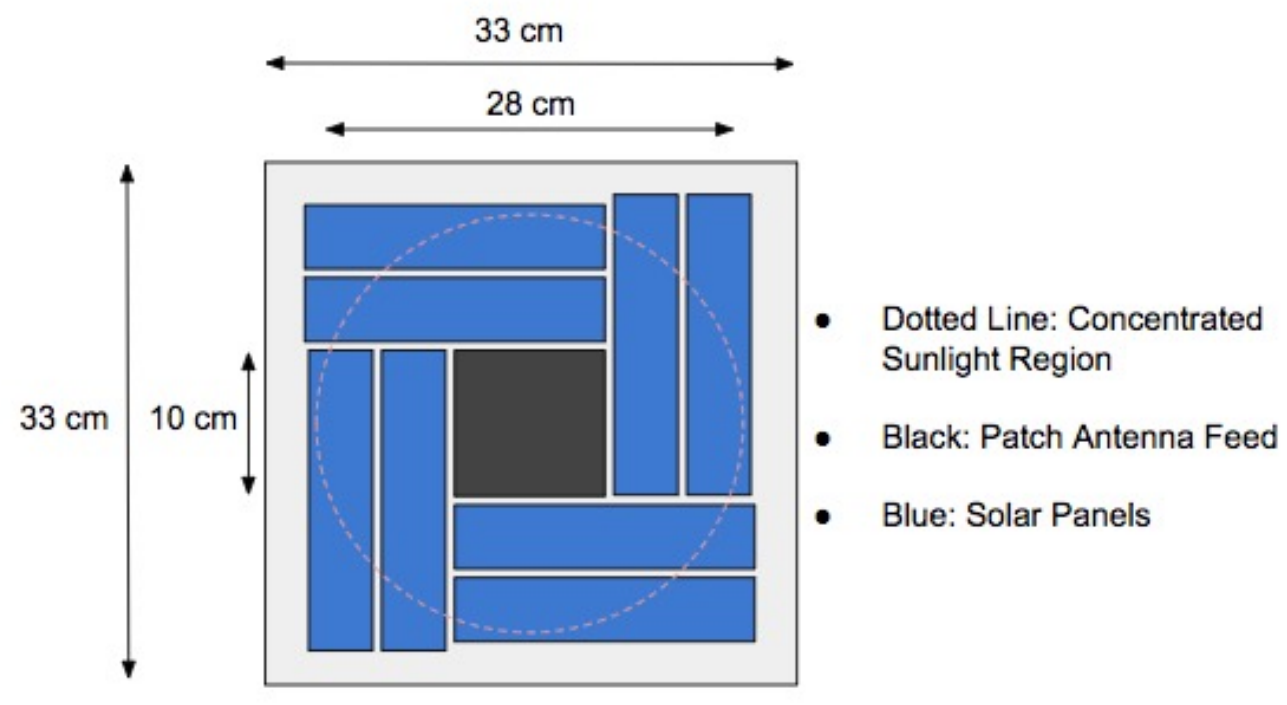

Figure 7.1: Effective Sunlit Area on Solar Panel and Feed Face

After defining all the efficiencies and determining appropriate values, the power generation can be approximated as a function of solar intensity, reflector dish size, and the various efficiencies using Equation 7.2.

$$
P_{\text {gen }}=A_{d} I_{s} \eta_{c} \eta_{P F} \eta_{\theta} \eta_{s c} \eta_{A}
$$

Examining the average power generation at $100 \mathrm{AU}$ with various dish sizes yields Table 7.4.

Comparing the values shown in Table 7.4 with the values listed in Table 7.2 shows that operation cycles of 1 week coupled with a $7.75 \mathrm{~m}$ diameter concentrator dish appear to be the most feasible option of satisfying average power consumption 
Table 7.4: Average Power Generated as a Function of Concentrator Dish Size

\begin{tabular}{|c|c|c|c|c|}
\hline Concentrator Dish Diameter (m) & 7 & 7.25 & 7.5 & 7.75 \\
\hline Concentrator Dish Area $\left(\mathrm{m}^{2}\right)$ & 38.48 & 41.28 & 44.18 & 47.17 \\
Energy Generated per Day (Whr) & 18.19 & 19.52 & 20.89 & 22.30 \\
Power Generated (W) & 0.758 & 0.813 & 0.870 & 0.929 \\
\hline
\end{tabular}

requirements while adding a little margin.

\subsubsection{Battery Sizing}

While most of the operational modes are relatively low power, the battery must be sized for the highest power operation, the RF transmission back to Earth. To the immense distance of $100 \mathrm{AU}$ back to Earth, the transmitter must generate $23 \mathrm{~W}$ of RF power in order to satisfy the link with enough margin. Assuming a state of the art $30 \% \mathrm{SSPA}$, this results in about $76 \mathrm{~W}$ of electrical power. Adding the power consumption from other components such as the sun sensor, processor, and PDU, this results in a total electrical power draw of $76.76 \mathrm{~W}$. If the PDU is assumed to be $90 \%$ efficient, the true power draw becomes $85.3 \mathrm{~W}$. The transmission mode lasts 800 seconds to transmit all the data, so the battery must be capable of delivering 17.06 Whrs of energy at 100 AU. Assuming that the spacecraft takes a measurement just before the transmission, the total energy that the battery must deliver can be increased by 0.32 Whrs to a total of 17.38 Whrs. The maximum power draw and total energy requirements can be then used to size the battery.

However, the spacecraft will undergo many cycles by the time it reaches $100 \mathrm{AU}$. This is important because battery Depth-of-Discharge (DoD) decreases over the cycle life. Because the measurement and burn modes have low power consumptions during short periods of time, it can be assumed that these modes are not considered as a 
cycle and will have minimal effect on the cycle life of the battery. Therefore, if we only take into account the cycles as a result of the high power transmission mode, which occur once ever 7 days, the total cycles that the battery experiences over 30 years will be about 1565 cycles. For lithium ion batteries, this results in a minimal DoD degradation. However, due to the length of time the battery must operate and ageing of the battery, we will assume the $\mathrm{DoD}$ degrades to $50 \%$. In addition, the PDU will have a maximum efficiency of $90 \%$. With these parameters, the battery capacity requirement in watt-hours can be calculated with Equation 7.3[53].

$$
C=\frac{E_{T}+E_{M}}{(D O D) N n}
$$

$E_{T}$ and $E_{M}$ represent the energy in Whrs of the transmission mode and measurement mode, respectively. $D O D$ represents the worst case Depth-of-Discharge of the battery at EOL. $N$ represents the number of batteries, and $n$ is the PDU efficiency. Assuming the spacecraft uses only one battery, the total battery capacity will need to be 38.22 Whrs. If the bus is assumed to operate at $14.8 \mathrm{~V}$, the battery must have a capacity of 2.6 Ah. With the battery capacity sized, observing current COTS power components can help give an idea of what maximum power as well as mass and volume sizes can come from these types of batteries.

Gomspace, a Denmark-based company, sells many nanosatellite components for a variety of subsystems. They advertise two lithium ion batteries, the BP4 and BPX, both with a maximum discharge current of $8 \mathrm{~A}[56][57]$. The $\mathrm{BP} 4$ is a $38.5 \mathrm{Whrs}$ battery consisting of four individual batteries that can be configured as four batteries in series or two sets of two batteries configured in parallel. Their BPX battery is slightly larger battery at $77 \mathrm{Whrs}$, consisting of eight batteries that can be configured in either 8 series 1 parallel, 4 series 2 parallel, or 2 series 4 parallel. While the BP4 battery appears perfectly sized for the 38.22 Whr capacity requirement, the battery 
should be sized to allow for enough margin to account for estimation inaccuracies, such as the battery aging effects over the 30 year lifetime. For these reasons, the BPX battery will provide substantial margin for little added mass and volume by providing 77 Whrs of energy. In order to fulfill the maximum power draw requirement of 85.3 $\mathrm{W}$ with necessary margin, the battery will need be relatively high voltage. If the solar panels can be configured to $14.8 \mathrm{~V}$, the Gomspace BPX battery can be configured as a four series 2 parallel battery to provide 5.2 Ah of current capacity. With a discharge current of $8 \mathrm{~A}$, this battery can provide a maximum of $118.4 \mathrm{~W}$ electrical

power, satisfying the $85.3 \mathrm{~W}$ maximum power requirement for the transmissions[58]. This battery has a mass of $0.5 \mathrm{~kg}$ and a volume of roughly $0.5 \mathrm{U}$. For these reasons, the Gomspace BPX battery will be baselined for this spacecraft to assume the mass and volume of a battery required for this spacecraft.

\subsubsection{Power Subsystem Summary}

The important parameters for the required power subsystem is listed in Table 7.5. These values provide a general overview of what the power subsystem looks like for this spacecraft.

With the dish diameter sized, we can approximate the packaged volume and mass of the reflector antenna system. As detailed by Babuscia and shown in Table 6.2, the current system can scale up to $8 \mathrm{~m}$ diameter, resulting in a $20.5 \mathrm{U}$ and $20.5 \mathrm{~kg}$ system[3]. Assuming packing efficiencies and thin membrane technologies will improve in the near future, we will assume the system requires $13 \mathrm{U}$ and with a corresponding mass of $13 \mathrm{~kg}$, a $35 \%$ improvement to the packing efficiency and mass of the system. 


\section{Table 7.5: Major Power Subsystem Characteristics}

\begin{tabular}{|c|c|}
\hline \multicolumn{2}{|c|}{ Requirements } \\
\hline Average Power Consumption & $0.87 \mathrm{~W}$ \\
Maximum Power Draw & $76.75 \mathrm{~W}$ \\
Energy Storage Requirement & $38.22 \mathrm{Whr}$ \\
\hline \multicolumn{2}{|c|}{ Solutions } \\
\hline Concentrator Dish Diameter & $7.75 \mathrm{~m}$ \\
Concentrator Focal Length & $2.09 \mathrm{~m}$ \\
Concentrator Focal Depth & $1.79 \mathrm{~m}$ \\
Average Power Generation & $0.929 \mathrm{~W}$ \\
Battery Baseline & Gomspace BPX \\
Battery Maximum Power & $118.4 \mathrm{~W}$ \\
Battery Energy Capacity & $77 \mathrm{Whr}$ \\
Battery Mass & $0.5 \mathrm{~kg}$ \\
Battery Volume & $0.5 \mathrm{U}$ \\
\hline
\end{tabular}

\subsection{Thermal Subsystem}

Now that the power subsystem has been determined, the thermal subsystem must be able to deal with the large amounts of heat from the high power modes while also keeping the spacecraft warm during its ultra-low power standby modes in deep space. Because the secondary payload option is ideal for this type of spacecraft, it will need to operate in a variety of thermal environments to maximize rideshare opportunities.

There are a number of considerations when starting to design the thermal aspect of this spacecraft. First, the necessary bus electronics can be housed in a smaller and separate compartment than that which houses the inflatable antenna. This will minimize the amount of heat lost through radiation to space and allow the low thermal 
dissipation from electronics during standby to keep the components warm. This smaller bus will need to be highly thermally insulated with high performance multilayer insulation (MLI) to keep the bus warm. This means that the excess heat at the solar panel face cannot be transferred to the electronics bus as the compartment will reach too high of temperatures. Second, the electronics bus will need to be conductively thermally isolated from the solar cell surface. Solar cells very emissive, at roughly 0.8 emissivity, and would act as a radiator for the spacecraft bus if the two components were conductively coupled[4]. This can be accomplished using thermally insulative materials such as carbon fiber to attach the bus to the solar cell face. At $100 \mathrm{AU}$, there will be little available electrical and thermal power, so isolating the solar cell face from the bus will allow the solar cells to operate at low temperatures as well as keep crucial thermal energy from escaping the spacecraft bus.

A major problem when close to the Sun is that solar cells are highly absorptive at around 0.8 and will absorb large amounts of the heat from sunlight that not converted to electrical energy. A simple thermal equilibrium calculation shows that the solar panel and feed face of the spacecraft reaches extremely hot temperatures. Using Equation 7.4 and Equation 7.5.

$$
\begin{gathered}
Q_{\text {in }}=I_{s} A_{\text {solarcells }}\left(\alpha-\eta_{\text {cells }}\right) \\
Q_{\text {out }}=\sigma A_{\text {face }} \epsilon T^{4}
\end{gathered}
$$

Assuming all of $80 \%$ the focused energy hitting the parabolic dish reaches the solar cell plate and is focused mostly on solar cells, we can assume that the absorptivity, $\alpha$, is 0.8 for the entire surface seeing sunlight. For this analysis, the maximum solar cell efficiency will be assumed. The spacecraft is also assumed to be at Jupiter, with the parabolic dish receiving about $50 \mathrm{~W} / \mathrm{m}^{2}$. Because the bus must be as thermally 
isolated as possible from the solar cell face, conduction to the bus and radiation out the back of the plate will be neglected. The emissivity, $\epsilon$, will be set to solar cell emissivity values of 0.8 and the area of the face is assumed to be $33 \mathrm{~cm} \mathrm{x} 33 \mathrm{~cm}$. Using these parameters the temperature of the solar cell and feed face reaches $831 \mathrm{~K}$. This obviously calls for extra radiating surfaces.

However, because the spacecraft is designed to operate at $100 \mathrm{AU}$, the spacecraft will be receiving a surplus of thermal energy if showing the entirety of solar cell area. This means the spacecraft can minimize the solar cell area facing the sunlight to decrease the amount of thermal energy absorbed while still receiving enough electrical power to support the low frequency operations of the spacecraft. This issue can be solved by placing these solar cells on the outer face of louvers. These louvers will be capable revolving 180 degrees, and would show a white paint radiator surface on the back of the solar panels as well as a white radiator surface on the underside of the solar cells. This white paint would reduce the thermal energy absorbed from the sunlight and would also act as a highly emissive radiative surface to radiate the heat out of the spacecraft. The louvers can be actuated using bimetallic springs that react to temperatures.

\subsection{Thermal Requirements}

First, the thermal requirements of the spacecraft must be examined to determine what temperatures the components must be kept at. Table 7.6 shows a list of the spacecraft components, their thermal power, and operating temperatures.

Most operating limits are referenced from currently available COTS nano-satellite parts, with the exception of the solar cells and the batteries. The spacecraft will be using the newest generation of LILT solar cells, optimized for operating at low temperatures and low solar intensities. The solar cells currently being developed by 
Table 7.6: Component Level Thermal Requirements

\begin{tabular}{|c|c|c|c|}
\hline Component & Ave. Thermal Power (W) & Peak Thermal Power (W) & Temp. Limits (C) \\
\hline Sun Sensor[28] & 0.0375 & 0.13 & -25 to 70 \\
Processor[27] & 0.13 & 0.43 & -25 to 60 \\
PDU[59] & 0.015 & 0.015 & -40 to 85 \\
Transmitter[29] & 0.2 & 53 & -20 to 60 \\
Battery[60] & - & - & -30 to 30 \\
Thruster System[61] & - & 4.5 & -30 to 60 \\
Solar Cells[54] & - & - & -200 to 100 \\
Magnetometer[62] & - & 2 & -35 to 75 \\
Plasma Instrument & - & 2 & -35 to 75 \\
\hline
\end{tabular}

Boca et al. operate optimally at $-165 \mathrm{C}$, meaning they can likely operate at even lower temperatures[54]. The upper operating limit for the solar cell are derived from typical maximum solar cell operating temperatures. The propellant tanks will house AF-M315E green monopropellant that is known to be able to reach low temperatures of up to -80 C, ideal for a low-power spacecraft during long cruise phases[63]. However, for conservative measures, the assumed operating temperatures will reflect those of the VACCO Argo Moon propulsion system, which uses this type of propellant. The operating temperatures for the lithium ion batteries are also unusual. Typically, lithium ion batteries tend to operate between 10 and $25 \mathrm{C}[53]$. However, the recent INSIGHT mission to Mars will be using new lithium ion battery technology capable of discharging at temperatures as low as $-30 \mathrm{C}$ and up to a maximum of $30 \mathrm{C}[60]$. These batteries are ideal for this type of deep space mission, allowing the batteries to reach low temperatures when little heating power is available. It should be noted that the peak power for the transmitter is only for the hot case, as the closer distances require lower $\mathrm{RF}$ power to close the communications link. 


\subsection{Thermal Desktop Results}

A model of the spacecraft was created in Thermal Desktop for high fidelity thermal analysis. Because the spacecraft experiences a wide variety of thermal environments, the hot and cold case must be investigated to make sure all the components remain within their operating temperatures. A slightly simplified model without the boom deployment mechanism and thruster nozzles was created to perform this analysis. Analysis was done at the hot case, defined as at Jupiter or 5.26 AU, and the cold case, defined as at 100 AU. These cases should represent the extreme temperatures that the spacecraft experiences during the trip from Jupiter to 100 AU.

Due to difficulties in modeling the reflection of the sunlight off of the parabolic reflector dish, a solar intensity was instead calculated from the expected reflected sunlight off of the parabolic dish. In order to factor in the absorptivity of the panels facing the reflector, the spacecraft was placed in a heliocentric orbit in which the solar panel and feed face was constantly directed towards the sun. The solar intensity from the sun in this orbit was then calculated by determining the amount of sunlight concentrated by the dish and dividing this by the area facing the sun. The pseudo solar intensities for the hot case and the cold case were calculated to be $6148 \mathrm{~W} / \mathrm{m}^{2}$ and $16.8 \mathrm{~W} / \mathrm{m}^{2}$, respectively.

After inputting the components and their respective thermal loads into Thermal Desktop, optical properties were tested to determine a viable thermal subsystem design. The resulting surface coatings and optical properties were used on the bus and the radiator panels, shown in Table 7.7 .

As seen in Table 7.7, the bus and payload will be coated in very low emissivity MLI, at an effective emissivity of 0.01. Recent developments in MLI technology have produced extremely low emissivity MLI. A paper presented at the 45th International 


\section{Table 7.7: External Optical Properties for Bus and Radiator Panels[4]}

\begin{tabular}{|c|c|c|c|}
\hline Component & Surface Finish & $\alpha$ & $\epsilon$ \\
\hline Radiator - Top & White Paint & 0.09 & 0.9 \\
Radiator - Bottom & Polished Aluminum & 0.15 & 0.05 \\
Solar Cells - Top & Solar Cells & 0.8 & 0.8 \\
Solar Cells - Bottom & White Paint & 0.09 & 0.9 \\
Bus Outer Surface & MLI & 0.15 & 0.01 \\
Payload Outer Surface & MLI & 0.15 & 0.01 \\
\hline
\end{tabular}

Conference on Environmental Systems by Miyakita et al. describe developments of an MLI blanket with significant insulation performance. In the paper titled Evaluation of Thermal Insulation Performance of a New Multi-Layer Insulation with NonInterlayer-Contact Spacer, the authors show MLI blanket tests that exhibit effective emissivities of 0.0008[64]. While not many current MLI blankets can yield a 0.01 effective emissivity, it is reasonable to assume improvements in this area of technology will reach these emissivity values in the near future.

Conduction between bus components is approximated by setting all optical properties for the internal faces to black paint. The deployable radiator panels and solar cells are assumed to have high conductance with the panel beneath the solar cells and feed. As stated in the beginning of the thermal subsystem section, the bus must be highly conductively isolated from the radiator panels in order to maintain adequate temperatures. While the conductivity for this analysis is set at $0.001 \mathrm{~W} / \mathrm{C}$, the method of achieving this conductivity must be investigated further, coupled with structural analysis of the connection. Conductive isolation is key for the spacecraft to survive thermally, as it experiences a wide variety of thermal environments with little available heater power at the cold case. However, using low conductivity materials and minimizing the area to length ratio of the conduction path, this conductive 
isolation could be feasible. This will allow the solar cell and radiator surface to experience large variations in temperatures while minimally affecting the temperature of the thermally sensitive bus.

\subsubsection{Hot Case Temperature Results}

Using the optical properties listed in Table 7.7, the hot and cold cases were simulated on Thermal Desktop. This subsection details the results of the hot case at Jupiter. As previously stated, the dish is sized for collecting electrical power at $100 \mathrm{AU}$, and

thus, is oversized for closer distances to the sun. This is where the extra radiating surfaces are useful. It should be noted that the transmitter does not produce large amounts of thermal power when at Jupiter, therefore, thermal power is relatively constant. The simulation was a transient run that spanned 1E7 seconds.

Figure 7.2 shows the post processing results in Thermal Desktop for the bus. While the graphics give a good idea of the resulting temperatures, the actual maximum and minimum temperatures over the time span are shown in Table 7.8. 


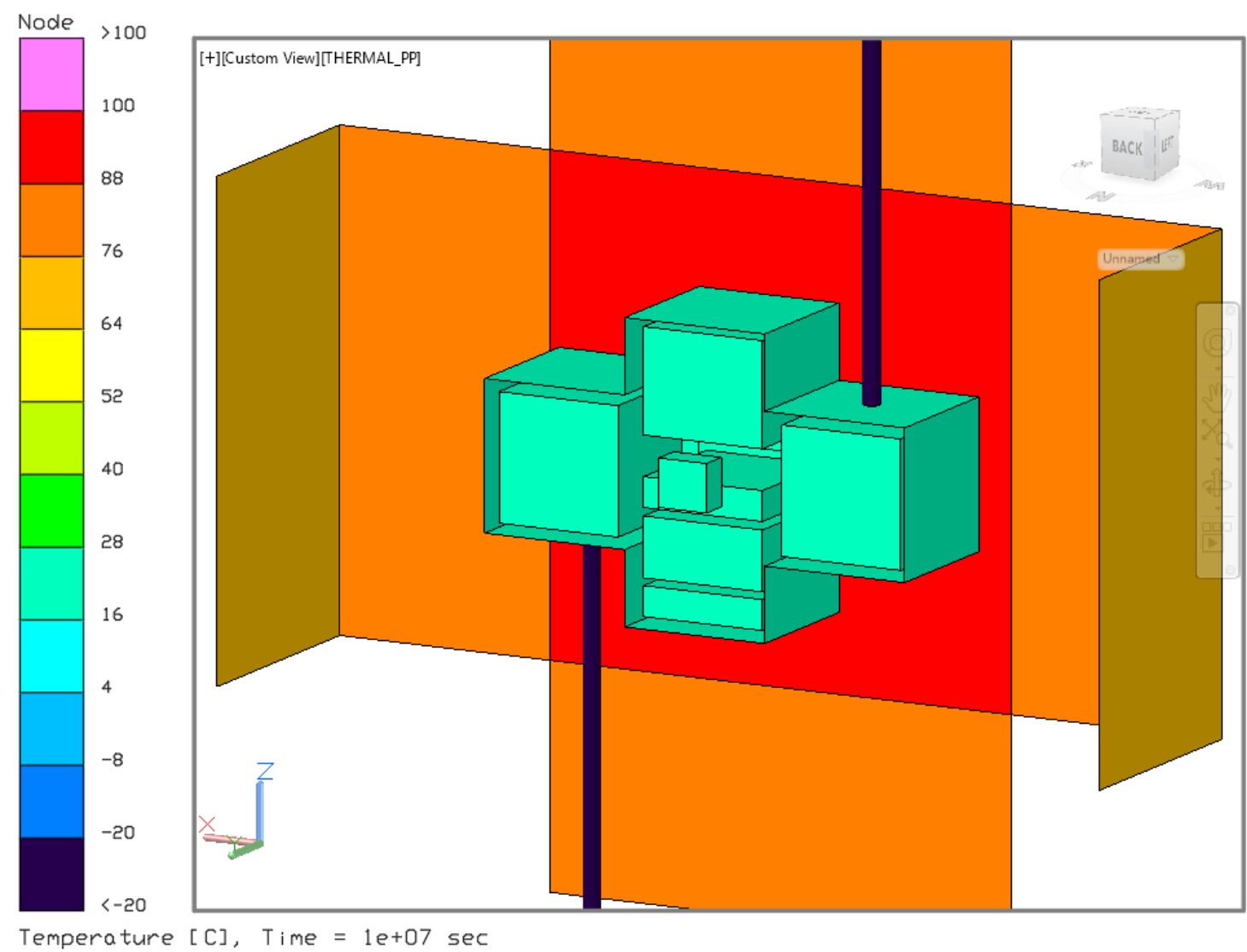

Figure 7.2: Thermal Desktop Results for the Hot Case at Jupiter

The temperature results of the components are generally reasonable, as all components, with the exception of the batteries and solar cells, remain well within operable limits. Although this thermal analysis is performed with limited fidelity, this shows that the spacecraft components can rely on the thermal power generated from component operations while also radiating extra heat at the hot case.

\subsubsection{Cold Case Temperature Results}

Using the same optical properties and conductances as the hot case, a transient case spanning 1E7 seconds was ran for the $100 \mathrm{AU}$ cold case. At this distance, the parabolic reflector dish does not concentrate excess sunlight, instead concentrating 
Table 7.8: Maximum and Minimum Temperatures of Components at the Hot Case

\begin{tabular}{|c|c|c|}
\hline Component & Min. Temp (C) & Max. Temp (C) \\
\hline Sun Sensor & 21.3 & 23.6 \\
Processor & 21.1 & 22.7 \\
PDU & 21.5 & 22.5 \\
Transmitter & 20.4 & 21.3 \\
Battery & 20.7 & 21.5 \\
Propellant Tanks & 18.0 & 18.6 \\
Solar Cells & 91.9 & 91.9 \\
Magnetometer & -3 & 4 \\
Plasma Instrument & -3 & 4 \\
\hline
\end{tabular}

just enough to match the average power consumption of the bus. Another significant difference between the cases is that the transmitter now must operate at its peak power, generating $52 \mathrm{~W}$ of thermal power for 800 seconds, in order to close the communications link due to the large distance between the Earth and the probe. The post processing result from Thermal Desktop for the cold case can be seen in Figure 7.3. Once again, the maximum and minimum temperatures of each component seen in the transient case are shown in Table 7.9. 


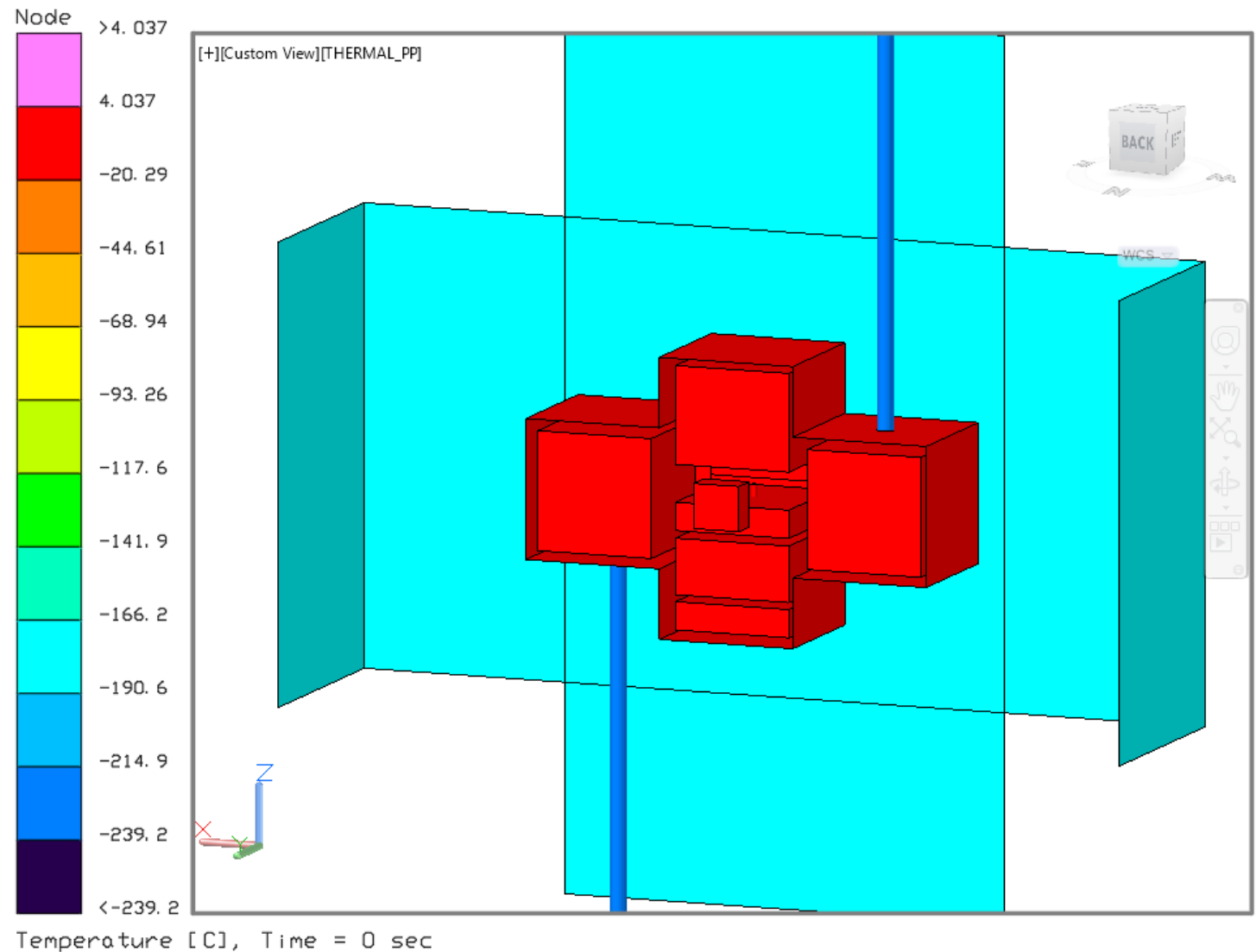

Figure 7.3: Thermal Desktop Results for the Cold Case at 100 AU

Once again, the temperatures are quite reasonable, with decent margin available for most all components. Comparing the cold case payload results with the hot case, it is expected that the temperature would be similar as the instruments are placed far from the focal point of the parabola and the bus and radiator panels. An important result to note is the solar cell temperature of $-179.5 \mathrm{C}$. The JPL LILT solar cells that is use by the spacecraft operates at its highest efficiency at -165 C under Saturn-like conditions[54]. In fact, the trend in the curve shows the efficiency increasing further as the temperature decreases. This means the solar cells will operate at or near its optimal temperature condition. 
Table 7.9: Maximum and Minimum Temperatures of Components at the Cold Case

\begin{tabular}{|c|c|c|}
\hline Component & Min. Temp (C) & Max. Temp (C) \\
\hline Sun Sensor & -10.5 & -2 \\
Processor & -10 & -5 \\
PDU & -10.5 & -4.5 \\
Transmitter & -11.5 & 1 \\
Battery & -11 & -3.5 \\
Propellant Tanks & -16 & -9 \\
Solar Cells & -179.5 & -179.5 \\
Magnetometer & -2.5 & 4 \\
Plasma Instrument & -2.5 & 4 \\
\hline
\end{tabular}

\subsection{Thermal Subsystem Summary}

The thermal subsystem components will be summarized in this section. There are relatively few components that make up the thermal subsystem. However, these few components still add mass to the system. The components and the corresponding dimensions and mass are shown in Table 7.10

Table 7.10: Thermal Subsystem Summary

\begin{tabular}{|c|c|c|c|}
\hline Component & Area & Mass & Comments \\
\hline Solar Cell Louvers & $0.1089 \mathrm{~m}^{2}$ & $0.5445 \mathrm{~kg}$ & Areal Density: $5 \mathrm{~kg} / \mathrm{m}^{2}$ \\
Extra Radiator Panels & $0.3069 \mathrm{~m}^{2}$ & $2.45 \mathrm{~kg}$ & Areal Density: $8 \mathrm{~kg} / \mathrm{m}^{2}$ \\
MLI & $0.22 \mathrm{~m}^{2}$ & $0.36 \mathrm{~kg}$ & Areal Density: $1.5 \mathrm{~kg} / \mathrm{m}^{2}$ \\
\hline
\end{tabular}

The areal densities are assumed by referencing similar systems. The areal density of the louvers can be anywhere from $3-5 \mathrm{~kg} / \mathrm{m}^{2}[53]$. Thermal Management Technologies offers deployable radiator products for spacecraft and lists the areal density of 
their radiators as $8 \mathrm{~kg} / \mathrm{m}^{2}[65]$. The areal density of the MLI was taken from the midpoint of their average areal density graph for their MLI design the Miyakata et al. paper[64]. While these areal densities may be high, it is better estimate conservatively. These masses will be revisited in the mass budget section later on in the paper.

\subsection{Other Thermal Considerations}

The thermal design for this spacecraft limits this spacecraft to operating at $5 \mathrm{AU}$ at its closest. While the spacecraft should ideally be able to operate at $1 \mathrm{AU}$ at the earliest phase of the mission, the fully deployed solar concentrator limits practical solutions for the thermal subsystem at close distances to the sun. However, alternative solutions to this thermal problem should be investigate in the future. For example, the main cause of generating too much thermal energy when close to the sun is due to the deployable antenna essentially pointing directly at the Sun at all times. An alternative solution to this problem would be to point away from the sun and rely on the diffuse reflection of sunlight to power the spacecraft. Although the main lobe of the parabolic dish beam may not be adequately pointed towards Earth, the use of the side lobe to contact Earth at these close distances should be investigated. This may allow the spacecraft to avoid concentrating excess thermal energy and operate closer to the sun, while also retaining the ability to communicate with Earth. Average power consumption during this stage can be reduced further by avoiding the use of the scientific instruments, as the data collected closer to the sun is not valuable to the scientific community. While this is just one possible solution, other possible solutions should be considered to determine how the spacecraft can operate towards the early phases of the mission. 


\section{Chapter 8}

\section{COMMUNICATIONS SUBSYSTEM}

In this chapter, the communications subsystem requirements and ground station options will be determined to estimate the communications subsystem design for the spacecraft. As noted before, the deployable parabolic dish is the most unique aspect of the spacecraft. This dish can be multi-purpose, concentrating low intensity sunlight to generate electrical power, and provide the communications subsystem with a large parabolic dish to act as a high gain antenna. With adequate feed placement and parabolic dish shape, the focal point can accommodate both the solar panels and the feed without incurring significant efficiency losses. The spacecraft will need to operate at distances of up to $100 \mathrm{AU}$ from the sun, requiring high transmitter powers as well as large dish sizes. Fortunately, as outlined in the power subsystem chapter, the reflector dish must be at least $7.75 \mathrm{~m}$ in diameter to generate enough electrical power. Nevertheless, the transmitter will still need to generate large amounts of power to close the link.

\subsection{Link Analysis}

The analysis for this chapter assumes traditional RF link equations, parameters, and efficiencies for a deep space mission. As outlined in the light analysis chapter, several efficiencies and losses are assumed for this deep space communications link. These can be revisited below in Table 8.1. Most of these parameters have been referenced from typical deep space link parameters. Other parameters, such as the receiver efficiency and data rate, are values defined by the Deep Space Network. The $70 \mathrm{~m}$ dishes are just over $70 \%$ efficient at X-Band and even more efficient at S-Band. The lowest data 
rate for downlink is $10 \mathrm{bps}[66]$. The transmit efficiency is calculated in the Deployable Subsystem chapter.

Table 8.1: Communications Parameters for Deep Space Link

\begin{tabular}{cc} 
Parameter & Value \\
\hline $\begin{array}{c}\text { Signal-To-Noise Ratio }\left(\mathrm{SNR} \text { or } \mathrm{E}_{b} / \mathrm{N}_{o}\right) \\
\text { Signal Link Margin }(\mathrm{SLM})\end{array}$ & $3 \mathrm{~dB}$ \\
Data Rate $(\mathrm{R})$ & $4 \mathrm{bps}$ \\
Transmitter Efficiency $\left(\eta_{T}\right)$ & 0.4 \\
Receiver Efficiency $\left(\eta_{R}\right)$ & 0.6 \\
System Noise Temperature $\left(\mathrm{T}_{s}\right)$ & $75 \mathrm{~K}$ \\
Transmit \& Receive Antenna Pointing Loss $(\mathrm{each})\left(\mathrm{L}_{P L}\right)$ & $-3 \mathrm{~dB}$ \\
Polarization Loss $\left(\mathrm{L}_{P}\right)$ & $-2 \mathrm{~dB}$ \\
Line Loss $\left(\mathrm{L}_{l}\right)$ & $-2 \mathrm{~dB}$ \\
Implementation Loss $\left(\mathrm{L}_{i m p}\right)$ & $-3 \mathrm{~dB}$ \\
Atmospheric Loss $\left(\mathrm{L}_{a t m}\right)$ & $-0.3 \mathrm{~dB}$ \\
\hline Total Losses before Space $/ \mathrm{Noise} \mathrm{Losses})$ & $-10.3 \mathrm{~dB}$ \\
\hline
\end{tabular}

In addition, with the UHF and Stanford SRI case requiring enormous amounts of power, the Deep Space Network is the only ground station option. The $70 \mathrm{~m}$ dishes operate at two RF frequencies, S-Band and X-Band. The DSN also operates at Ka-Band using its $34 \mathrm{~m}$ beam waveguide antenna.

The link analysis uses typical RF communications link equations. These culminate in a variety of equations. The main equation is the signal-to-noise ratio equation shown in Equation 8.1, where $\mathrm{P}_{t}$ is the RF power in Watts, $\mathrm{G}_{t}$ is the transmitter gain in Watts, $\mathrm{G}_{r}$ is the receiver gain in Watts, $k$ is the Boltzmann constant in $\mathrm{J} / \mathrm{K}, \mathrm{T}_{s}$ is the system noise temperature in Kelvin, $\lambda$ is the RF wavelength in meters, $d$ is the distance between the two antennas in meters, and $\mathrm{L}_{T P L}$ and $\mathrm{L}_{R} P L$ is the transmit 
antenna pointing loss and receive antenna pointing loss, respectively, in Watts.

$$
\frac{E_{b}}{N_{o}}=\frac{P_{t} G_{t} G_{r} L_{p} L_{l} L_{a t m} L_{i m p} L_{R P L} L_{T P L}}{k T_{s} R\left(\frac{4 \pi d}{\lambda}\right)^{2}}
$$

The gain for a parabolic dish is calculated using Equation 8.2, where $\eta_{t}$ is the transmitter efficiency and $D_{t}$ is the diameter of the dish in meters.

$$
G_{t}=\frac{\eta_{t} \pi^{2} D_{t}^{2}}{\lambda^{2}}
$$

The receiver gain can be calculated with a very similar equation shown in Equation 8.3, although using the receiving dish parameters.

$$
G_{r}=\frac{\eta_{r} \pi^{2} D_{r}^{2}}{\lambda^{2}}
$$

The equations can be rearranged to solve for required RF power for the various ground station options along the Voyager 1 trajectory. Using the values listed in Table 8.1 and assuming the spacecraft's determined dish size of $7.75 \mathrm{~m}$ in diameter, the required $\mathrm{RF}$ power to maintain a signal to noise ratio of $10 \mathrm{~dB}$ with an added signal link margin of $3 \mathrm{~dB}$ can be calculated as a function of the spacecraft's distance for various ground station solutions. This is shown in Figure 8.1. 


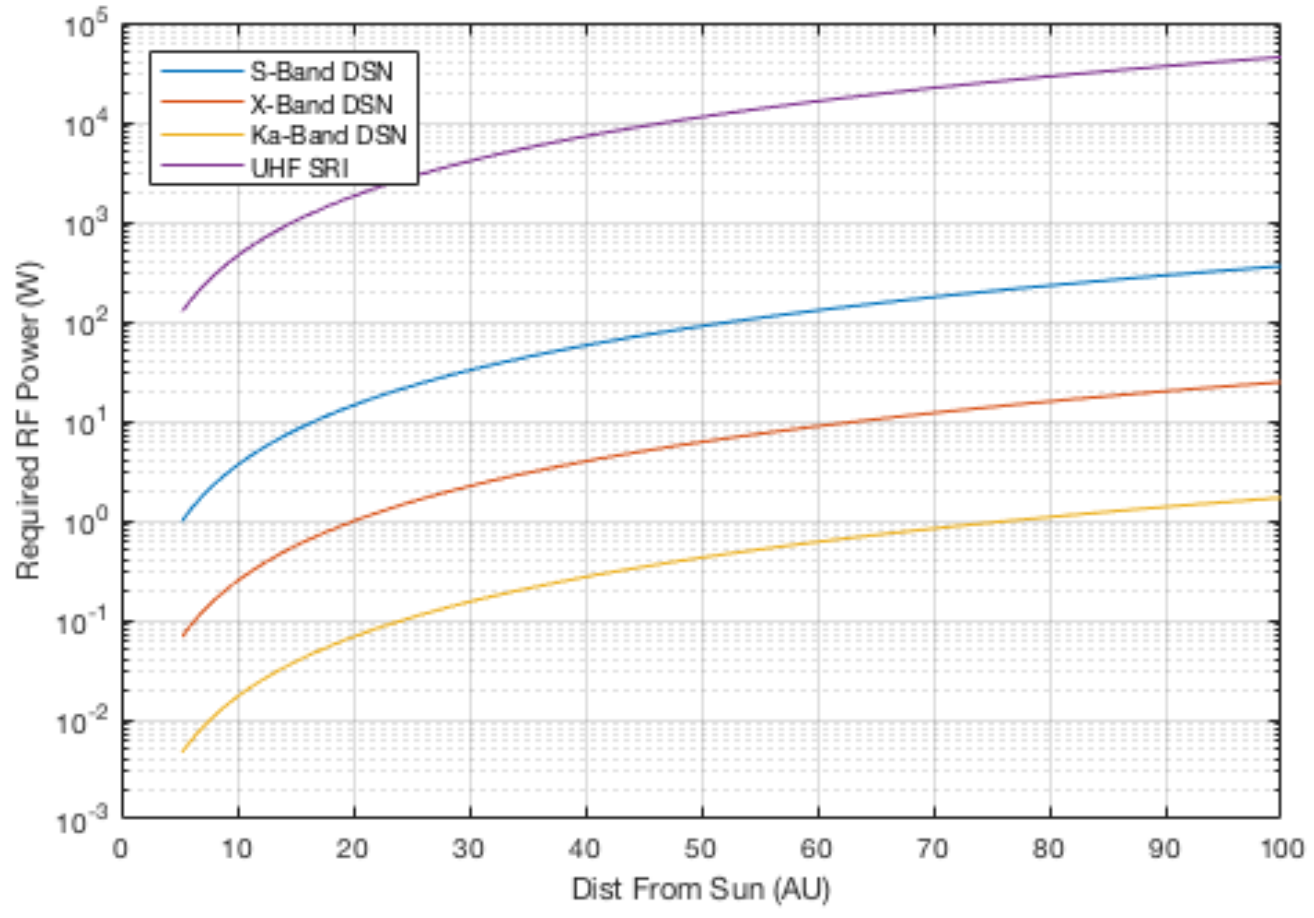

Figure 8.1: RF Power Required for a $7.75 \mathrm{~m}$ Diameter Dish at $10 \mathrm{bps}$ at Various Distances From the Sun

As seen in the curve, X-Band and Ka-Band appear to be the most attractive options once reaching large distances of up to $100 \mathrm{AU}$, requiring the least RF power. However, it should be noted that S-Band can close the link with $1 \mathrm{~W}$ of RF power when near Jupiter. Due to its lower frequency, S-Band will result in a higher beamwidth for the antenna, requiring less maneuvers to track Earth. This may be optimal for the spacecraft's ADCS system when maneuvers are required more frequently.

While Ka-Band appears to be a prime option, high frequencies are highly sensitive to surface irregularities. Because the spacecraft will be using an inflatable dish, there are bound to be surface irregularities. This issue is highlighted in Alessandra Babuscia's paper, in which she details that using Ka-Band with the inflatable antenna will be almost impossible. However, she states that X-Band is indeed feasible given the surface irregularities. Therefore, the spacecraft will need to operate on X-Band 
in order to minimize the required $\mathrm{RF}$ power with the inflatable dish. Therefore, operating on X-Band, the spacecraft will need to generate $23 \mathrm{~W}$ of RF power. The power chapter of this paper determined that the spacecraft will need to transmit once a week to keep the average power consumption low. For this analysis, the data volume transmitted each week will be $1 \mathrm{~KB}$ or 8000 bits. The spacecraft will be using the minimum data rate to keep the electrical power requirement low, and using $10 \mathrm{bps}$, the transmission will need to occur for 800 s to downlink $1 \mathrm{~KB}$. While this appears low, the instruments will be recording data infrequently. While the true required data volume for transmission must be analyzed further, $1 \mathrm{~KB}$ will provide a good reference point for determining requirements down the line.

The transmitter will need to be highly specialized, requiring low standby power consumptions while able to generate over $23 \mathrm{~W}$ of RF power. While the exact specifications of the transmitter should be investigated in further detail, the transmitter will be assumed as a solid state power amplifier (SSPA), a compact and low cost spacecraft amplifier. Typical SSPAs range from 0.5 to $1.5 \mathrm{~kg}$ and can be up to $30 \%$ efficient. The SSPA is assumed to occupy a $1 \mathrm{U}$ volume. The feed for the antenna will be presumed as the patch antenna used in Babuscia's inflatable antenna design. This patch antenna is assumed to be $10 \mathrm{~cm} \mathrm{x} 10 \mathrm{~cm}$ in area.

\subsection{Communications Subsystem Summary}

The communications subsystem characteristics are summarized in this section. This subsystem mainly consists of the SSPA, the patch antenna feed, and the parabolic dish. The main parameters are listed in Table 8.2. 
Table 8.2: Communications Subsystem Characteristics Summary

\begin{tabular}{|c|c|}
\hline Feed & $100 \mathrm{~cm}^{2}$ Patch Antenna \\
Transmitting Dish & $7.75 \mathrm{~m}$ Diameter \\
Ground Station & Deep Space Network 70m Dish \\
Data Rate (R) & $10 \mathrm{bps}$ \\
Frequency at 100 AU & X-Band $(8.4 \mathrm{GHz})$ \\
Transmission Duration & 800 seconds \\
Maximum RF Power & $23 \mathrm{~W}$ \\
Amplifier & $1 \mathrm{~kg} ; 1 \mathrm{U}$ SSPA \\
\hline
\end{tabular}




\section{Chapter 9}

\section{ADCS AND PROPULSION SUBSYSTEM}

During the length of the mission, the spacecraft will need to adjust its pointing in order to track the Earth as it escapes the solar system. Fortunately, active attitude control rates decrease as the spacecraft travels further away from the sun. Nonetheless, tracking the Earth for 30 years can amount to significant propellant and power. The spacecraft will also need to perform a $10 \mathrm{~m} / \mathrm{s}$ delta-V maneuver to separate itself from the original trajectory.

There are limited attitude control options for low power spacecraft. Traditional reaction wheels and control moment gyros often require several Watts of power. While these attitude control systems can be miniaturized for use in nanosatellites, they still require power consumptions on the order of $200 \mathrm{~mW}$ for each wheel[67], drastically increasing the standby power of the spacecraft. A significantly more attractive attitude control option for the small OSS probe would be the use of spin stabilization. Spin stabilization would keep the spacecraft pointing inertially in space and minimize the active attitude control requirements for the ADCS subsystem, thus decreasing the standby power, a critical aspect of the spacecraft. A drawback of using spin stabilization is the difficulty of changing the spin axis and overcoming the gyroscopic resistance. However, with the lack of perturbations in interplanetary space, the spin rate for spin stabilization of the spacecraft can be low, while still maintaining inertial pointing.

In order for spin stabilization to work for the spacecraft, the mass moment of inertia about the spin axis must be the largest mass moment of inertia of the spacecraft. Spin stabilization of the spacecraft will keep the spacecraft inertially pointing during the $10 \mathrm{~m} / \mathrm{s}$ delta-V manuever, minimizing correctional attitude maneuvers during the 
burn, similar to some solid rocket motor designs. In addition, spin stabilization during the undeployed state will allow for ease of deployment of the payload booms and inflatable antenna. For this to be the case, the mass moment of inertia ratio must remain adequate for spin stabilization for both the deployed and undeployed state. This culminates in the configuration design choice of a relatively flat bus, hence the $33 \mathrm{~cm} \times 33 \mathrm{~cm} \times 15 \mathrm{~cm}$ antenna compartment and the $6 \mathrm{U}$ bus attached below it.

Overall, the ADCS and propulsion subsystem must accomplish three tasks. First, it must be able to spin up the spacecraft to its spin stabilization spin rate. Second, it must be able to impart a $10 \mathrm{~m} / \mathrm{s}$ delta- $\mathrm{V}$ maneuver. Third, it must be able to actuate the attitude change maneuvers with enough accuracy for the entire duration of the mission. These three tasks must be consider in order to choose a suitable system.

\subsection{Attitude Control Requirements}

The attitude control requirements must be determined in order to choose and size the ADCS system. Because the spacecraft will be using a high gain antenna with a large diameter dish, the beamwidth of the antenna will be small, on the order of a few tenths of a degree when using X-Band. This will require a significant amount of attitude correction maneuvers in order to point the spacecraft towards Earth as the spacecraft travels along its trajectory and as the Earth orbits around the Sun. Analyzing the trajectory for Voyager 1, the total amount of attitude change as well as the maximum attitude change rates can be determined.

The following graphs show the attitude from the spacecraft to Earth over the length of the Voyager 1 trajectory from Jupiter to 100 AU. This analysis assumes the spacecraft will travel in the ecliptic plane and will only adjust for 2 dimensional attitude corrections. The analysis uses Earth's ephemeris data starting from the Voyager 1 Jupiter encounter around 1979. Figure 9.1 shows the change in attitude if 
the spacecraft were to track Earth along the Voyager 1 trajectory from Jupiter to 100 AU. Figure 9.2 details the attitude angle rates in degrees per year during the same trajectory. The wobbles shown in the figure are a result of the Earth orbiting the Sun as the spacecraft travels along its trajectory.

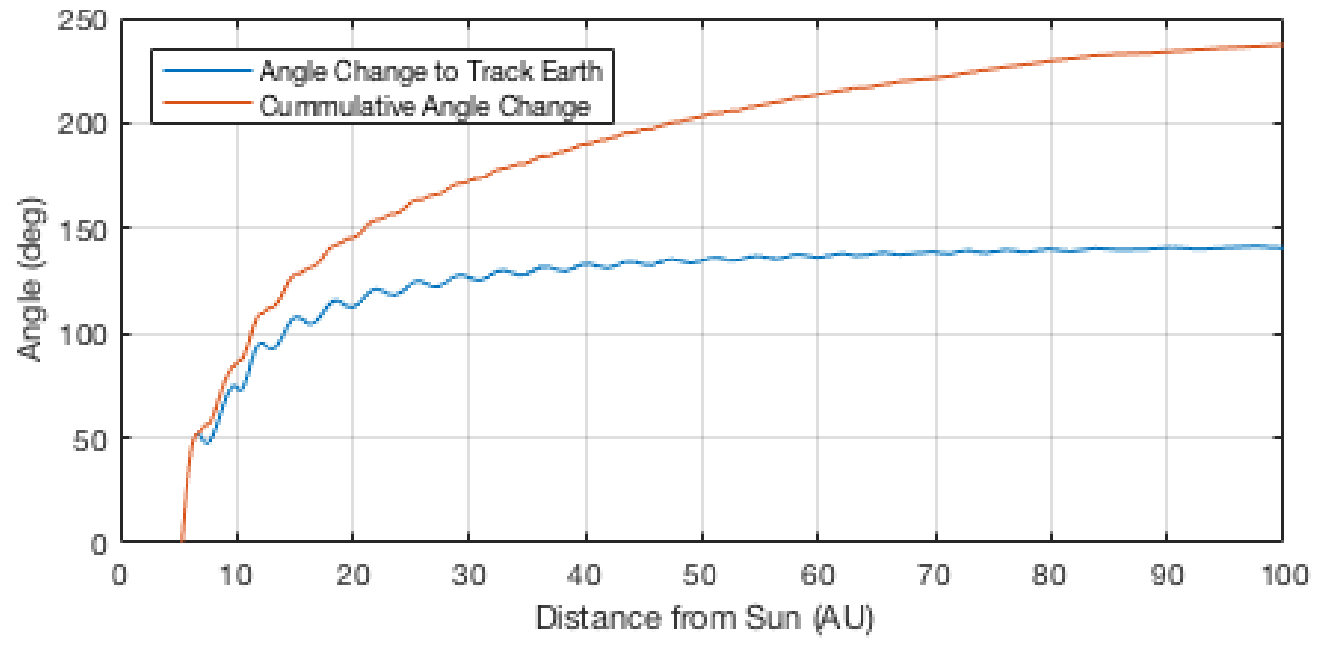

Figure 9.1: Attitude Angle Change During Voyager 1 Trajectory From Jupiter to 100 AU

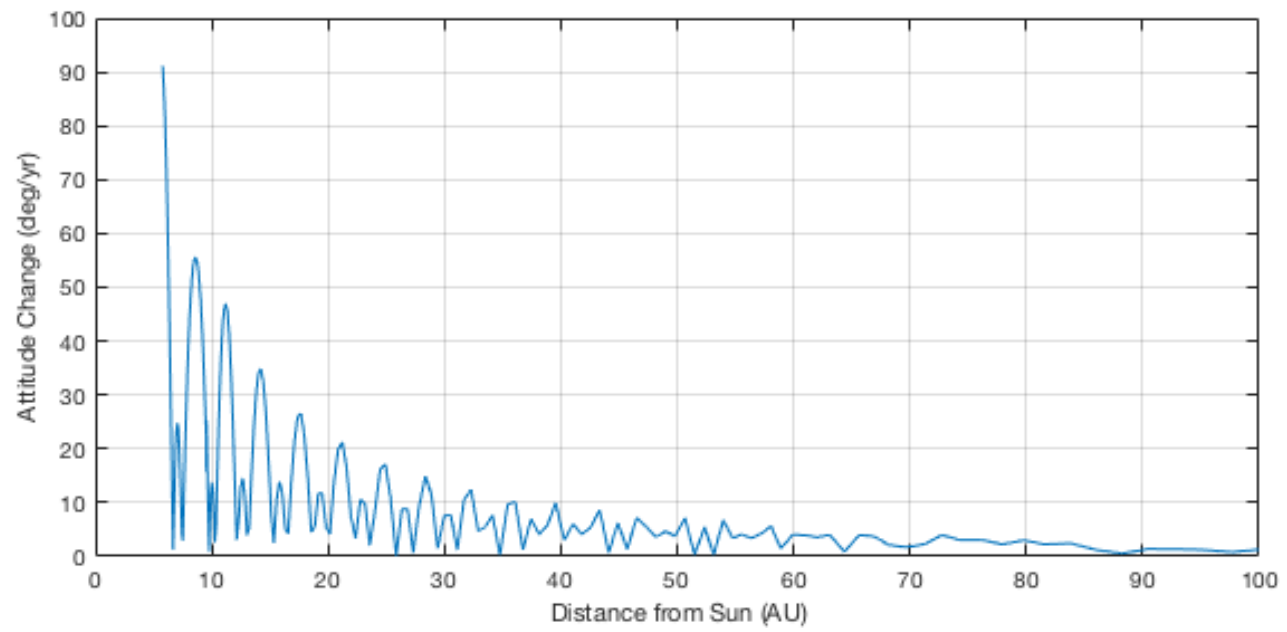

Figure 9.2: Attitude Rates During Voyager 1 Trajectory From Jupiter to $100 \mathrm{AU}$

Although the total angle displacement only reaches under 150 degrees, the spacecraft will need to account for the attitude changes in the reverse direction that cause 
the valleys seen in the blue line in Figure 9.1. The second curve in Figure 9.1 takes the absolute value of the differences in attitude angle from the blue curve, resulting in a total attitude angle change of roughly 240 degrees. However, it should be noted in Figure 9.1 and Figure 9.2 that attitude rates and attitude angle changes drop significantly as the probe reaches $100 \mathrm{AU}$. The earlier portions of the trajectory will require the most active attitude control.

In order to determine an adequate actuator to perform these attitude changes, we can use Equation 9.1, which comes from a presentation by Olivier L. de Weck from the Department of Aeronautics and Astronautics at Massachusetts Institute of Technology.

$$
\Delta \theta \approx \frac{r F \Delta t}{I \omega}
$$

$\Delta \theta$ represents the spin axis attitude change of the spacecraft as a function of the moment arm, $r$, the force imparted on the moment arm, $F$, the length of time that the force is imparted, $\Delta t$, the moment of inertia of the axis of which the spacecraft will be rotated about, $I$, and the spin rate of the space craft, $\omega$. It should be noted that imparting a force to change the spin axis will induce a precession, resulting in the spacecraft "wobbling" about its spin axis. However, nutation dampers in the form of propellant sloshing or a dedicated viscous ring damper can settle the spacecraft wobble onto the intended spin axis. Nonetheless, Equation 9.1 can be used to calculate and quantify the necessary impulse or force to fulfill the attitude change requirements.

\subsubsection{Calculating Mass Moment of Inertia}

A mass moment of inertia must be assumed for both the deployed and undeployed spacecraft to determine the its resistance to torque during attitude change maneuvers. The components' shape approximations can be seen in Figure 9.3. The figure 
also shows the spin axis for spin stabilization around the Z-axis. The undeployed spacecraft shows the antenna compartment, housing the solar panel face, reflector dish, and the unfurlable side radiator panels.
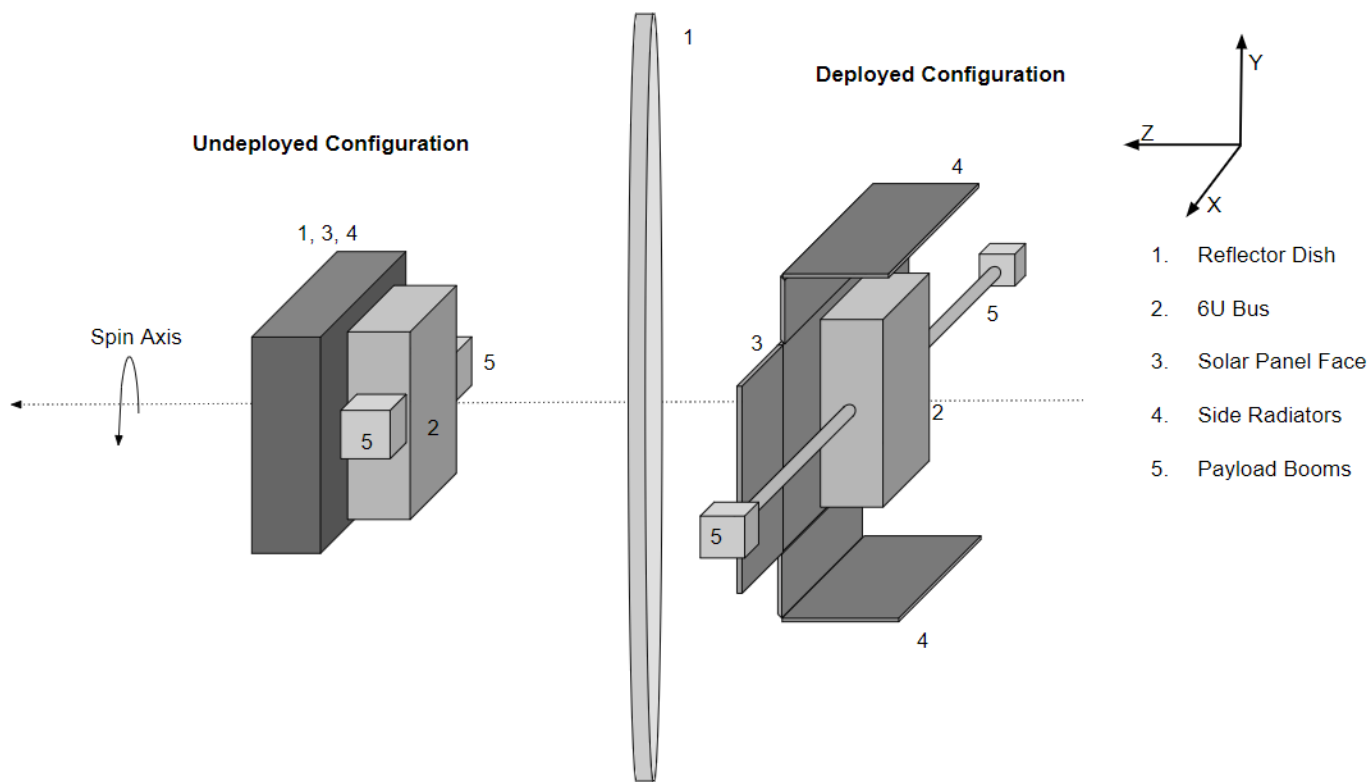

Figure 9.3: Spacecraft Configuration Geometry Approximations for Deployed and Undeployed State

Assuming a $13 \mathrm{~kg}$ deployable antenna with a diameter of $7.75 \mathrm{~m}$, we can approximate this as a thin disk. This thin disk will be placed at the midpoint of the dish depth. The bus section of the spacecraft can be approximated as a $30 \mathrm{~cm} \times 20 \mathrm{~cm}$ x $10 \mathrm{~cm}$ rectangular prism with a mass of $10 \mathrm{~kg}$. The front panel consisting of the feed and solar panels will be approximated as a $33 \mathrm{~cm}$ x $33 \mathrm{~cm}$ flat plane. The side radiators can be approximated similarly to the front panel, although in a different position and half the size. The side panels rest $31.5 \mathrm{~cm}$ away from the spin axis. The final component of the spacecraft would consist of the two deployable booms with the magnetometer and plasma instrument on either end. This can be approximated as a slender rod extending $0.25 \mathrm{~m}$ on either end of the maximum radius of the dish for a total length of $8.25 \mathrm{~m}$ and a mass of $2 \mathrm{~kg}$. With a focal length of $2.10 \mathrm{~m}$ and a dish 
depth of $1.79 \mathrm{~m}$, the distance from the solar panel and feed face to the position of the thin disk approximation can be calculated to be $1.2 \mathrm{~m}$. The bus is assumed to rest $3 \mathrm{~cm}$ away from the back of the solar panel and feed face. These relative positions can be seen in Figure 9.4. In the figure, the payload booms extend in and out of the page.

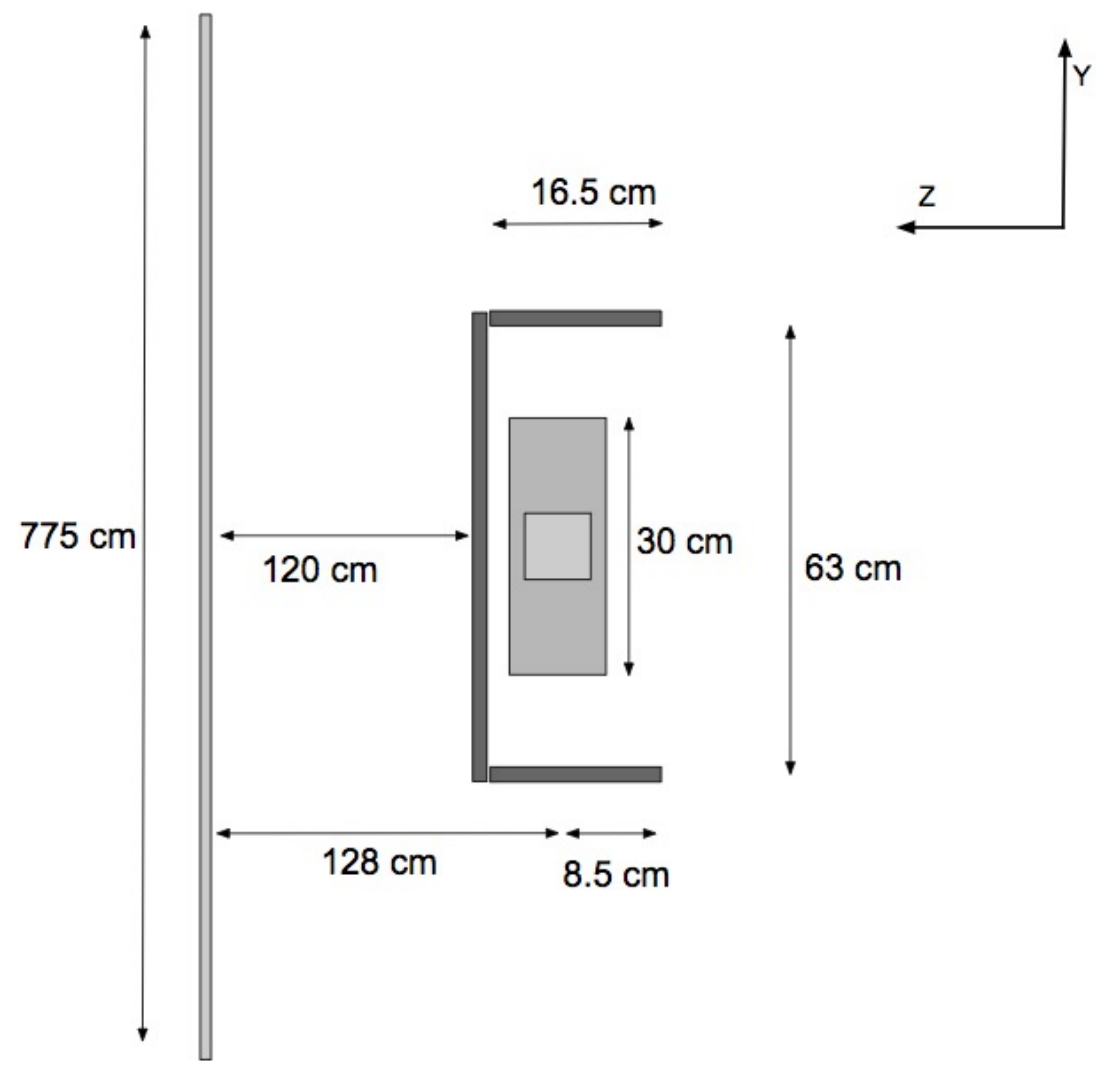

Figure 9.4: Spacecraft Configuration Relative Positions

Using approximate shapes and masses, the mass moment of inertias (MMIs) can be approximated. These parameters are layed out in Table 9.1.

Using standard mass moment of inertia equations as well as the parallel axis theorem equation, Equation 9.2, the mass moment inertias for the spacecraft can be calculated. The results of these calculations can be seen in Table 9.1. 
Table 9.1: Deployed Spacecraft Components and Geometric Approximations

\begin{tabular}{|c|c|c|c|}
\hline Component & Mass & Dimensions & Geometric Approximation \\
\hline Inflatable Antenna & $13 \mathrm{~kg}$ & $\mathrm{D}=7.75 \mathrm{~m}$ & Thin Disk \\
Bus & $10 \mathrm{~kg}$ & $30 \mathrm{~cm} \times 20 \mathrm{~cm} \times 10 \mathrm{~cm}$ & Rectangular Prism \\
Main Plate & $1 \mathrm{~kg}$ & $33 \mathrm{~cm} \times 33 \mathrm{~cm}$ & Thin Plate \\
Side Radiators & $0.72 \mathrm{~kg}$ each & $33 \mathrm{~cm} \mathrm{x} 16.5 \mathrm{~cm}$ & Thin Plate \\
Payload Booms & $2 \mathrm{~kg}$ & $\mathrm{~L}=8.25 \mathrm{~m}$ & Slender Rod Through Axis \\
\hline
\end{tabular}

$$
I=I_{c m}+m * d^{2}
$$

\section{Table 9.2: Deployed Spacecraft Mass Moment of Inertias}

\begin{tabular}{|c|c|}
\hline Axis & Mass Moment of Inertia $\left(\mathrm{kg} \mathrm{m}^{2}\right)$ \\
\hline X-Axis & 59.87 \\
Y-Axis & 71.18 \\
Z-Axis & 109.14 \\
\hline Center of Mass & $69.82 \mathrm{~cm}$ along Z-Axis \\
\hline
\end{tabular}

The mass moment of inertia results show that the largest principal axis is the Z-axis, the intended spin stabilization axis. Because the mass moment of inertia is larger than the $\mathrm{X}$ and $\mathrm{Y}$-axis, this will ensure that the spacecraft remains spinning around the intended axis while deployed.

We can now estimate the undeployed mass moment of inertias of the spacecraft to analyze whether the configuration will remain gyroscopically stable around the intended axis. The spacecraft components and geometric approximations for the undeployed state can be seen in Table 9.3.

Several components are lumped into one approximation for their mass moment of inertias. The inflatable antenna compartment is assumed to house the inflatable 
Table 9.3: Undeployed Spacecraft Components and Geometric Approximations

\begin{tabular}{|c|c|c|c|}
\hline Component & Mass & Dimensions & Geometric Approximation \\
\hline Inflatable Antenna Compartment & $15 \mathrm{~kg}$ & $33 \mathrm{~cm} \mathrm{x} 33 \mathrm{~cm} \times 15 \mathrm{~cm}$ & Rectangular Prism \\
Bus & $12 \mathrm{~kg}$ & $30 \mathrm{~cm} \mathrm{x} 20 \mathrm{~cm} \times 10 \mathrm{~cm}$ & Rectangular Prism \\
\hline
\end{tabular}

antenna, while the side radiators and main plate make up the walls of the compartment. The bus and payload booms are lumped into one approximation, adding the $2 \mathrm{~kg}$ to the bus mass. Once again, the mass moment of inertias in the principal axes can be approximated for this composite shape. The results of the calculations are shown in Table 9.4 .

Table 9.4: Undeployed Spacecraft Mass Moment of Inertias and CM

\begin{tabular}{|c|c|}
\hline Axis & Mass Moment of Inertia $\left(\mathrm{kg} \mathrm{m}^{2}\right)$ \\
\hline X-Axis & 0.42 \\
Y-Axis & 0.37 \\
Z-Axis & 0.40 \\
\hline Center of Mass & $18.1 \mathrm{~cm}$ along Z-Axis \\
\hline
\end{tabular}

The undeployed configuration of the spacecraft results in the largest moment of inertia principal axis of the $\mathrm{X}$-axis. While the mass moment of inertia for the Z-axis is not significantly different from the X-axis. This will mean that the spacecraft will naturally want to rotate around the $\mathrm{X}$-axis. However, because the spacecraft will not remain in its undeployed state for long, the difference in mass moment of inertia can be neglected for this short portion of the mission. Any perturbations can be corrected with a sufficient amount of thrusters. 


\subsubsection{Calculating Spin Up Impulse Requirement}

Now that an approximate mass moment of inertia has been calculated, the required spin rate of the undeployed spacecraft and thus required impulse can be calculated to attain a suitable spin rate when the spacecraft has fully deployed its antenna and payload booms. This spacecraft will likely need to spin itself up without aid from its deployment system. Torque is related to angular acceleration by Equation 9.3.

$$
T=I \alpha
$$

The torque equation can be easily manipulated into solving for the impulse required to reach a spin rate by multiplying both sides by time, as shown in Equation 9.4, where $r$ is the moment arm, $\omega$ is the spin rate in radians, and $I$ is the mass moment of inertia.

$$
\text { Impulse }=\frac{I \omega}{r}
$$

Selecting the deployed mass moment of inertia about the Z-axis of $109.14 \mathrm{~kg} \mathrm{~m}^{2}$, a spin rate of $2 \mathrm{RPM}$, equivalent to $0.21 \mathrm{rads} / \mathrm{sec}$, and a moment arm of $13 \mathrm{~cm}, 2 \mathrm{~cm}$ shy of the largest possible moment arm of a $6 \mathrm{U}$ bus, the total impulse required to reach 2 RPM is just under 176 Ns. It should be noted that the equivalent angular momentum of the deployed configuration at spin rate of $2 \mathrm{RPM}$ is the same as the undeployed configuration at a spin rate of 542 RPM. Although this is an incredibly fast spin rate, the spacecraft could gain angular momentum over time, spinning up to an initial spin rate sufficient for the $10 \mathrm{~m} / \mathrm{s}$ delta-V maneuver, subsequently deploying the antenna and payload booms, and finally performing the final spin up maneuvers to reach the intended spin rate at the deployed state. However, because the spacecraft will likely induce a torque on the system when the mechanisms are deployed, the force imparted 
on the lightweight payload booms must be considered when sizing the actuator for the spacecraft.

\subsection{Actuation Options}

Several actuation methods for changing the spacecraft's attitude were investigated, including photonic laser thrusters (PLTs), electro-chromatic LCD panels, and traditional thruster systems.

\subsubsection{Photonic Laser Thrusters}

Photonic laser thrusters have the potential to generate thrust at high specific impulses as well as open up the possibility of reaching speeds up to a fraction of the speed of light. This type of thruster utilizes the momentum of a photon to propel itself, similar to a solar sail. The thruster works by bouncing high power lasers between a ground-based or space-based source and the spacecraft. Young K. Bae has been exploring this concept as well as adding an amplifying aspect to the laser reflection[68]. While the spacecraft is not looking to significantly increase its speed, small attitude changes over large amounts of time using a PLT could successfully track Earth. The issue with this thruster is that the PLT requires lots of electrical power and accurate

pointing if the spacecraft is to receive the laser power from a source near Earth. The PLT would require a substantial and expensive ground or space-based laser source that could be replaced with traditional attitude control systems for much lower cost. Alternatively, the OSS probe could possibly house a PLT onboard. Pointing the PLT in a desired direction, the spacecraft could use the action and reaction forces from the photons leaving the light source to change its attitude. Unfortunately, using simple calculation using the available power, the thrust imparted by the PLT is several orders of magnitude too small for the required attitude change requirements. In addition, 
the PLT would require excessive amounts of electrical power for long lengths of time, draining the little available power to the spacecraft necessary for bus electronics and high power processes. The technology is also severely under-developed and is far from flight ready.

\subsubsection{IKAROS Electro-Chromatic LCD Panels}

IKAROS was a spacecraft flown by the Japanese space agency (JAXA) and was the first spacecraft to successfully demonstrate solar sail technology. The spacecraft was a $300 \mathrm{~kg}$ spacecraft with a $14 \mathrm{~m} \times 14 \mathrm{~m}$ solar sail that was used to maneuver the spacecraft over time and drop the perihelion of its orbit in order to reach Venus. The

primary mission was declared a success when IKAROS flew by Venus in December of $2010[31]$.

A particularly intriguing aspect of IKAROS' solar sail was its use of eighty LCD panels used for attitude control. These LCD panels can change the optical properties with small amounts of electrical power, effectively controlling the amount of photonic impulse imparted on the surface. Figure 9.5 shows a photograph of the change in specular reflectivity of the surface when turned on and turned off. 


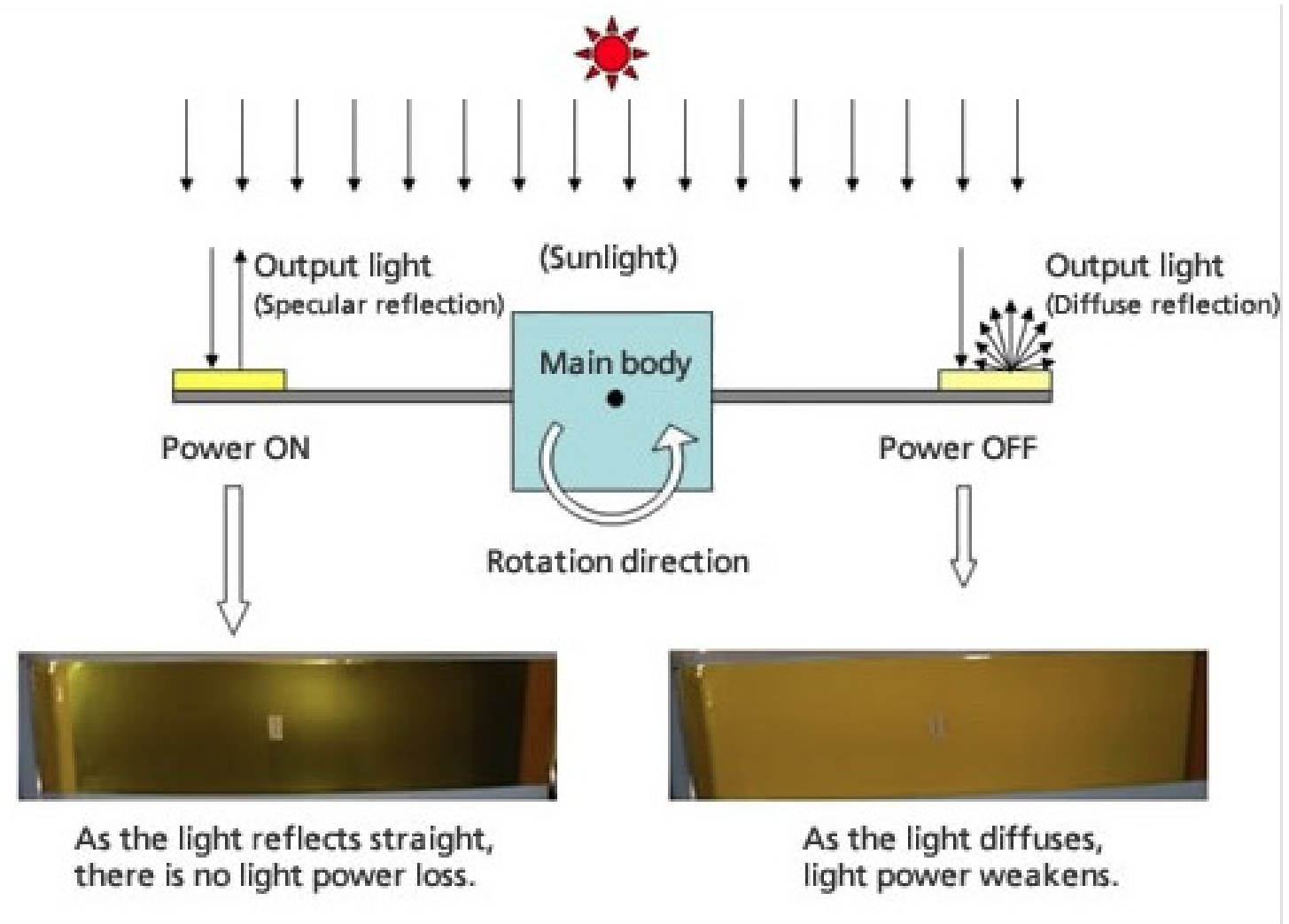

Figure 9.5: IKAROS Attitude Control LCD Panels[11]

With a spin stabilized spacecraft, the LCD panels could be cycled to provide a constant torque in a desired direction. The panels would be a very effective low power solution for attitude maneuvers along the trajectory. With a large diameter dish, the LCD panels may be placed on the edge of the reflector to provide a large moment arm to induce a torque on the spacecraft. A quick calculation can be performed to determine if this method is viable for the mission of the spacecraft. Selecting one of the attitude rates from the reference Voyager 1 mission, this attitude change rate can result in a required LCD panel area.

Observing the curve in the Figure 9.2, attitude rates start at 90 degrees per years. However, scientific measurements inside 40 AU are not particularly useful in describing the OSS environment. The desirable OSS data would be measurements towards 
the end of the mission as it near the termination shock region at $100 \mathrm{AU}$. Unfortunately for this method of actuation, solar intensity is a large factor in providing the necessary force to change the attitude of the spacecraft. However, it may be possible to use these LCD panels when closer to the sun, reducing the amount of fuel that would be necessary for thrusters when the solar intensity diminishes. For these reasons, we will investigate the LCD panel area requirement for attitude changes after 40 AU. The attitude rates shown in Figure 9.2 spike to a maximum of just under 10 degrees per year at roughly $43 \mathrm{AU}$. This is equivalent to $3.17 \mathrm{E}-7$ degrees per second with a corresponding solar intensity of $0.739 \mathrm{~W} / \mathrm{m}^{2}$ at this distance. With an estimated mass moment of inertia about the $\mathrm{X}$ and $\mathrm{Y}$ axis, the required LCD panel area can be calculated.

For the purposes of this analysis, the LCD panel area will be assumed to have perfect specular reflectivity, meaning photons reflect perfectly and impart maximum momentum onto the moment arm. With our $7.75 \mathrm{~m}$ dish, the moment arm translates to $3.875 \mathrm{~m}$. The solar radiation pressure can be calculated with Equation 9.5, where $I_{s}$ is the solar intensity in $\mathrm{W} / \mathrm{m}^{2}, c$ is the speed of light, and $P$ is in Pascals or $\mathrm{N} / \mathrm{m}^{2}$.

$$
P=\frac{I_{s}}{c}
$$

Assuming a low spin rate of 2 RPM, Equation 9.1 can be used to determine the required force to change the spin axis by 3.17E-7 degrees in one second. The mass moment of inertia will be assumed as the average between the mass moment of inertia about the $\mathrm{X}$-axis and the $\mathrm{Y}$-axis, as the LCD panels would be place around the circumference of the parabolic dish. Equation 9.1 produces a required force of 2.24E-8 N. Solving for the required area using Equation 9.5, the dish would need approximately $9.08 \mathrm{~m}^{2}$ of LCD panel area at the edge of the dish at the maximum moment arm point. While this value does not appear to completely throw out the 
possibility of using this technology, there are many inefficiencies that would drive this area to infeasible sizes. The dish shape is rather deep, with a maximum angle, thet $a_{o}$, from the edge of the dish to the focal point of 85 degrees. For solar radiation pressure, this means the force would have a large cosine loss factor as the photonic impulse would not perfectly impart its momentum in the desired direction. In addition, although the diffuse reflection from the LCD panels on the other end would result in a weaker light power, it would counteract much of the force applied on the LCD panels on the other end of the dish. It should also be noted that adding significant LCD panel area to the dish would add to the total mass of the system, further increasing the mass moment of inertia and the resulting resistance to torque.

While this exciting LCD panel technology appears perfectly suited for this low power mission, the low solar intensities in deep space result in too little force to sufficiently actuate the spacecraft.

\subsubsection{Traditional Thruster Systems}

Without the possibility of using photonic laser thrusters or LCD panels, actuation options for this mission are reduced to thruster systems. While thruster systems may increase the mass and volume requirements of the spacecraft, the flight heritage and high impulse of these actuation systems result in a robust actuation option for this spacecraft.

There are many thruster systems available for spacecraft and many more under development. There are several considerations that must be taken into account for selecting the appropriate thruster system for this spacecraft and its mission. The spacecraft relies on low power consumption, so the thruster system will need to consume negligible power during standby modes and have a lower energy consumption when performing impulsive maneuvers. Due to low power availability during the mis- 
sion, the propellant will need to be able to survive low temperatures, as heating power will be a scarce resource when nearing distances of $100 \mathrm{AU}$.

\subsection{Thruster Requirements and Trade Study}

Before selecting the thruster system, impulse requirements must be determined for a thruster system placed in the $6 \mathrm{U}$ bus. Assuming the spacecraft follows the Voyager 1 trajectory after the Jupiter flyby, the spacecraft will need to change its attitude a total of 240 degrees, as can be seen in Figure 9.1. Using Equation 9.1, the total required impulse for the system can be calculated as a function of moment arm of the thruster and spin rate. The results of these calculations are shown in Figure 9.6. The required impulse is analyzed for spin rates of 1 to $3 \mathrm{RPM}$ and moment arms of 5 to $20 \mathrm{~cm}$.

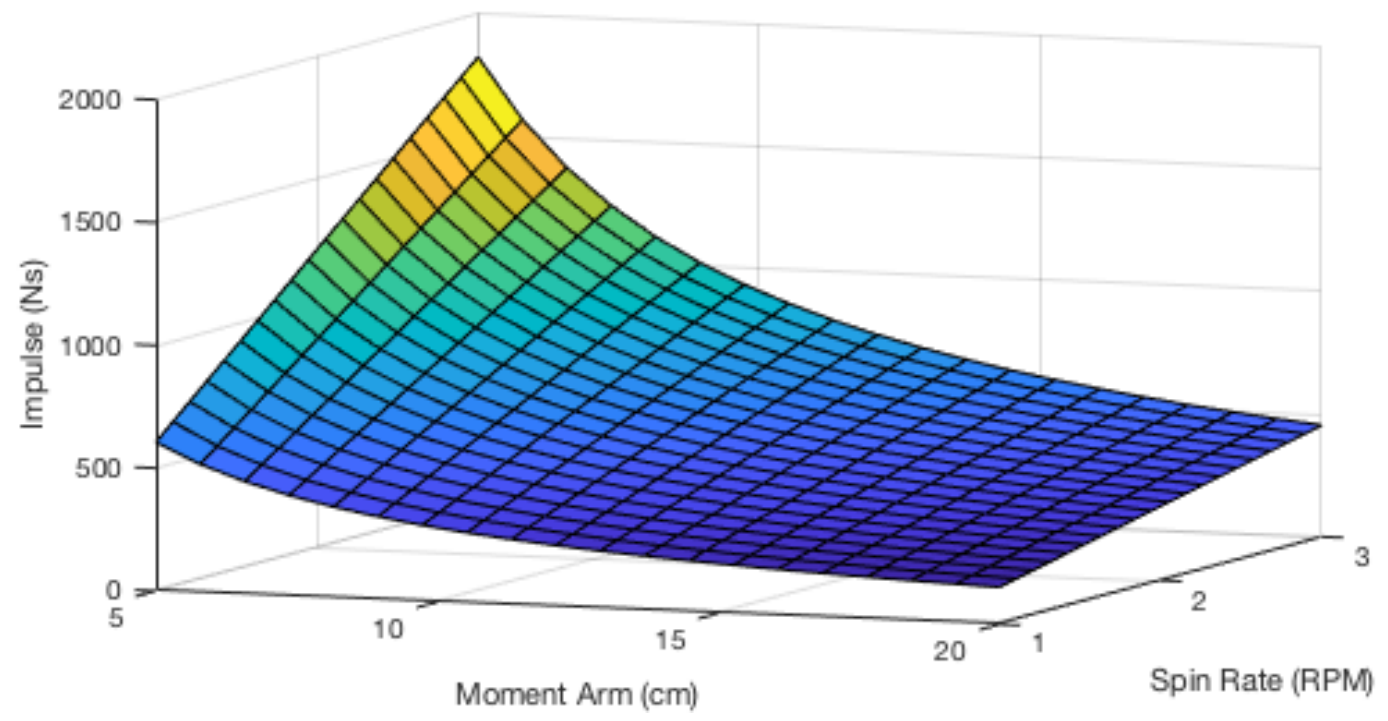

Figure 9.6: Total Impulse Required for Tracking Earth Along Voyager 1 Trajectory as a Function of Moment Arm and Spin Rate

The curve in Figure 9.6 shows that with ample moment arm and low spin rates, the required impulse to track Earth is not unreasonably high. On top of this impulse, 
the $10 \mathrm{~m} / \mathrm{s}$ delta- $\mathrm{V}$ maneuver will require another $300 \mathrm{Ns}$ of impulse, assuming a $30 \mathrm{~kg}$ spacecraft. In order to determine the optimal thruster system to deliver this impulse, a trade can be conducted on various currently available nano-satellite thruster systems.

The total impulse requirement of the ADCS and propulsion system can be detailed for a thruster system positioned in the $6 \mathrm{U}$ bus. Assuming a 2 RPM spin rate, a main thruster moment arm of $13 \mathrm{~cm}$, and a spin up thruster moment arm of $13 \mathrm{~cm}$, the total required impulse to perform all necessary impulsive maneuvers can be seen in Table 9.5.

Table 9.5: Impulse Requirements for All ADCS and Propulsion Ma
\begin{tabular}{|c|c|}
\hline Maneuver & Impulse Requirement (Ns) \\
\hline Spin Up to 2 RPM & 176 \\
$10 \mathrm{~m} / \mathrm{s}$ Orbital Manuever & 300 \\
Earth Tracking Attitude Changes & 404 \\
\hline Total & 880 \\
\hline
\end{tabular}

The thruster system must be capable of $880 \mathrm{Ns}$ of total impulse to satisfy a Voyager 1-like mission. This requirement will be kept in mind when selecting a suitable thruster system for the spacecraft.

The optimal thruster system will need to have high enough thrust to perform the $10 \mathrm{~m} / \mathrm{s}$ burn and perform attitude change maneuvers in a relatively short amount of time to minimize power consumption. On the other hand, the thruster cannot be oversized and produce too high of a minimum impulse to accurately point the high gain antenna towards Earth. On top of this, the propellant of the thruster should not occupy too large a volume, as there are size constraints on the spacecraft. Due to the low power availability during the mission, electric propulsion (EP) systems will not be considered in this trade due to the fact that the spacecraft would need to operate these relatively high power thrusters for large periods of time to provide 
adequate actuation of the spacecraft, resulting in high amounts of power. Although EP thrusters significantly outperform traditional thruster systems in terms of specific impulse, power is at a higher premium than propellant mass and volume.

There are several types of thrusters other than EP that can satisfy the ADCS and propulsion requirements. Currently available thruster systems for the various types of thrusters have been placed into a trade to give an idea of what type of thruster would be optimal for the spacecraft. This can be seen in Figure 9.7.

\begin{tabular}{|c|c|c|c|c|c|}
\hline \multicolumn{6}{|c|}{ PROPULSION TRADE } \\
\hline & & $\begin{array}{c}\text { Busek } 100 \mathrm{mN} \text { HAN } \\
\text { Monoprop HPGP }\end{array}$ & \begin{tabular}{c|c} 
Busek \\
Micro-Resistojet \\
\end{tabular} & VACCO PUC & $\begin{array}{l}\text { VACCO } \\
\text { ArgoMoon } \\
\end{array}$ \\
\hline \multicolumn{2}{|c|}{ Thruster Type } & Mono Propellant & Resistojet & Warm Gas & $\begin{array}{l}\text { Cold Gas \& } \\
\text { Mono Prop }\end{array}$ \\
\hline & Weights (1-4) & & & & \\
\hline Max Thrust & 2 & 4 & 2 & 1 & 4 \\
\hline Min Impulse Bit & 2 & 2 & 5 & 5 & 2 \\
\hline Operating Power & 3 & 3 & 2 & 2 & 2 \\
\hline Standby Power & 4 & 4 & 4 & 4 & 1 \\
\hline Total Impulse & 3 & 3 & 2 & 4 & 5 \\
\hline \multirow[t]{2}{*}{ Min Prop Temp } & 4 & 5 & 2 & 2 & 5 \\
\hline & Score & 66 & 50 & 54 & 57 \\
\hline \multicolumn{2}{|c|}{ Final Choice } & \multicolumn{4}{|c|}{ Busek $100 \mathrm{mN}$ HAN MonoProp HPGP } \\
\hline
\end{tabular}

\section{Figure 9.7: Thruster Trade Study}

The trade study shown in Figure 9.7 weighs several key parameters against each other for four currently available thruster systems. All four of the thruster systems chosen are roughly $1 \mathrm{U}$ in volume in order to accurately compare performance. Four types of thrusters were compared: a mono propellant thruster, a resistojet, a warm gas system, and a hybrid of cold gas and mono propellant. Some of the most important factors in the trade were standby power and minimum propellant temperature. These were heavily weighed as the spacecraft will have very low available power to operate these components on standby power as well as to keep the propellant at an 
operable temperature. Ideally, the spacecraft would not need to divert any electrical power towards heaters or standby power. The ideal propellant choice should be able to reach temperatures of below $0{ }^{\circ} \mathrm{C}$. While the VACCO ArgoMoon hybrid thruster shows promise in its thrust and total impulse values, the $1 \mathrm{~W}$ standby power for the thruster system more than doubles the total standby power consumption for the system[61]. The Busek resistojet and VACCO PUC warm gas thruster options simply had too low of thrust to deliver the necessary impulse without consuming large amounts of power[69][70]. The Busek $100 \mathrm{mN}$ thruster has an acceptable balance of thrust performance, standby power consumption, and minimum propellant temperature. This thruster is a high performance green propellant (HPGP) thruster using AF-M315E propellant, also called Air Force green propellant. This type of propellant is known to be able to survive very low temperatures of up to $-80^{\circ} \mathrm{C}$, making it ideal for long duration interplanetary cruises[63]. The major parameters of this thruster are shown in Table 9.6.

Table 9.6: Busek BGT-X1 100mN Green Propellant Thruster Parameters

\begin{tabular}{|c|c|}
\hline Nominal Thrust & $0.1 \mathrm{~N}$ \\
Throttleable Range & $20-180 \mathrm{mN}$ \\
Vaccuum Specific Impulse & $214 \mathrm{~s}$ \\
Minimum Impulse Bit & $<14 \mathrm{mNs}$ \\
Pre-Heat Power & $0.38 \mathrm{Whr}$ \\
Operating Power & $4.5 \mathrm{~W}$ \\
Total Impulse & $550 \mathrm{Ns}$ \\
Mass & $1.5 \mathrm{~kg}$ \\
Volume & $1 \mathrm{U}$ \\
\hline
\end{tabular}

A single BGT-X1 thruster system produces $550 \mathrm{Ns}$ of impulse[71], so it is clear that the spacecraft will need house two of these systems to provide sufficient impulse 
for the entirety of the mission. The spacecraft will also require a variation of this thruster with an extra nozzle to perform the spin up maneuvers. A simple graphic of the thruster configuration is shown in Figure 9.8.

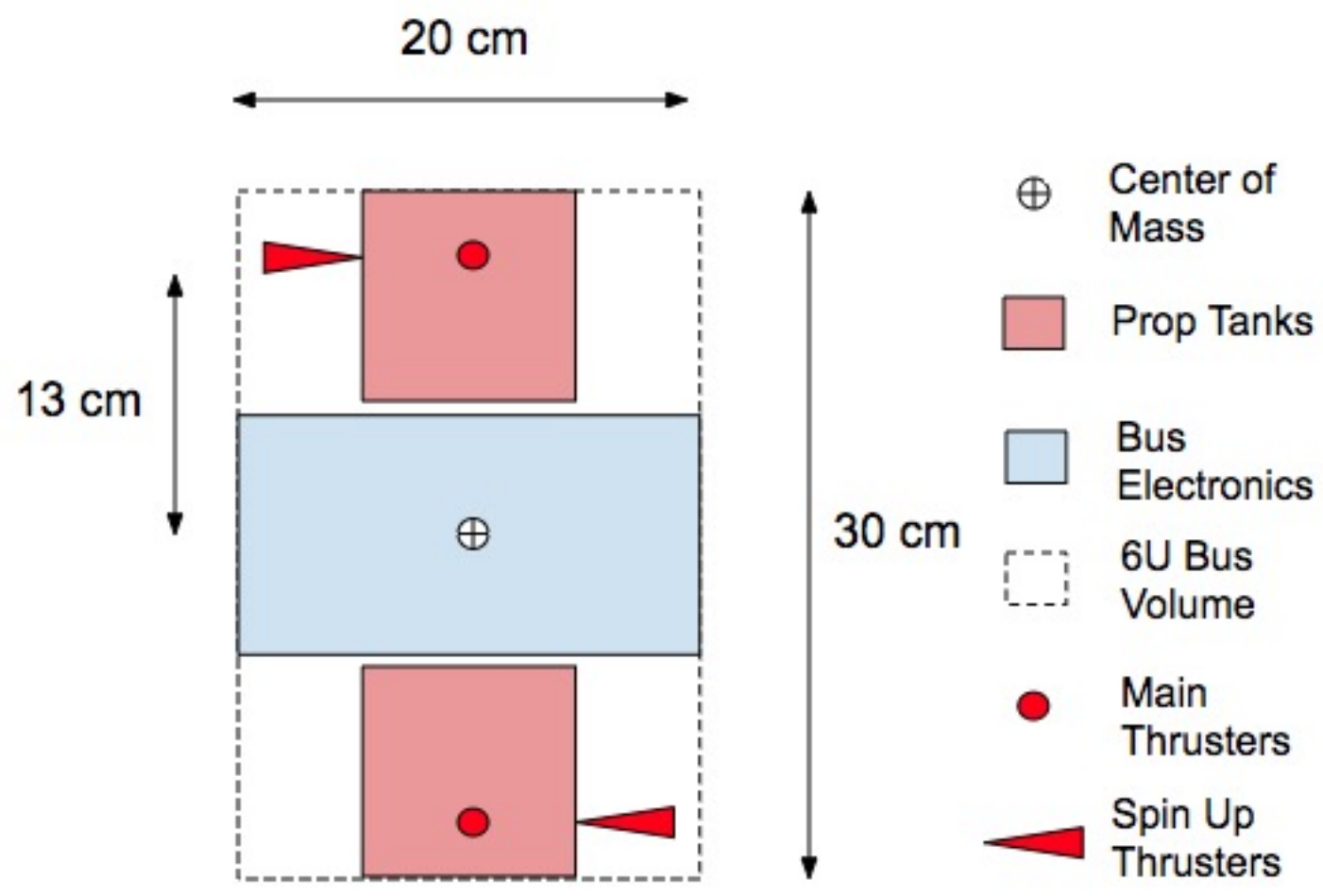

Figure 9.8: Possible Thruster Configuration in Bus

The main thrusters, shown as the red dots, are responsible for performing the 10 $\mathrm{m} / \mathrm{s}$ delta- $\mathrm{V}$ maneuver as well as induce precession on the spin stabilized spacecraft to change its spin axis. Due to possible inaccuracies of the center of mass, the spacecraft may need 4 main thrusters to control rotation rates about its $\mathrm{X}$ and $\mathrm{Y}$-axis.

Using the S-Band and X-Band beamwidths from the $7.75 \mathrm{~m}$ diameter reflector dish, the amount of maneuvers and frequency of maneuvers can be approximated. These beamwidths are 1.23 and 0.322 degrees for $\mathrm{S}$ and X-Band, respectively. As noted previously, the beamwidths of the antenna will be small and drive the number and frequency of maneuvers, especially when using X-Band. A simple analysis 
can be performed on the Voyager 1 trajectory to estimate the necessary spacecraft maneuvers when using thrusters. The following analysis propagates the Voyager 1 trajectory and counts the number of attitude corrections to keep the Earth within the beamwidth of the high gain antenna. Figure 9.9 shows the amount of maneuvers and the frequency of maneuvers required when using the S-Band beamwidth. Figure 9.10 shows the amount and frequency of attitude correction maneuvers when using the X-Band beamwidth.

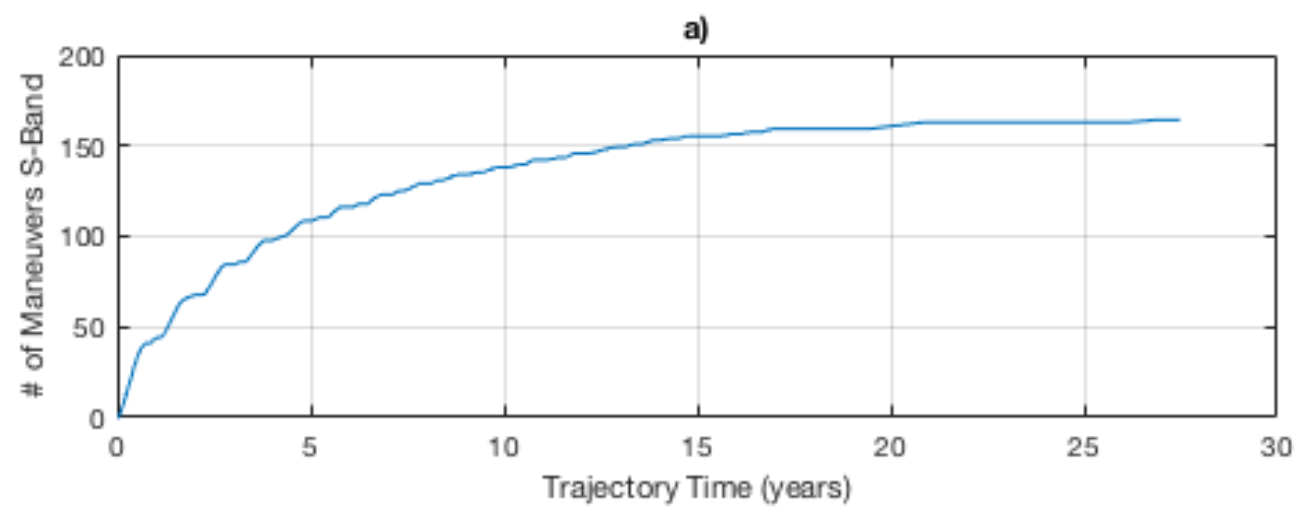

b)

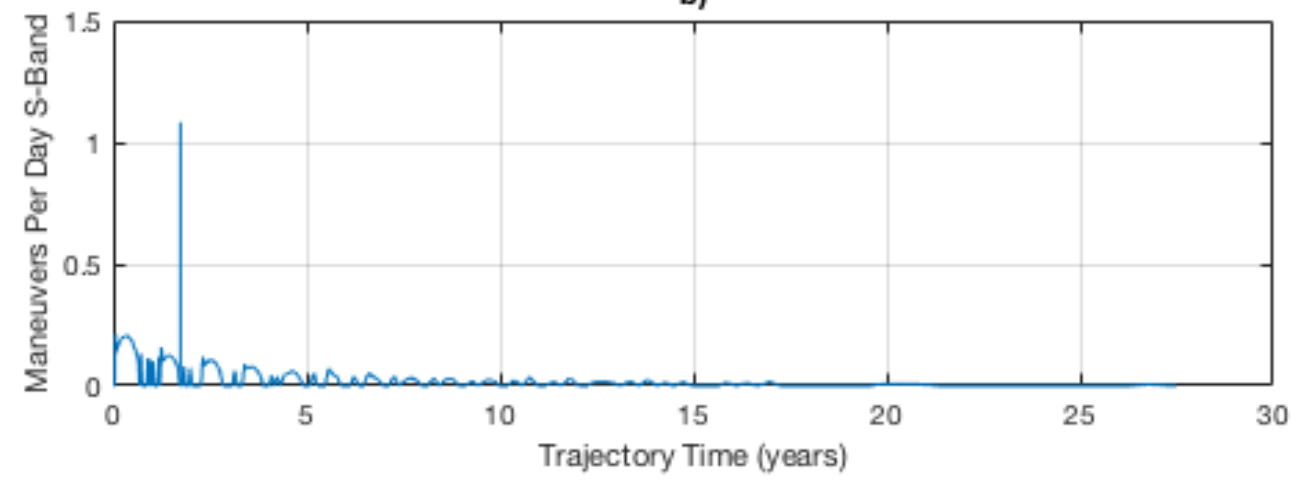

Figure 9.9: a) Number of Required Attitude Correction Maneuvers Using S-Band Beamwidth; b) Frequency of Maneuvers 


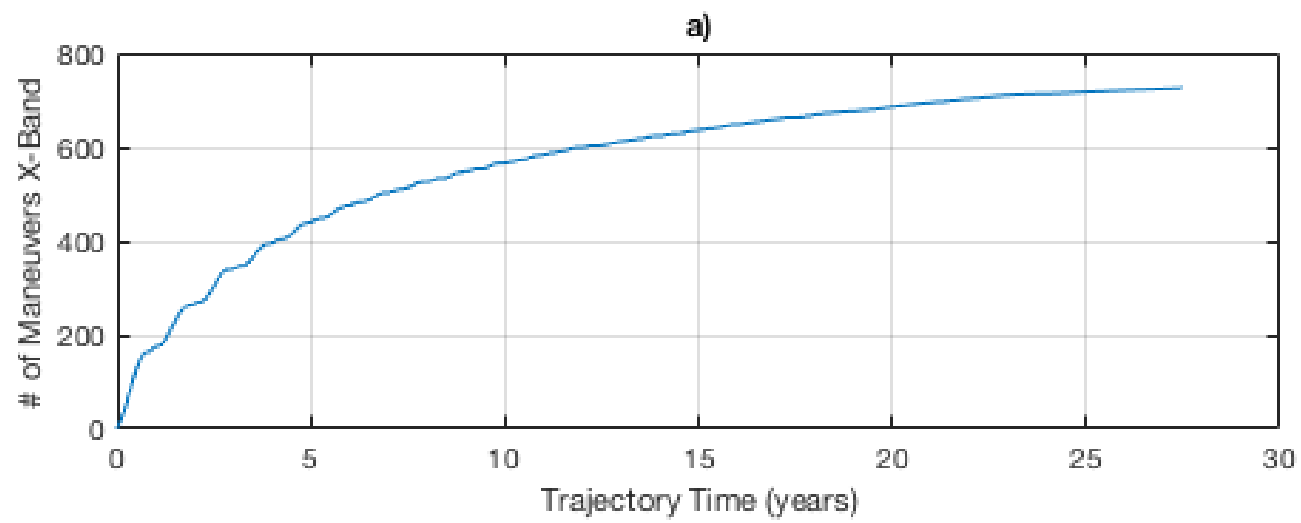

b)

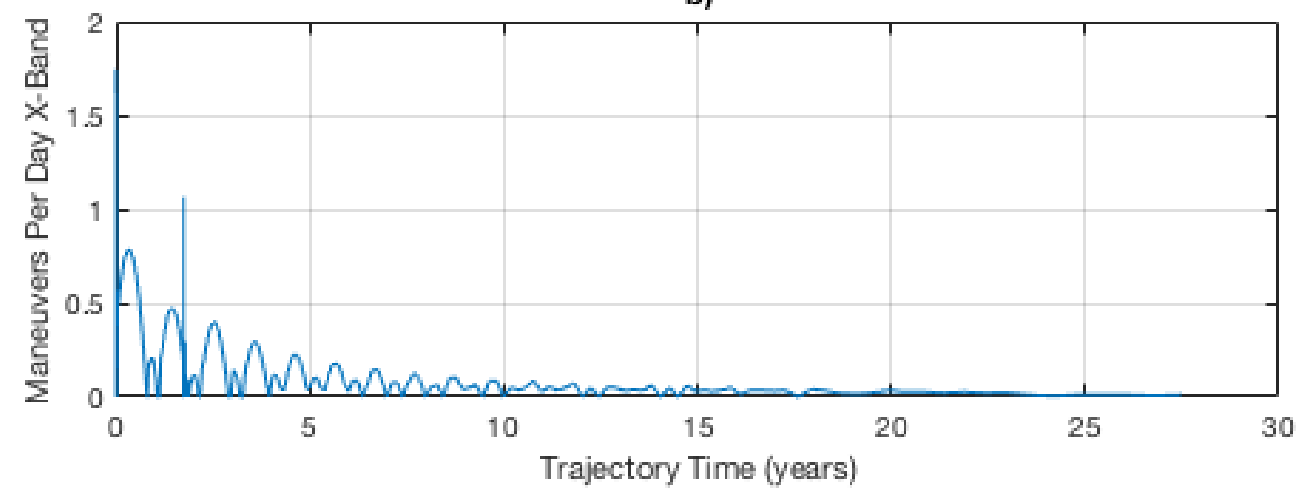

Figure 9.10: a) Number of Required Attitude Correction Maneuvers Using X-Band Beamwidth; b) Frequency of Maneuvers

As expected when using S-Band, the larger beamwidth requires the spacecraft to perform an attitude change less frequently, resulting in a lower total number of attitude maneuvers. However, X-Band will be necessary at distances of 100 AU to close the link with reasonable electrical power. When closer to the Sun, the spacecraft can utilize S-Band to minimize the amount of maneuvers to change the spin axis attitude, thus reducing the number of times the catalyst bed must be heating and the average power. Once the required RF power becomes too large to close the communication link with S-Band, the spacecraft can switch to X-Band to reduce the necessary electrical power. Nonetheless, the frequency of maneuvers when using X-Band at 100 AU drops to approximately one maneuver every 100 days. 


\subsection{Pointing Budget}

The spacecraft will need to be able to accurately point towards the Earth when at large distances away. The pointing requirement for the spacecraft will be dictated by the beamwidth of the high gain antenna at X-Band. The beamwidth of a parabolic dish, $\theta_{B W}$, can be approximated by Equation 9.6, where $\lambda$ is the wavelength of the $\mathrm{RF}$ frequency and $D$ is the diameter of the dish.

$$
\theta_{B W}=\frac{70 \lambda}{D}
$$

Using a dish diameter of $7.75 \mathrm{~m}$, the beamwidths for S-Band and X-Band are 1.23 and 0.322 degrees, respectively. Because the spacecraft will use X-Band at $100 \mathrm{AU}$, the pointing accuracy requirement for the spacecraft will be 0.322 degrees.

The attitude determination and control system will need to control the pointing of the spacecraft to 0.322 degrees. There are three main factors that influence this type of spacecraft's ability to maintain this pointing rate: 1) attitude sensor accuracies, 2) spin stabilization accuracies, and 3) minimum impulse bit of the thruster.

The newest generation of nano-satellite sun sensors are seeing improved accuracies, while maintaining low power consumptions. NewSpace Systems produces the NFSS411 sun sensor that boasts accuracies of less than 0.1 degrees, while consuming 37.5 $\mathrm{mW}$ on average[28]. The sun sensor used on this spacecraft will be based off the NFSS-411. Although dependent on spacecraft geometry, spin rate, and perturbation environment, spin stabilized spacecraft can maintain inertial pointing accuracy up to 0.1 degrees[53]. Finally, the minimum impulse bit of the thruster must be small enough to allow for accurate maneuvering of the spin axis. The angle change as a function of spin rate and moment arm using Equation 9.1. The results can be seen in Figure 9.11. These results assume the BGT-X1 minimum impulse bit of $14 \mathrm{mNs}[37]$. 


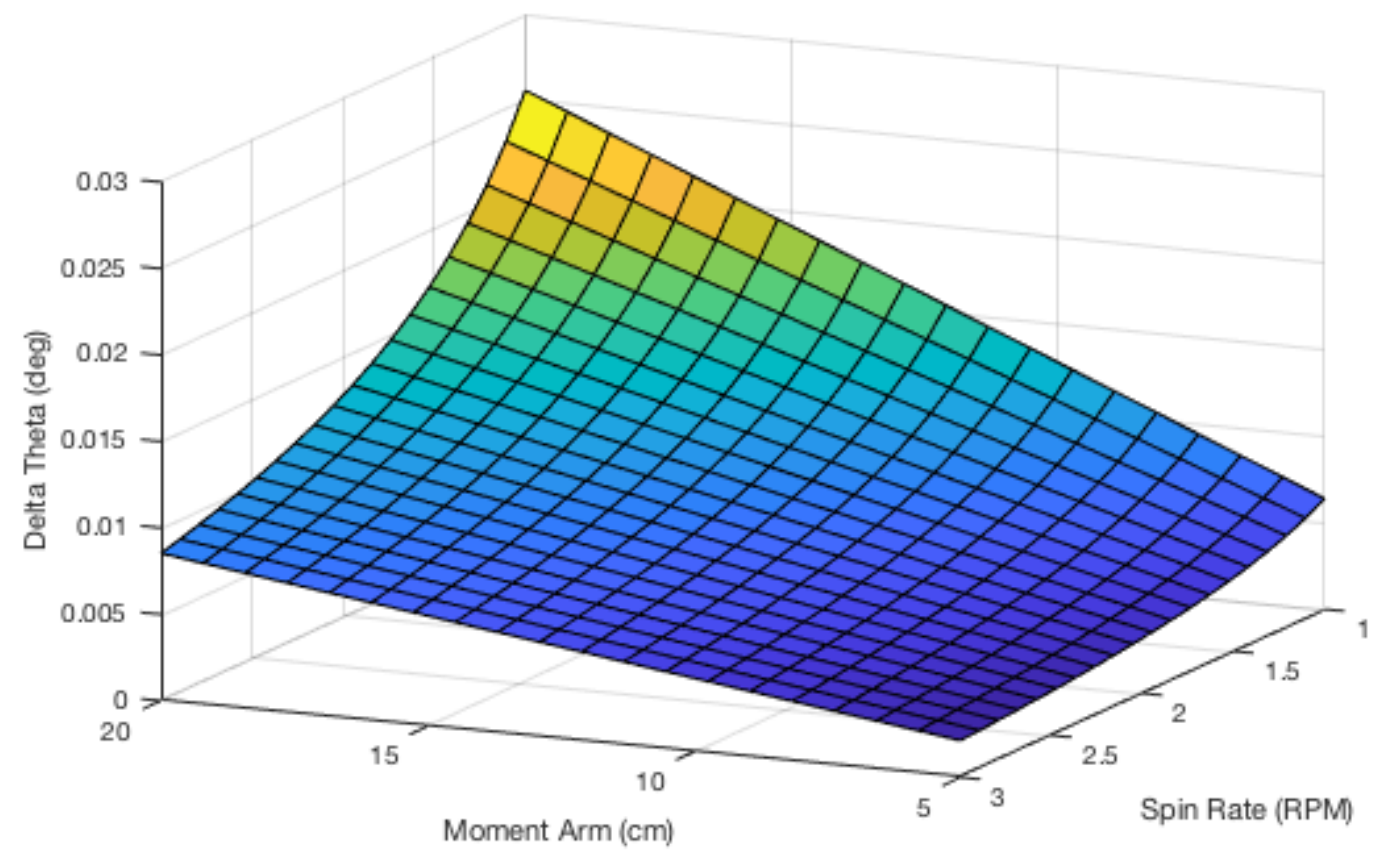

Figure 9.11: Spin Axis Angle Change for $14 \mathrm{mNs}$ Minimum Impulse Bit as a Function of Moment Arm and Spin Rate

Using the thruster configuration detailed in Figure 9.8 and a spin rate of 2 RPM, the spin axis would only shift $1.45 \mathrm{E}-4$ degrees. These values can be placed into a simple pointing budget to detail that the pointing requirement is met. The pointing budget is shown in Table 9.7 .

Table 9.7: Spacecraft Pointing Budget

\begin{tabular}{|c|c|c|}
\hline Pointing Budget Component & Value & Notes \\
\hline Pointing Requirement & $0.322 \mathrm{deg}$ & Derived from X-Band Beamwidth \\
\hline Sun Sensor Accuracy & $<0.1 \mathrm{deg}$ & Based off NFSS-411 Sun Sensor \\
Spin Stabilization Accuracy & $0.1 \mathrm{deg}$ & From SME: The New SMAD \\
Minimum Impulse Bit Accuracy & $1.45 \mathrm{E}-4 \mathrm{deg}$ & BGT-X1 Thruster \\
\hline Total Accuracy & $\mathbf{0 . 2 0 0 1} \mathbf{d e g}$ & \\
\hline Margin & $\mathbf{0 . 1 2 1 9} \mathbf{~ d e g}$ & \\
\hline
\end{tabular}


Although the pointing budget could be more in depth, including implementation accuracies and pointing accuracies of the parabolic dish, Table 9.7 details the major contributors to the pointing requirements and accuracies. Due to the tight pointing requirement, this area of the spacecraft should be looked into further.

\subsection{ADCS and Propulsion Subsystem Summary}

The ADCS and propulsion subsystem is summarized in this section. Thrusters were quickly identified as one of the few solutions to the spacecraft's attitude control and propulsion needs. Furthermore, it was determined that sun sensors and spin stabilization provide adequate accuracy for the pointing requirement, which consisted of the beamwidth of the antenna at X-Band. Table 9.8 shows the major components and values of the ADCS and propulsion subsystems.

Table 9.8: Summary of ADCS and Propulsion Subsystem Characteristics

\begin{tabular}{|c|c|}
\hline Pointing Requirement & $0.322 \mathrm{deg}$ \\
\hline Total Pointing Accuracy & $0.2001 \mathrm{deg}$ \\
\hline Pointing Accuracy Margin & $0.1219 \mathrm{deg}$ \\
\hline Attitude Sensor & NewSpace Systems NFSS-411 Sun Sensor \\
\hline Spin Stabilization Spin Rate & 2 RPM \\
\hline Total Impulse Requirement & $880 \mathrm{Ns}$ \\
\hline Thruster System Total Impulse & $1100 \mathrm{Ns}$ \\
\hline Total Impulse Margin & $25 \%$ \\
\hline Attitude Actuator & Busek BGT-X1 (2 Tanks, 4 Thrusters $)$ \\
\hline
\end{tabular}

While several aspects of this subsystem should be investigated further, this provides a general idea of what the requirements and possible solutions to the ADCS and propulsion subsystem. 
Chapter 10

\section{SYSTEM CONFIGURATION AND CONFIGURATION OPTIONS}

The configuration of the spacecraft is quite unique due to its unique mission. However, this configuration is not the only possibility. As mentioned in the Deployable Subsystem chapter, Babuscia's inflatable antenna was selected due to its scalability, low mass, and compact qualities. While these parameters were ideal for the spacecraft, the inflatable antenna is sub-optimal when placing the payload. The magnetometer can be placed anywhere on the spacecraft to obtain measurements, however, the plasma instrument must be placed clear of the dish, as the dish creates a wake for these plasma particles and will compromise the measurements. In this chapter, the configuration of the spacecraft using Alessandra Babuscia's bubble will be explained, the mass budget will be detailed, and alternative configuration options will be presented.

\subsection{Spacecraft Configuration}

This section details the configuration of the various spacecraft components. These components can be split up into three main categories, the deployable antenna, the payload, and the bus.

\subsubsection{Deployable Antenna Configuration}

The deployable antenna will be housed in a $33 \mathrm{~cm} \mathrm{x} 33 \mathrm{~cm}$ x $15 \mathrm{~cm}$ compartment that doubles as the deployable radiator system. Because the solar cells must be able to rotate 180 degrees, the solar cells must sit slightly above the radiator face. If the solar cell width is assumed to be $5 \mathrm{~cm}$, this requires the solar cells to maintain a gap of at least $2.5 \mathrm{~cm}$ from the bottom surface. The louvers will sit $3 \mathrm{~cm}$ above the surface 
to guarantee the ability to rotate. This leave the inflatable antenna $12 \mathrm{~cm}$ of height in the compartment. This $33 \mathrm{~cm} \times 33 \mathrm{~cm} \times 13 \mathrm{~cm}$ volume equates to just over $13 \mathrm{U}$ of volume, satisfying the volume requirements for this dish size. The antenna will sit above the solar cells as shown in Figure 10.1.

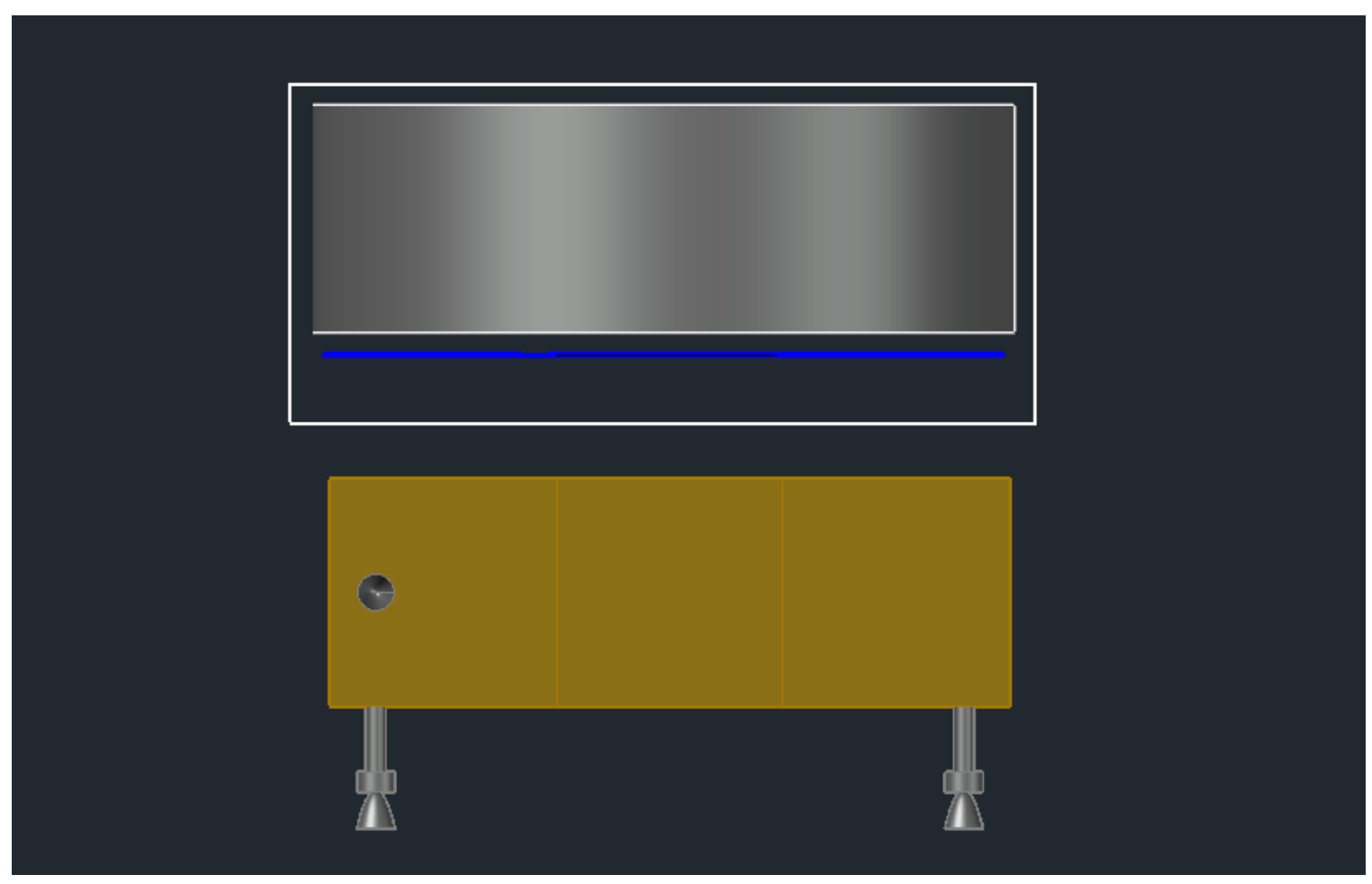

Figure 10.1: Inflatable Antenna Configuration When Packaged

While the packaged antenna is a relatively simple problem to solve, a more difficult challenge will be that of attaching the inflatable antenna to the face. This configuration issue should be investigated further with better knowledge of the inflatable antenna system itself.

\subsubsection{Payload Configuration}

The most important aspect of the spacecraft is the payload, as the scientific measurements from these instruments motivate the design for this spacecraft. However, there 
is an inherent problem with using an inflatable dish. While the magnetometer can be placed anywhere on the spacecraft, the plasma instrument must be able to detect the space environment unperturbed by the large dish. The spacecraft's large dish will interfere with the natural plasma environment as it travels to the outer solar system. Ideally the payload could be placed on the front of the spacecraft to measure this unperturbed space, however, Babuscia's inflatable dish does not have many structurally rigid components. Therefore, the payload must be placed on booms to clear the edge of the dish. Fortunately, state-of-the-art boom technology produces very low mass booms. According to the Planetary Society's online resources for Lightsail, the four booms, each 4 meters in length, deployed out of a $0.5 \mathrm{U}$ compartment[72]. Furthermore, a study of the possible boom materials for the NEAScout spacecraft showed that an alternative material option of carbon fiber and epoxy booms had linear densities of 16.5 grams per meter[38]. Therefore, it is reasonable to assume that the spacecraft can use a slightly more massive variation of the booms seen on NEAScout that allows for electrical power transmission to the payload instruments. A conservative estimate of $100 \mathrm{~g} / \mathrm{m}$ linear density will be assumed for the payload booms, and these will each be housed in $0.25 \mathrm{U}$ compartments. As can be seen in Figure 4.5, the instruments are placed on the opposite end of the spin up thrusters to minimize the risk of plume impingement.

\subsubsection{Bus Configuration}

The bus contains the necessary electronics needed to make the system work. These electronics include the battery, PDU, processor, transmitter, sun sensor, and the thruster system. These electronics cover the basics of what is necessary for a working spacecraft. The components and their volumes are shown in Table 10.1.

Many of these electronics are based off of currently available COTS components 
Table 10.1: Required Bus Electronics and Dimensions

\begin{tabular}{|c|c|c|}
\hline Component & Dimensions & Reference \\
\hline Sun Sensor & $3.4 \times 3.2 \times 2.0 \mathrm{~cm}$ & NewSpace Systems NFSS-411[28] \\
Battery & $9.4 \times 8.4 \times 4.0 \mathrm{~cm}$ & Gomspace BPX[57] \\
PDU & $9.6 \times 9.0 \times 1.3 \mathrm{~cm}$ & Crystalspace P1U Vasik[59] \\
Processor & $9.0 \times 9.6 \times 1.0 \mathrm{~cm}$ & CubeSpace Cube Computer[27] \\
Transmitter & $1 \mathrm{U}$ & SME: The New SMAD [53] \\
Thruster System & $1 \mathrm{U}(2 \mathrm{x})$ & Busek BGT-X1[37] \\
\hline
\end{tabular}

with the exception of the transmitter. However this transmitter is based off of current solid state power amplifier technology, which ranges from $0.5 \mathrm{~kg}$ to $1.5 \mathrm{~kg}[53]$. The volume is estimated from the mass. The configuration of the components are shown in Figure 10.2.

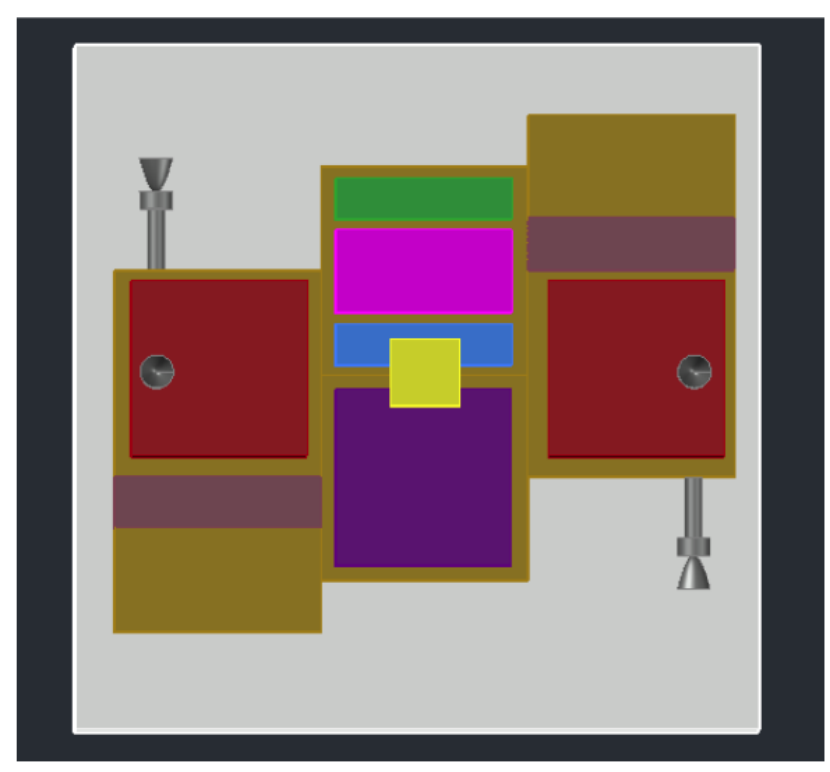

\begin{tabular}{|ll|}
\hline & \multicolumn{1}{c|}{ LEGEND } \\
Red: & Thruster Tanks \\
Purple: & SSPA Transmitter \\
Blue: & PDU \\
Pink: & Battery \\
Green: & Processor \\
Gold: & MLI \\
\hline
\end{tabular}

Figure 10.2: Component Configuration of Bus

The configuration of the components results from the spacecraft needs. The 
thruster systems were placed at the farthest end of the bus in order maximize the moment arm of the thruster. The attitude sensor is placed at the center of the bottom of the bus, as it will need to point towards the sun. The remaining electronics are packed into a $2 \mathrm{U}$ compartment to address their volume requirements. There are many different ways to configure the components however, this configuration shows a possible way to place the components.

\subsection{Mass Budget}

As state in the System Requirements Chapter, a goal for the design of the spacecraft is to keep the mass below $30 \mathrm{~kg}$. While this is not a strict requirement, keeping the spacecraft mass low helps maximize rideshare opportunities as a secondary payload. Now that the required components for all subsystems have been determined to an extent, all of the spacecraft components can be placed into a mass budget to determine the estimated mass of the system as a whole. This mass budget is displayed in Table 10.2.

Table 10.2 shows all the spacecraft components, their dimensions and masses, and the reference to current systems. $25 \%$ contingency was added to the system in order to account for inaccuracies in the mass estimation. With the added $25 \%$ contingency, the spacecraft turns out to be just under $31 \mathrm{~kg}$, very close to the goal originally set at the start of the paper. This total mass is rather impressive for a spacecraft that can operate from 5 to 100 AU without the use of an RTG power source. This will help maximize the possibilities of employing a rideshare system with a host spacecraft or hosting multiple of these spacecraft for use in a mission using a swarm architecture. 


\section{Table 10.2: Spacecraft Master Mass Budget}

\begin{tabular}{|c|c|c|c|}
\hline Component & Dimensions & Mass (kg) & Reference \\
\hline \multicolumn{4}{|c|}{ Bus } \\
\hline Inflatable Antenna & $13 \mathrm{U}$ & 13.000 & Babuscia Inflatable Antenna[3] \\
\hline Sun Sensor & $3.4 \times 3.2 \times 2.0 \mathrm{~cm}$ & 0.035 & NewSpace Systems NFSS-411[28] \\
\hline Battery & $9.4 \times 8.4 \times 4.0 \mathrm{~cm}$ & 0.500 & Gomspace BPX[57] \\
\hline PDU & $9.6 \times 9.0 \times 1.3 \mathrm{~cm}$ & 0.100 & Crystalspace P1U Vasik[59] \\
\hline Processor & $9.0 \times 9.6 \times 1.0 \mathrm{~cm}$ & 0.060 & CubeSpace Cube Computer[27] \\
\hline Transmitter & $1 \mathrm{U}$ & 1.000 & SME: The New SMAD[53] \\
\hline Thruster System & $1 \mathrm{U}(2 \mathrm{x})$ & 3.000 & Busek BGT-X1[37] \\
\hline Solar Cell Louvers & $0.1089 \mathrm{~m}^{2}$ & 0.540 & $5 \mathrm{~kg} / \mathrm{m}^{2}$ Areal Density[53] \\
\hline Bus Structure & $\approx 6 \mathrm{U}$ & 1.000 & ISIS 6U Cubesat Structure[73] \\
\hline MLI & $0.22 \mathrm{~m}^{2}$ & 0.360 & Areal Density: $1.5 \mathrm{~kg} / \mathrm{m}^{2}[64]$ \\
\hline Deployable Radiators & $0.3069 \mathrm{~m}^{2}$ & 2.450 & Areal Density: 8 kg/m²[65] \\
\hline \multicolumn{4}{|c|}{ Payload } \\
\hline Magnetometer & $0.5 \mathrm{U}$ & 0.500 & INSPIRE Magnetometer[22] \\
\hline Plasma Instrument & $0.5 \mathrm{U}$ & 0.500 & Similar to Magnetometer \\
\hline Booms $(2 \mathrm{x})$ & $8.25 \mathrm{~m}$ Total & 1.000 & Linear Density 0.1 kg/m[38] \\
\hline Boom Deploymers (2x) & $0.5 \mathrm{U}$ Total & 0.500 & Lightsail[38] \\
\hline Total Mass $+25 \%$ Contingency & \multicolumn{3}{|c|}{30.610} \\
\hline
\end{tabular}

\subsection{Alternative Configuration Options}

While this spacecraft represents just one of the ways that can satisfy mission requirements, there are countless ways to configure a spacecraft. Particularly for this spacecraft, the deployable system dictates many configuration decisions, such as payload placement. An alternative deployment system could result in a drastically different system. Many boom deployed systems are under development, while some are commercially available now. For example, Tendeg's product, the KaTENna High Gain Cubesat Antenna, is a boom deployed system that uses tensioners to produce the parabolic dish shape. A rendering of the KaTENna shown on Tendeg's website is 
displayed in Figure 10.3.

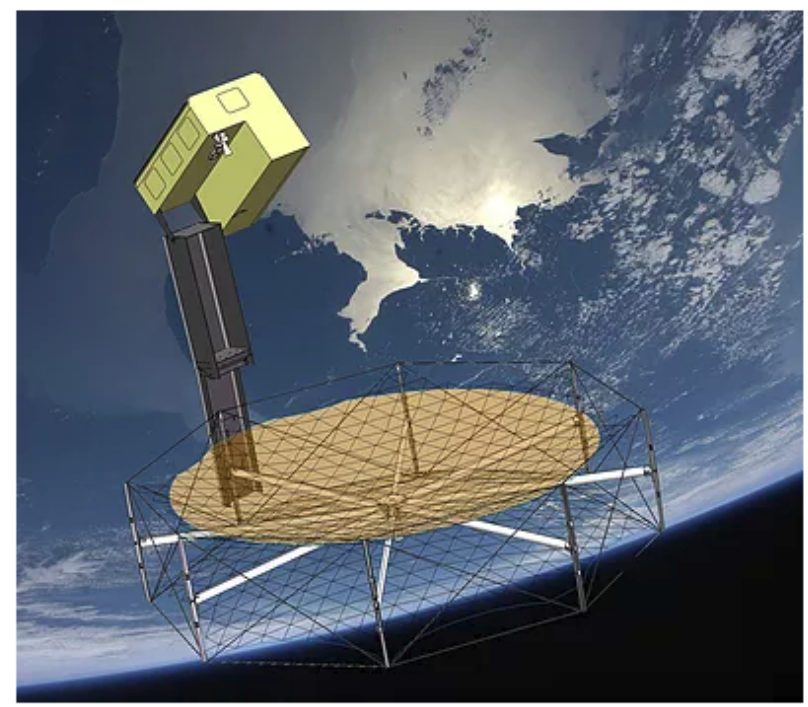

Figure 10.3: Tendeg's Off-Center Fed KaTENna High Gain Cubesat Antenna[12]

With this boom deployed parabolic dish, it would be relatively simple to place the payload on the outside of this dish, in order to accurately take scientific measurements of deep space without the need for payload booms. A simple visualization of this can be seen in Figure 10.4. 


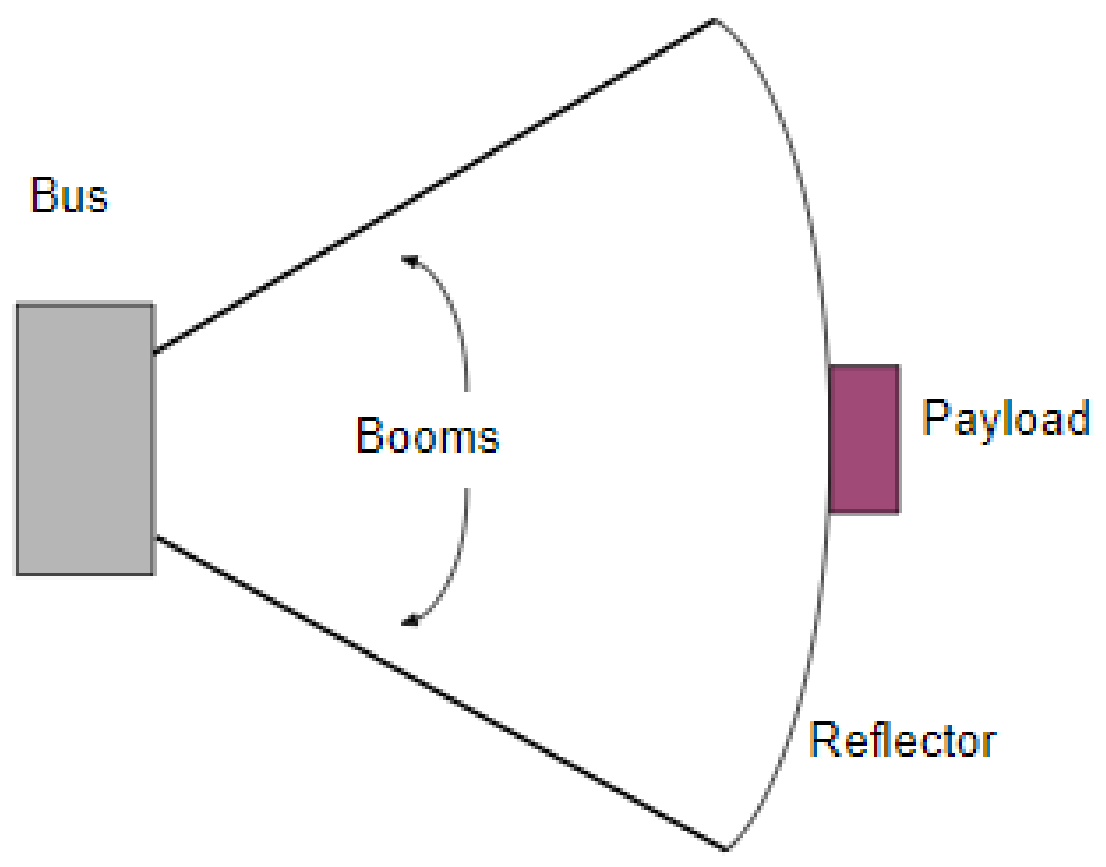

Figure 10.4: Alternative Configuration Using Boom Deployed Parabolic Dish

While this configuration appears much more practical than the combination of the inflatable antenna and payload booms, it should be kept in mind that current boom deployed reflectarray antennas have high masses and volumes. Tendeg advertises its nominal KaTENna configuration of a $1 \mathrm{~m}$ effective aperture. This system has a mass of $2.5 \mathrm{~kg}$ and a $3 \mathrm{U}$ volume, $500 \%$ more massive and $600 \%$ more volume than the inflatable antenna. However, as deployable technology improves and packing efficiencies increase, these deployable systems should be revisited to consider the boom deployed configuration for the spacecraft. 


\section{Chapter 11}

\section{RADIATION CONSIDERATIONS}

While radiation is not observed in depth for the design spacecraft, it is worth stating the radiation considerations for this type of mission. The majority of the spacecraft life will be spent in interplanetary space as it escapes the solar system. The radiation in this region of space consists mostly of galactic cosmic rays (GCRs). Although the particles come far and few between, the particles are very high energy and difficult to shield. For a long voyage to the outer solar system, the total ionization dose (TID) could build up to high levels.

While the GCRs in interplanetary space can be harmful to the spacecraft's electronics, interplanetary space is relatively benign compared to other space environments. A paper by H.B. Garrett, A.A. Shapiro, and J.Y. Yang detail the radiation environment in various regions of the solar system. This information can be seen in Figure 11.1. 


\begin{tabular}{|c|c|c|c|c|c|c|c|c|}
\hline \multirow[b]{2}{*}{ Mission } & \multirow[b]{2}{*}{$\begin{array}{c}\text { Dominant } \\
\text { Species }\end{array}$} & \multicolumn{2}{|c|}{ Total Dose (krad//vr) } & \multirow{2}{*}{$\begin{array}{c}\text { Total } \\
\text { SEU Risk } \\
\text { (Relative) }\end{array}$} & \multicolumn{4}{|c|}{ SEU Susceptibility (bit-flip/Gbits/yr) [1] } \\
\hline & & $\begin{array}{c}\text { With } \\
100 \mathrm{mil} \text { Al }\end{array}$ & $\begin{array}{c}\text { With } \\
400 \text { mil Al }\end{array}$ & & $\begin{array}{l}\text { Trapped } \\
\text { Protons } \\
\end{array}$ & $\begin{array}{l}\text { Cosmic } \\
\text { Ravs [2] }\end{array}$ & $\underline{\text { Solar } \mathrm{Pr}}$ & $\frac{\text { tons [3] }}{400 \mathrm{mil} \mathrm{Al}}$ \\
\hline LEO & $\mathrm{p}+$ & $<1$ & $<<1$ & Low & + & $10^{-2}$ & 0 & 0 \\
\hline LEO-Polar & $p+$ & 6 & 1 & Moderate & + & $10^{-2}$ & $10^{-2}$ & $10^{-5}$ \\
\hline MEO & $\theta-($ soft $)$ & $200-2000$ & $10-100$ & Severe & ++ & $10^{-2}$ & $10^{-3}$ & $10^{-4}$ \\
\hline GEO & $e-$ (soft) & $<100$ & $<1$ & Moderate & 0 & $10^{-2}$ & $10^{-3}$ & $10^{-4}$ \\
\hline Jup-Galileo & e-(hard) & 250 & 25 & Severe & ++ & $10^{-2}$ & 0 & 0 \\
\hline Jup/Europa & e - (hard) & 1500 & 800 & Severe & ++ & $10^{-2}$ & 0 & 0 \\
\hline Interplanetary & $p+$ & $<1$ & $<<1$ & Low & 0 & $10^{-2}$ & $10^{-1}$ & $10^{-3}$ \\
\hline $\mathrm{DOD} /$ Nuclear & $e-($ suft $)$ & $50-10000$ & $1-100$ & Severe & $\mathrm{NA}$ & $\mathrm{NA}$ & $\mathrm{NA}$ & $\mathrm{NA}$ \\
\hline
\end{tabular}

Notes:

1 For devices with LET $=10 \mathrm{MeV}-\mathrm{cm}^{2} / \mathrm{mg}$

2 For Adams' 90\% Worst Case GCR (Galactic Cosmic Rays)

3 For 99th percentile Solar Flare Events

Figure 11.1: Radiation Environments in Various Regions of the Solar System[13]

Observing the information detailed in Figure 11.1, radiation in interplanetary space is much less dangerous than orbiting Earth or Jupiter, with the exception of in Low Earth Orbit (LEO). With 100 mil of aluminum shielding, the radiation dosage can be minimized to less than 1 krad per year. Over the 30 year voyage to $100 \mathrm{AU}$, the TID for the spacecraft with 100 mil of aluminum shielding could be reduced to tens of krads. Current COTS CubeSat components already advertise radiation tolerant systems capable of receiving a TID of tens of krads. For example, the processor used in this spacecraft is based off of the CubeComputer, a processor developed by CubeSpace. The CubeComputer is advertised to handle $20 \mathrm{krad}$ of TID and is protected against Single Event Upsets (SEUs) and Single Event Latchups (SELs) through advanced software techniques. However, 100 mil of shielding is relatively thick, corresponding to $2.54 \mathrm{~cm}$ thick aluminum. If placed around the entire $6 \mathrm{U}$ bus and assuming a density of aluminum of $2700 \mathrm{~kg} / \mathrm{m}^{3}$, the resulting mass of the shielding would be roughly $15 \mathrm{~kg}$. On the other hand, if this same shielding was 
applied to a $2 \mathrm{U}$ volume containing the sensitive electronics, the resulting mass of the shielding would be just under $7 \mathrm{~kg}$.

However, the spacecraft will need to survive more than just the interplanetary radiation. As mentioned in the Orbital Trajectory Options chapter, reaching solar system escape velocity will likely require a flyby of either Jupiter or Saturn. Although Saturn's radiation belts are much weaker than those around Jupiter, the spacecraft would receive significant amounts of radiation, especially with low periapse flyby maneuvers. There are other orbital solutions to reach solar system escape velocities, such as those studied by NASA and KISS that utilize low perihelion maneuvers, however, the spacecraft will need to make use of a gravitational assist maneuver in order to reach a different $100 \mathrm{AU}$ destination than the primary spacecraft and return useful scientific data. A mitigation strategy to reduce the radiation exposure to the electronics would be to shut of the spacecraft as it travels through the harmful radiation belts. Without the spacecraft running, the electronics will receive less radiation damage as a result.

It is reasonable to assume that in the near-future, nano-satellite electronics will become more radiation tolerant, requiring less shielding. The radiation environment and available radiation tolerant components for this type of mission should be explored in more detail as further improvements in nano-satellite electronics are developed. 
Chapter 12

\section{CONCLUSION}

Overall, this paper demonstrates that the concept of a solar powered deep space small satellite can work using relatively low-cost and lightweight components. While the paper details one specific satellite design, it is important to note that there can be many other configurations that can satisfy the deep space mission requirements. With new advanced nano-satellite electronics constantly reducing in size and power consumption coupled with advancements in small spacecraft deployable systems, the task of generating sufficient electrical power will only become easier. While the spacecraft presented in this paper may not be the ideal design for the mission, it lays out the necessary requirements and possible solutions to satisfy these requirements. Stemming from these requirements, multiple bus configurations, deployable antenna systems, payload selections and other trades not included in the scope of this paper may be examined in the future to determine the truly ideal design choices for the solar powered deep space small spacecraft.

While this paper covers a large breadth of topics concerning the requirements and spacecraft design options for this type of spacecraft, most all of the topics can be investigated further to determine the true intricacies of designing a functional solar powered deep space probe. The probe design put forward in this paper is the result of relatively light analysis and many assumptions. However, the simplified bulk analysis shows that this type of system can operate under reasonable assumptions.

All in all, the analysis in this paper shows promise that a design for a solar powered deep space small spacecraft can function and return sufficient and useful data using low-cost and lightweight components. While this paper acts as a stepping stone for this type of spacecraft design, laying out fundamental requirements and 
possible solutions, future work involves examining the various aspects of this mission in further detail to truly determine the best design solutions.

\subsection{Further Work}

There are several key aspects that warrant some additional more detailed analysis in order to verify some of the bulk analysis and assumptions of this paper.

The deployable antenna is easily the most unique and important feature of the spacecraft, serving a dual purpose of concentrating sunlight and operating as a high gain antenna. While many deployable systems are in development, almost all are exclusively used as antennas. The second purpose of the deployable dish of concentrating sunlight should be looked into further. Advanced optical analysis software should be used to determine the true effectiveness of this multi-purpose reflector. This multi-purpose dish should ideally be developed specifically for this type of mission and tested to determine deployment options and antenna and solar concentrator efficiencies. The proposed spacecraft used an inflatable antenna dish, however, boom deployed systems should be investigated. These boom deployed systems are inherent more complicated and likely more massive than the inflatable bubble, however, as lightweight boom technology develops, these systems should become more lightweight and able to pack into smaller compartments. The boom deployed system offers the advantage of structural rigidity for the parabolic reflector. This would allow for the payload to be positioned at the front of the spacecraft, allowing the instruments to measure unperturbed deep space. Tensioners should be able to control and maintain the necessary shape of the dish. While these deployable systems are inherently

complicated, options should be reviewed as technological advancements in this area progresses.

Additional thermal and structural analysis should be performed on the spacecraft. 
This paper lacks the analysis of the structural subsystem in particular. While the CubeSat form factor has been proven to be structurally sufficient, this unique design warrants more in depth investigation. The structural subsystem also plays a key role in keeping the spacecraft thermally stable and within operating temperature limits, especially for the bus. A critical aspect of keeping the bus within temperature limits at both extremes of the thermal environment is minimizing the conductive path between the radiator panels of the spacecraft and bus itself. Reducing the conduction through the structure attaching these two components allows the bus to maintain adequate temperatures using the thermal dissipation from components alone. This means the bus will not receive too much thermal energy when close to the sun, as well as avoid conducting necessary thermal energy to the radiators when at the cold 100 AU case. The structural connections between the bus and the radiators will need to be structurally and conductively sufficient.

The lifetime and redundancy aspect of this spacecraft should examined further to determine the redundancy requirements from a component to a system level. While the spacecraft relies on low power COTS nano-satellite electronics, these components are usually rated for a maximum lifetime of several years. Using traditional flyby trajectories, the travel time to $100 \mathrm{AU}$ takes 30 years. Some of the most robust spacecraft are designed to last a maximum of 15 years. However, as stated in a paper by Robert Staehle et al., nano-satellite components tend to operate better and longer than most anticipate. In fact, as of 2012, University of Tokyo's Cubesat XI-IV has reached 9 years of operational lifetime, after having been launch in a sun synchronous orbit in June of 2003[74]. In-the-sky.org, a satellite monitoring website, states that the spacecraft is still operational today[75]. Nonetheless, with the lengthy travel time to reach the outer solar system, redundancy requirements and radiation dosage should also be investigated to determine the rad-hard requirements for the electronics on this type of mission. 


\section{BIBLIOGRAPHY}

[1] NASA. Mars Cube One. https://www.jpl.nasa.gov/cubesat/missions/marco.php.

[2] Scienceogram. "The cost of space missions". https://scienceogram.org/blog/2015/07/space-missions-cost-new-horizons/, July 2015.

[3] Alessandra Babuscia, Thomas Choi, Charles Lee, and Kar-Ming Cheung. Inflatable antennas and arrays for interplanetary communication using CubeSats and smallsats, 2015.

[4] David Gilmore. Spacecraft Thermal Control Handbook, volume 1. The Aerospace Corporation, December 2002.

[5] Erik Gregersen. "Heliosphere - Astronomy". Encyclopedia Brittanica. https://www.britannica.com/science/heliosphere.

[6] R.A. Wallace, J.A. Ayon, and G.A. Sprague. "Interstellar Probe mission/system concept". Aerospace Conference Proceedings, 2000 IEEE, 7:385-396, March 2000. https://ieeexplore.ieee.org/document/879304/.

[7] Randall C. Hopkins, Herbert D. Thomas, Bruce M. Weigmann, Andrew F. Heaton, Les Johnson, Michael F. Baysinger, and Benjamin R. Beers. "Propulsion Technology Assessment: Science Enabling Technologies to Explore the Interstellar Medium". NASA Technical Reports Server, 2016.

[8] Alessandra Babuscia, Thomas Choi, Kar-Ming Cheung, Aman Chandra, and Jekan Thangavelautham. Inflatable antenna for CubeSats: Development of the X-Band, March 2016. 
[9] Paul Wade. Parabolic Dish Focus, Zoom, and Tilt. http://educypedia.karadimov.info/library/Parabolic_Dish_Focus_Zoom_and_Tilt.pdf, 2009.

[10] Howard Haber. Focusing properties of spherical and parabolic mirrors. UCSanta Cruz Physics, 2009. http://scipp.ucsc.edu/ haber/ph5B/parabolic09.pdf.

[11] Small Solar Power Sail Demonstrator 'IKAROS' Successful Attitude Control by Liquid Crystal Device.

http://global.jaxa.jp/press/2010/07/20100723_ikaros_e.html, July 2010. Press Release.

[12] KaTENna High Gain Cubesat Antenna. Tendeg Space Antennas and Deployables. https://www.tendeg.com/products.

[13] H.B. Garrett, A.A. Shapiro, and J.Y. Yang. Interstellar Space Missions: Ultra Reliability and Engineering Issues - Part ii. 46th AIAA Aerospace Sciences Meeting and Exhibit, January 2008.

[14] Ralph McNutt. "Near-Term Exploration of the Interstellar Medium". 68th International Astronautical Congress (IAC) Adelaide, Australia, 2018.

[15] NASA. "The Pioneer Missions", March 2007.

[16] NASA-JPL. "Solar System Dynamics HORIZONS Web-Interface". https://ssd.jpl.nasa.gov/horizons.cgi.

[17] NASA. "Voyager Mission Status".

[18] Barrie W. Jones. "Pluto: Sentinel of the Outer Solar System". 2010.

[19] Allen Cummings and Sten Odenwald. "Issue 62: Where is the Heliopause?". NASA Sun-Earth Day: 2008 - Space Weather Around the World. https://sunearthday.nasa.gov/2008/TTT/62_heliopause.php. 
[20] Larry Klaes. "Our SpaceFlight Heritage: Project Orion, a nuclear bomb and rocket all in one". Spaceflight Insider, August 2016.

http://www.spaceflightinsider.com/space-flight-history/project-orion-nuclearbomb-rocket/.

[21] Edward Stone, Leon Alkalai, and Louis Friedman. "Science and Enabling Technologies for the Exploration of the Interstellar Medium". Keck Institute for Space Studies, 2015. http://www.deepspace.ucsb.edu/wpcontent/uploads/2015/04/Final-KISS-ISM-Report.pdf.

[22] NASA-JPL. Interplanetary Nano-Spacecraft Pathfind in Relevant Environment (INSPIRE). https://www.jpl.nasa.gov/cubesat/missions/inspire.php.

[23] NASA-JPL. Lunar Flashlight. "https://www.jpl.nasa.gov/cubesat/missions/lunar_flashlight.php".

[24] Les Johnson, Julie Castillo-Rogez, and Jared Dervan. Near-Earth Asteroid Scout: NASA's Solar Sail Mission to a NEA. 2017. https://ntrs.nasa.gov/archive/nasa/casi.ntrs.nasa.gov/20170000096.pdf.

[25] B.A. Cohen, R.G. Sellar, R. Staehle, N. Toomarian, and D.A. Paige. LUNAR FLASHLIGHT: MAPPING LUNAR SURFACE VOLATILES USING A CUBESAT. Annual Meeting of the Lunar Exploration Analysis Group (2013), 2013. https://sservi.nasa.gov/wp-content/uploads/2014/04/7045.pdf.

[26] LUNAR H-MAP SPACECRAFT. http://lunahmap.asu.edu/. Web. Accessed 3-12-2018.

[27] CubeSpace CubeComputer. CubeSatShop.com, August 2016. https://www.cubesatshop.com/wp-content/uploads/2016/06/CubeComputerBrochure-August-2016.pdf. 
[28] NewSpace Systems NFSS-411 Sun Sensor. CubeSatShop.com.

https://www.cubesatshop.com/wp-content/uploads/2016/06/NewSpace-SunSensor_6a-1.pdf.

[29] ISIS Communications Systems. https://www.cubesatshop.com/wpcontent/uploads/2016/06/Communication-systems-Brochure-v1.pdf. Brochure.

[30] James Webb Space Telescope. Northrop Grumman Corporation. http://www.northropgrumman.com/Capabilities/JWST/Pages/default.aspx.

[31] IKAROS Small Scale Solar Powered Sail Demonstration Satellite. Japan Aerospace Exploration Agency. http://www.isas.jaxa.jp/en/missions/spacecraft/current/ikaros.html.

[32] Lightsail Overview Drawing. https://planetary.s3.amazonaws.com/projects/lightsail/models/lightsailoverview-dec2016.PDF. Schematic.

[33] Juno Mission to Jupiter. https://www.jpl.nasa.gov/news/fact_sheets/JUNO_Fact_Sheet_2016.pdf, September 2015. JUNO Fact Sheet.

[34] Dustin McNulty. Reflectivity Measurements. https://www2.cose.isu.edu/ mcnudust/publication/presentations/MOLLERCollabMeeting_2016Apr30_refTalk.pdf, April 2016.

[35] Timothy Pham. Deep Space Network Services Catalog. https://deepspace.jpl.nasa.gov/files/820-100-F1.pdf. 
[36] The "Dish" Radio Antenna Facility. https://www.sri.com/researchdevelopment/specialized-facilities/dish-radio-antenna-facility.

[37] Busek Space Propulsion and Systems - Green Monopropellant Thrusters. http://www.busek.com/technologies__greenmonoprop.htm.

[38] Juan Fernandez, Geoffrey Rose, Casey Younger, Greg Dean, Olive Stohlman, Jay Warren, and W. Keats Wilkie. NASA's Advanced Composites-based Solar Sail System for Interplanetary Small Satellite Missions. DEEP SPACE COMMUNICATIONS AND NAVIGATION SERIES, January 2017.

[39] Nick Anthony Fiorenza. Synodic Cycles and Planetary Retrogrades. https://www.lunarplanner.com/HCpages/SynodicCycle.html.

[40] NASA-JPL. Radar in a CubeSat (RainCube). https://www.jpl.nasa.gov/cubesat/missions/raincube.php.

[41] J. Sauder, N. Chahat, and M. Thomson. Ultra-Compact Ka-Band Parabolic Deployable Antenna for RADAR and Interplanetary Cubesats. 29th Annual AIAA/USU Conference on Small Satellites, August 2015.

[42] Charles McGillivray. Mini Deployable High Gain Antenna for CubeSats. http://mstl.atl.calpoly.edu/ bklofas/Presentations/DevelopersWorkshop2011/47_MacGillivray_Miniature_Antennas.pdf, April 2011.

[43] P.A. Warren, J.W. Steinbeck, R.J. Minelli, and C. Mueller. Large Deployable S-Band Antenna for a 6U Cubesat. Physical Sciences Inc.

[44] P-DaHGR Antenna. MMA Design LLC. http://mmadesignllc.com/products/p-dahgr-antenna/. 
[45] T-DaHGR Antenna. MMA Design LLC.

http://mmadesignllc.com/products/t-dahgr-antenna/.

[46] Re-Entry: FREEDOM Drag Sail Cubesat. http://spaceflight101.com/freedom/, February 2017.

[47] NanoSail-D, Gunter's Space Page. http://space.skyrocket.de/doc_sdat/nanosail-d.htm.

[48] dragNET De-Orbit System. MMA Design LLC. http://mmadesignllc.com/products/dragnet-de-orbit-system/.

[49] DeorbitSail AMSAT-UK. https://amsat-uk.org/satellites/telemetry/deorbitsail/.

[50] About Cubesail. University of Surrey. https://www.surrey.ac.uk/surrey-space-centre/missions/cubesail.

[51] Alessandra Babuscia, Jonathan Sauder, Aman Chandra, Jekan Thangavelautham, Lorenzo Feruglio, and Nicole Bienert. Inflatable antenna for CubeSats: A new spherical design for increased X-band gain, March 2017.

[52] The Parabolic Reflector Antenna. antenna-theory.com, 2011. http://www.antenna-theory.com/antennas/reflectors/dish.php.

[53] James Richard Wertz, David F. Everett, and Jeffrey John Puschell. Space Mission Engineering: The New SMAD. Microcosm Press, Hawthorne, 2015.

[54] A. Boca and J. Grandidier. Advanced-Architecture High Efficiency Solar Cells for Low Irradiance Low Temperature LILT Applications. 2017.

[55] Scott Benson. Solar Power for Outer Planets Study. November 2007. https://www.lpi.usra.edu/opag/nov_2007_meeting/presentations/solar_power.pdf. 
[56] Gomspace NanoPower BP4 Datasheet.

https://gomspace.com/UserFiles/Subsystems/datasheet/gs-ds-nanopower-bp426.pdf.

[57] Gomspace NanoPower BPx Datasheet.

https://gomspace.com/UserFiles/Subsystems/datasheet/gs-ds-nanopower-bpx3-18.pdf.

[58] Gomspace NanoPower BPx.

https://gomspace.com/UserFiles/Subsystems/flyer/gomspace_nanopower_bpx_flyer.pdf.

[59] Crystalspace P1U "Vasik" Power Supply. https://www.cubesatshop.com/wpcontent/uploads/2016/06/P1U-Power-Supply-summary-v1.1.pdf.

Brochure.

[60] Pat Beauchamp, Richard Ewell, Erik Brandon, and Rao Surampudi. Solar Power and Energy Storage for Planetary Missions.

https://www.lpi.usra.edu/opag/meetings/aug2015/presentations/day-

2/11_beauchamp.pdf, August

2015 .

[61] ArgoMoon Propulsion System. http://www.cubesat-propulsion.com/wpcontent/uploads/2017/08/X17025000-data-sheet-073117.pdf, July 2017.

[62] NewSpace Systems Magnetometer - NMRM-001-485.

https://www.cubesatshop.com/wp-content/uploads/2016/06/NewSpaceMagnetometer_7a-1.pdf.

Brochure.

[63] Matthew C. Deans, Steve R. Oleson, James Fittje, Anthony Colozza, Tom Packard, John Gyekenyesi, Christopher H. McLean, and Ronald A. Spores. An 
Evaluation of the Impacts of AF-M315E Propulsion Systems for Varied Mission Applications.

https://ntrs.nasa.gov/archive/nasa/casi.ntrs.nasa.gov/20150021510.pdf, 2015.

[64] Takeshi Miyakita, Ryuta Hatakenaka, Hiroyuki Sugita, Masanori Saitoh, and Tomoyuki Hirai. "Evaluation of Thermal Insulation Performance of a New Multi-Layer Insulation with Non-Interlayer-Contact Spacer" . 45th International Conference on Environmental Systems, 2015. https://ttu-ir.tdl.org/ttuir/bitstream/handle/2346/64487/ICES_2015_submission_220.pdf?sequence=1isAllowed=y.

[65] Thermally Efficient Deployable Radiators - Thermal Management Technologies. http://www.tmt-ipe.com/forms/deployableRadiatorFactSheet.pdf.

[66] William A. Imbriale. Large Antennas of the Deep Space Network. DEEP SPACE COMMUNICATIONS AND NAVIGATION SERIES, February 2002. https://descanso.jpl.nasa.gov/monograph/series4/Descanso_Mono4_web.pdf.

[67] CubeSpace - CubeWheel Reaction/Momentum Wheel. https://www.cubesatshop.com/wp-content/uploads/2016/06/CubeWheelBrochure-August-2016.pdf.

Brochure.

[68] YK. BAE CORP - Revolutionary Photonic Systems. http://ykbcorp.com/, 2017.

[69] Busek Micro Resistojet. http://www.busek.com/index_htm_files/70008518B.pdf, 2013. Brochure.

[70] Propulsion Unit for CubeSats. http://www.cubesat-propulsion.com/wpcontent/uploads/2015/10/Propulsion-unit-for-cubesats.pdf, 2014. Brochure. 
[71] BGT-X5 Green Monopropellant Thruster.

http://www.busek.com/index_htm_files/70008517E.pdf, 2017. Brochure.

[72] Lightsail Overview Drawing.

https://planetary.s3.amazonaws.com/projects/lightsail/models/lightsailoverview-dec2016.PDF. Spacecraft

Schematic.

[73] 6-Unit CubeSat structure.

https://www.isispace.nl/product/6-unit-cubesat-structure/. Brochure.

[74] Robert Staehle, Brian Anderson, Bruce Betts, Diana Blaney, Channing Chow, Louis Friedman, Hamid Hemmati, Dayton Jones, Andrew Klesh, Paulett Liewer, Joseph Lazio, Martin Wen-Yu Low, Pantazis Mouroulis, Neil Murphy, Paula J. Pingree, Jordi Puig-Suari, Tomas Svitek, Austin Williams, and Thor Wilson. Interplanetary Cubesats: Opening the Solar System to a Broad Community at a Lower Cost. December 2012.

https://www.nasa.gov/pdf/716078main_Staehle_2011_PhI_CubeSat.pdf.

[75] Cubesat XI-IV - Satellite Catalog. https://in-the-sky.org//spacecraft.php?id=27848skin=1. Satellite Catalog.

[76] NASA-JPL. "Voyager Mission - Did You Know?". https://voyager.jpl.nasa.gov/mission/did-you-know/. 